\title{
NEAR-INFRARED PHOTOMETRIC VARIABILITY OF STARS TOWARD THE ORION A MOLECULAR CLOUD
}

\author{
John M. CARPENTER AND LyNNE A. HillenBrand \\ Department of Astronomy, MS 105-24, California Institute of Technology, 1201 East California Boulevard, Pasadena, CA 91125; \\ jmc@astro.caltech.edu, lah@astro.caltech.edu \\ AND \\ M. F. SKRUTSKIE \\ Department of Astronomy, Lederle Graduate Research Tower, University of Massachusetts, Amherst, MA 01003; \\ skrutski@north.astro.umass.edu \\ Received 2001 January 3; accepted 2001 February 26
}

\begin{abstract}
We present an analysis of $J, H$, and $K_{s}$ time-series photometry obtained with the southern 2MASS telescope over a $0.84 \times 6^{\circ}$ region centered near the Trapezium region of the Orion Nebula cluster. These data are used to establish the near-infrared variability properties of pre-main-sequence stars in Orion on timescales of $\sim 1-36$ days, $\sim 2$ months, and $\sim 2$ years. A total of 1235 near-infrared variable stars are identified, $\sim 93 \%$ of which are likely associated with the Orion A molecular cloud. The variable stars exhibit a diversity of photometric behavior with time, including cyclic fluctuations with periods up to 15 days, aperiodic day-to-day fluctuations, eclipses, slow drifts in brightness over 1 month or longer, colorless variability (within the noise limits of the data), stars that become redder as they fade, and stars that become bluer as they fade. The mean peak-to-peak amplitudes of the photometric fluctuations are $\sim 0.2$ mag in each band, and $77 \%$ of the variable stars have color variations less than 0.05 mag. The more extreme stars in our sample have amplitudes as large as $\sim 2$ mag and change in color by as much as $\sim 1$ mag. The typical timescale of the photometric fluctuations is less than a few days, indicating that nearinfrared variability results primarily from short-term processes. We examine rotational modulation of cool and hot starspots, variable obscuration from an inner circumstellar disk, and changes in the mass accretion rate and other physical properties in a circumstellar disk as possible physical origins of the near-infrared variability. Cool spots alone can explain the observed variability characteristics in $\sim 56 \%-$ $77 \%$ of the stars, while the properties of the photometric fluctuations are more consistent with hot spots or extinction changes in at least $23 \%$ of the stars, and with variations in the disk mass accretion rate or inner disk radius in $\sim 1 \%$ of our sample. However, differences between the details of the observations and the details of variability predicted by hot-spot, extinction, and accretion disk models suggest either that another variability mechanism not considered here may be operative or that the observed variability represents the net results of several of these phenomena. Analysis of the star-count data indicates that the Orion Nebula cluster is part of a larger area of enhanced stellar surface density that extends over a $0.4 \times 2.4(3.4 \mathrm{pc} \times 20 \mathrm{pc})$ region containing $\sim 2700$ stars brighter than $K_{s}=14 \mathrm{mag}$.
\end{abstract}

Key words: infrared radiation - stars: individual (YY Orionis, BM Orionis) -

stars: pre-main-sequence - stars: variables: general

On-line material: additional figures, machine-readable tables

\section{INTRODUCTION}

Optical photometric variability is one of the original defining characteristics of pre-main-sequence stars (Joy 1945; Herbig 1962). Although pre-main-sequence objects are usually identified by other, less biased photometric and spectroscopic survey techniques now, modern variability observations remain a valuable probe of the stellar and circumstellar activity (e.g., Bouvier et al. 1993, 1995). Such monitoring studies have shown that photometric variability is a diverse phenomenon in that the observed flux can change by millimagnitudes to magnitudes on timescales of minutes to years, often with periodic, as well as aperiodic, components. For periodic stars, the variability is thought to originate mainly from cool magnetic or hot accretion spots on the stellar surface that are hundreds to thousands of kelvins different in temperature from the photosphere and rotate with the star. Aperiodic variability may arise from mechanisms such as coronal flares, irregular accretion of new material onto the star, and temporal variations in cir- cumstellar extinction (Herbst et al. 1994; see also review by Ménard \& Bertout 1999).

Young stellar objects are also variable at X-ray, ultraviolet, infrared, and radio wavelengths. Each of these wavelength regimes probes a different aspect of the young star and its circumstellar material, and they can be used together to establish a more comprehensive picture of the temporal properties and physical characteristics of young stellar objects (see, e.g., Kenyon et al. 1994; Guenther et al. 2000; Yudin 2000). Near-infrared monitoring observations, the focus of this study, are expected to probe relatively cool phenomena compared with optical, ultraviolet, and $\mathrm{X}$-ray studies and may probe phenomena not accessible to shorter wavelength studies, such as temperature, opacity, and geometry changes in the dust- and gas-rich circumstellar environment.

Variability at near-infrared wavelengths $(1.2-2.2 \mu \mathrm{m})$ has long been established from aperture photometry of young stars (Cohen \& Schwartz 1976; Rydgren \& Vrba 1983; 
Skrutskie et al. 1996) and extensive campaigns on individual targets (e.g., RU Lup and RY Tau, Hutchinson et al. 1989; SVS 13, Liseau, Lorenzetti, \& Molinari 1992 and Aspin \& Sandell 1994; DR Tau, Kenyon et al. 1994). More recent monitoring observations have used near-infrared arrays to study photometric variability of entire clusters of stars (e.g., Serpens; Horrobin, Casali, \& Eiroa 1997; Kaas 1999; Hodapp 1999). In addition to variability best modeled by cool spots, hot spots, and extinction variations, these studies have detected variable near-infrared emission that has been interpreted as originating from circumstellar material (Skrutskie et al. 1996). However, near-infrared variability is still not as well characterized as optical variability, and many questions remain as to the fraction of stars that exhibit such variability, the amplitudes and timescales of the photometric fluctuations, and the dominant physical mechanisms contributing to it. Establishing these properties is of interest in its own right but is also critical for understanding how to interpret single-epoch near-infrared photometry as representative of the mean flux levels in assessing the extinction, stellar mass and age, and accretion characteristics of individual stars.

In this contribution, we present an analysis of $J, H$, and $K_{s}$ time-series photometry over a $\sim 0.84 \times 6^{\circ}$ area centered on the Trapezium region of the Orion Nebula cluster (ONC) that encompasses the northern portion of the Orion A molecular cloud. Section 2 describes the full data set obtained for this study and the data reduction procedures. In $\S 3$, we identify the variable stars from the time-series photometry and present a sampling of the different types of observed variability. Sections 4 and 5 analyze the nearinfrared variability characteristics, and possible origins of the variability are discussed in $\S 6$. The implications of these results are discussed in $\S 7$, and our conclusions are summarized in $\S 8$.

\section{DATA}

\subsection{Observations and Data Processing}

The $J, H$, and $K_{s}$ observations of the Orion region were obtained with the Two Micron All Sky Survey (2MASS) $1.3 \mathrm{~m}$ telescope at Cerro Tololo, Chile, near the completion of the southern survey operations when auxiliary projects were scheduled in otherwise idle telescope time. All data were collected in standard 2MASS observing mode by scanning the telescope in declination to cover tiles of size $(\Delta \alpha$ $\times \Delta \delta) \sim\left(8.5 \times 6^{\circ}\right)$ in the three bands simultaneously. A 2MASS frame at each position within the tile consists of a doubly correlated difference of two NICMOS readouts separated by the $1.3 \mathrm{~s}$ frame integration time. The first readout occurs $51 \mathrm{~ms}$ after reset and independently provides a short integration to recover unsaturated images of bright ( 5-9 mag) stars. Each position on the sky is observed six times in this manner, for a total integration time of $7.8 \mathrm{~s}$. The nominal region surveyed for this project consists of seven contiguous tiles in right ascension as listed in Table 1, with each tile centered on a declination of $-6^{\circ}$. Adjacent tiles overlap by $\sim 40^{\prime \prime}-50^{\prime \prime}$ in right ascension, to provide a total sky coverage of $\sim 0.84 \times 6^{\circ}$. The seven tiles were observed on nearly a nightly basis for 16 nights in 2000 March. By the middle of March, Orion set early enough that it became necessary to observe fewer tiles per night, and by early April, just a single tile was observed. In total, data were obtained on 29 days in 2000 March-April over a
TABLE 1

OBSERVATIONAL COORDINATES

\begin{tabular}{|c|c|c|c|}
\hline \multirow[b]{2}{*}{ TiLe } & \multirow{2}{*}{$\begin{array}{c}\text { R.A. } \\
(\text { J2000.0) }\end{array}$} & \multicolumn{2}{|c|}{ NUMBER OF SCANS } \\
\hline & & Complete & Partial \\
\hline $1 \ldots \ldots$ & 053347 & 18 & 3 \\
\hline $2 \ldots \ldots$ & 053416 & 22 & 3 \\
\hline $3 \ldots \ldots$ & 053445 & 26 & 3 \\
\hline $4 \ldots \ldots$ & 053514 & 28 & 3 \\
\hline $5 \ldots \ldots$ & 053542 & 26 & 3 \\
\hline $6 \ldots \ldots$ & 053611 & 21 & 3 \\
\hline $7 \ldots \ldots$ & 053640 & 16 & 3 \\
\hline
\end{tabular}

Note.- Units of right ascension are hours, minutes, and seconds.

36 day time period, with the longest time gap in the observations being 4 days. The observing log for each tile is provided in Table 2 . In addition to these specially scheduled observations of the Orion region, the analysis conducted here incorporates data from normal 2MASS operations in 1998 March and 2000 February. With the above time sampling, the near-infrared photometric variability characteristics can be assessed on timescales of $\sim 1-36$ days, $\sim 2$ months, and $\sim 2$ years.

The data were reduced using a development version of the 2MASS data-processing pipeline at the Infrared Processing and Analysis Center (IPAC). This development pipeline is the same one that generated the data products for the first and second incremental 2MASS release catalogs. The data discussed in this paper, though, were not part of these incremental releases and will ultimately be replaced by the results of the final 2MASS processing. The 2MASS Explanatory Supplement (Cutri et al. 2000) ${ }^{1}$ contains complete details of the data reduction procedures, and only a brief summary is provided here. The output from the data reduction pipeline for each observed tile includes calibrated astronomical coordinates, $J, H$, and $K_{s}$ magnitudes, photometric uncertainties, and various photometric quality flags. Stars were identified in either the individual $51 \mathrm{~ms}$ images or a co-add of the six $1.3 \mathrm{~s}$ images using an algorithm operationally equivalent to DAOFIND (Stetson 1987). For bright stars ( $\$ 9 \mathrm{mag}$ ), photometry is obtained on the individual $51 \mathrm{~ms}$ images using an aperture radius of $4 "$ and a sky annulus that extends radially from $24^{\prime \prime}$ to $30^{\prime \prime}$. The final magnitude is computed by averaging the six aperture measurements and adopting the standard deviation of the mean as the photometric uncertainty. Stars brighter than $\sim 5 \mathrm{mag}$ are saturated in the short-exposure images. Photometry for stars fainter than $\sim 9$ mag is obtained by fitting a pointspread function (PSF) to the six $1.3 \mathrm{~s}$ images simultaneously. The PSF photometric uncertainty is computed from the Poisson noise within the PSF fitting radius and the observed fluctuations in the sky background. On rare occasions, the PSF fit did not converge in crowded regions and in areas with bright backgrounds such as the Orion Nebula. In such cases, an aperture magnitude is obtained on the $1.3 \mathrm{~s}$ integration images.

\footnotetext{
${ }^{1}$ See http://www.ipac.caltech.edu/2mass/releases/second/doc/ explsup.html.
} 
TABLE 2

LOG OF OBSERVATIONS

\begin{tabular}{|c|c|c|c|c|c|c|c|c|}
\hline UT Date & Tile 7 & Tile 6 & Tile 5 & Tile 4 & Tile 3 & Tile 2 & Tile 1 & Comments \\
\hline 1998 Mar $19 . . . .$. & $\mathrm{X}$ & $\mathrm{X}$ & $\mathrm{X}$ & $\mathrm{X}$ & $\mathrm{X}$ & $\mathrm{X}$ & $\mathrm{X}$ & $\delta>-6^{\circ}$ \\
\hline 1998 Mar $22 \ldots \ldots$ & $\mathrm{X}$ & $\mathrm{X}$ & $\mathrm{X}$ & $\mathrm{X}$ & $\mathrm{X}$ & $\mathrm{X}$ & $\mathrm{X}$ & $\delta<-6^{\circ}$ \\
\hline $2000 \mathrm{Feb} 6 \ldots \ldots$ & $\mathrm{X}$ & $\mathrm{X}$ & $\mathrm{X}$ & $\mathrm{X}$ & $\mathrm{X}$ & $\mathrm{X}$ & $\mathrm{X}$ & $\delta>-6^{\circ}$ \\
\hline $2000 \operatorname{Mar} 4 \ldots \ldots$ & $\mathrm{X}$ & $\mathrm{X}$ & $\mathrm{X}$ & $\mathrm{X}$ & $\mathrm{X}$ & $\mathrm{X}$ & $\mathrm{X}$ & \\
\hline $2000 \operatorname{Mar} 5 \ldots \ldots$ & $\mathrm{X}$ & $\mathrm{X}$ & $\mathrm{X}$ & $\mathrm{X}$ & $\mathrm{X}$ & $\mathrm{X}$ & $\mathrm{X}$ & \\
\hline $2000 \operatorname{Mar} 6 \ldots \ldots$ & $\mathrm{X}$ & $\mathrm{X}$ & $\mathrm{X}$ & $\mathrm{X}$ & $\mathrm{X}$ & $\mathrm{X}$ & $\mathrm{X}$ & \\
\hline $2000 \operatorname{Mar} 7 \ldots \ldots$ & $\mathrm{X}$ & $X$ & $\mathrm{X}$ & $\mathrm{X}$ & $\mathrm{X}$ & $\mathrm{X}$ & $\mathrm{X}$ & \\
\hline $2000 \operatorname{Mar} 9 \ldots \ldots$ & $\ldots$ & $\ldots$ & $\mathrm{X}$ & $\mathrm{X}$ & $\mathrm{X}$ & $\mathrm{X}$ & $\ldots$ & \\
\hline 2000 Mar $11 \ldots \ldots$ & $\mathrm{X}$ & $\mathrm{X}$ & $\mathrm{X}$ & $\mathrm{X}$ & $\mathrm{X}$ & $X$ & $\mathrm{X}$ & \\
\hline 2000 Mar $12 \ldots .$. & $\mathrm{X}$ & $X$ & $\mathrm{X}$ & $\mathrm{X}$ & $\mathrm{X}$ & $\mathrm{X}$ & $\mathrm{X}$ & \\
\hline 2000 Mar $13 \ldots . .$. & $\mathrm{X}$ & $\mathrm{X}$ & $\mathrm{X}$ & $\mathrm{X}$ & $\mathrm{X}$ & $\mathrm{X}$ & $\mathrm{X}$ & \\
\hline 2000 Mar $14 \ldots \ldots$ & $\mathrm{X}$ & $\mathrm{X}$ & $\mathrm{X}$ & $\mathrm{X}$ & $\mathrm{X}$ & $X$ & $\mathrm{X}$ & \\
\hline 2000 Mar $15 \ldots \ldots$ & $\mathrm{X}$ & $X$ & $\mathrm{X}$ & $\mathrm{X}$ & $\mathrm{X}$ & $\mathrm{X}$ & $\mathrm{X}$ & \\
\hline 2000 Mar $16 \ldots .$. & $\mathrm{X}$ & $\mathrm{X}$ & $\mathrm{X}$ & $\mathrm{X}$ & $\mathrm{X}$ & $\mathrm{X}$ & $\mathrm{X}$ & \\
\hline 2000 Mar $18 \ldots \ldots$ & $\mathrm{X}$ & $\mathrm{X}$ & $\mathrm{X}$ & $\mathrm{X}$ & $\mathrm{X}$ & $\mathrm{X}$ & $\mathrm{X}$ & \\
\hline 2000 Mar $19 \ldots . .$. & $\mathrm{X}$ & $X$ & $\mathrm{X}$ & $\mathrm{X}$ & $\mathrm{X}$ & $\mathrm{X}$ & $\mathrm{X}$ & \\
\hline 2000 Mar $20 \ldots . .$. & $\mathrm{X}$ & $\mathrm{X}$ & $\mathrm{X}$ & $\mathrm{X}$ & $\mathrm{X}$ & $\mathrm{X}$ & $\mathrm{X}$ & \\
\hline 2000 Mar $21 \ldots .$. & $\mathrm{X}$ & $\mathrm{X}$ & $\mathrm{X}$ & $\mathrm{X}$ & $\mathrm{X}$ & $\mathrm{X}$ & $\mathrm{X}$ & \\
\hline 2000 Mar $22 \ldots . .$. & $\mathrm{X}$ & $\mathrm{X}$ & $\mathrm{X}$ & $\mathrm{X}$ & $\mathrm{X}$ & $\mathrm{X}$ & $\mathrm{X}$ & \\
\hline 2000 Mar $23 \ldots \ldots$ & $\mathrm{X}$ & $\mathrm{X}$ & $\mathrm{X}$ & $\mathrm{X}$ & $\mathrm{X}$ & $\mathrm{X}$ & $\mathrm{X}$ & \\
\hline 2000 Mar $24 \ldots \ldots$ & $\ldots$ & $\mathrm{X}$ & $\mathrm{X}$ & $\mathrm{X}$ & $\mathrm{X}$ & $X$ & $\mathrm{X}$ & \\
\hline 2000 Mar $25 \ldots . .$. & $\ldots$ & $\mathrm{X}$ & $\mathrm{X}$ & $\mathrm{X}$ & $\mathrm{X}$ & $\mathrm{X}$ & $\mathrm{X}$ & \\
\hline 2000 Mar $26 \ldots . .$. & $\ldots$ & $\mathrm{X}$ & $\mathrm{X}$ & $\mathrm{X}$ & $\mathrm{X}$ & $\mathrm{X}$ & $\ldots$ & \\
\hline 2000 Mar $27 \ldots \ldots$ & $\ldots$ & $\mathrm{X}$ & $\mathrm{X}$ & $\mathrm{X}$ & $\mathrm{X}$ & $X$ & $\ldots$ & \\
\hline 2000 Mar $28 \ldots . .$. & $\ldots$ & $\mathrm{X}$ & $\mathrm{X}$ & $\mathrm{X}$ & $\mathrm{X}$ & $\mathrm{X}$ & $\ldots$ & \\
\hline 2000 Mar $30 \ldots .$. & $\ldots$ & $\ldots$ & $\mathrm{X}$ & $\mathrm{X}$ & $\mathrm{X}$ & $\ldots$ & $\ldots$ & \\
\hline 2000 Mar $31 \ldots \ldots$ & $\ldots$ & $\ldots$ & $\mathrm{X}$ & $\mathrm{X}$ & $\mathrm{X}$ & $\ldots$ & $\ldots$ & \\
\hline 2000 Apr $1 \ldots \ldots \ldots$ & $\ldots$ & $\ldots$ & $\mathrm{X}$ & $\mathrm{X}$ & $\mathrm{X}$ & $\ldots$ & $\ldots$ & \\
\hline 2000 Apr $5 \ldots \ldots \ldots$ & $\ldots$ & $\ldots$ & $\ldots$ & $\mathrm{X}$ & $\mathrm{X}$ & $\ldots$ & $\ldots$ & \\
\hline 2000 Apr $6 \ldots \ldots \ldots$ & $\ldots$ & $\cdots$ & $\ldots$ & $\mathrm{X}$ & $\ldots$ & $\ldots$ & $\cdots$ & \\
\hline 2000 Apr $7 \ldots \ldots \ldots$ & $\ldots$ & $\ldots$ & $\ldots$ & $\mathrm{X}$ & $\ldots$ & $\ldots$ & $\ldots$ & \\
\hline 2000 Apr $8 \ldots \ldots \ldots$ & $\ldots$ & $\ldots$ & $\mathrm{X}$ & $\ldots$ & $\ldots$ & $\ldots$ & $\ldots$ & \\
\hline
\end{tabular}

The instrumental magnitudes were calibrated by observation of a 2MASS standard field every hour as part of normal 2MASS operations, with typical $1 \sigma$ calibration uncertainties in the nightly photometric zero points of $\lesssim 0.015 \mathrm{mag}$ in each band. For our time-series data, the internal accuracy of the photometry can be improved by defining a grid of bright, isolated stars within the survey region as secondary standards and assuming that their average magnitudes as an ensemble do not change in time. These secondary standards were selected as isolated stars more than $20^{\prime \prime}$ from the nearest object that are brighter than 14 mag in each band with photometric rms scatter from the repeated observations less than 0.1 mag. A total of 3649 stars satisfied these criteria. The fiducial magnitude for each secondary standard was defined as the average magnitude computed using all available observations. The average photometric offset for each tile relative to this secondary grid was then computed, with a statistical accuracy of $\sim 0.002 \mathrm{mag}$. The applied photometric offsets were typically less than 0.015 mag per band per tile, except for the 1998 data, for which the offsets were as large as 0.038 mag. The 1998 March observations were some of the first observations conducted with the southern 2MASS telescope and were obtained and calibrated before the complete 2MASS standard calibration grid for the southern survey had been established.

Time-series photometry for each star was produced by matching point sources with positions coincident to within $1^{\prime \prime}$. Sources were merged in this manner from night to night and also within a night to account for those stars in the overlap regions between adjacent tiles. The Julian Date for each observation was estimated from the starting Julian Date of the tile and assuming that the scan rate $\left(\sim 1^{\circ}\right.$ minute ${ }^{-1}$ ) along the $6^{\circ}$-long tile was constant.

\subsection{Point-Source List}

As mentioned in the previous section, not all tiles in our image data were observed on all nights. To establish as uniform, reliable, and complete a source list as possible over the entire region surveyed, the 16 nights in 2000 March common to all seven right ascension tiles were used to define the areal completeness limits, the magnitude completeness limits, and the final source list.

First, the maximum angular area over which the observations are spatially complete was determined by examining the spatial distribution of sources detected on all 16 nights. In J2000.0 equatorial coordinates, this area encompasses the right ascension range 83.405 to 84.250 and the declination range -8.98 to -2.88 , for a total area of 5.12 $\mathrm{deg}^{2}$. Second, the magnitude completeness limits at $J, H$, and $K_{s}$ were assessed using all stars with at least eight detections in the appropriate band and no artifact or confusion flags from the IPAC pipeline processing. The requirement that the star be detected at least eight times in the time series is arbitrarily set to remove transient detections (e.g., meteor trails) and to reduce the number of double stars that 
are resolved on only a fraction of the nights because of differences in seeing conditions. Stars between $-6^{\circ}$ and $-5^{\circ}$ declination were excluded from the completeness analysis to avoid complications in identifying point sources in the Orion Nebula and associated dense cluster. For our data, the magnitude above which a star is detected on at least 15 of the 16 nights in the absence of source confusion occurs at $J=16.0, H=15.4$, and $K_{s}=14.8$. The $5.12 \mathrm{deg}^{2}$ area of spatial completeness contains 18,552 stars with at least eight detections brighter than or equal to these defined magnitude completeness limits in one or more bands.

The preliminary source list thus consisted of 18,552 stars, $\sim 93 \%$ of which have no artifact or confusion flags from the processing pipeline in any of the observations. After removing those sources flagged as persistence or filter glints, potential lingering artifacts were identified as objects that either had unusual stellar colors, were detected fewer than 15 times, or had flags indicating contaminated or confused photometry from a nearby star. Many, but not all, of these $\sim 1300$ sources were visually inspected in the images. Most of the objects have processing flags indicating that the source either is an artifact or is real but near a diffraction spike or potentially confused with a nearby bright star. In 2MASS public data releases, many of these sources have been omitted to ensure reliability of the output catalogs. For this project, however, we used the repeated nature of our observations and visual inspection of the images to formulate criteria to distinguish real sources from the artifacts among the objects with processing flags. This analysis enhanced the spatial completeness of the observations while retaining a reliable source list. From inspection of the images for many sources, it became apparent that real sources are generally detected on every night and usually in all three bands. Sources detected fewer than 15 times but without any flags set are generally double sources resolved on only a fraction of the nights, or sources near the completeness limits but not detected on every apparition. Artifacts, if detected on all 16 nights, are usually present in only one band even though the upper limits to the stellar flux reported in the IPAC processing indicate that the source should have been detected in the other two bands. Artifacts detected in all three bands can also be distinguished by their unusual colors. These criteria were then used to identify and remove artifacts from the source list.

Finally, point-source detection in regions with bright, extended backgrounds such as the Orion Nebula are notoriously difficult. Therefore, in the core of the Orion Nebula the 2MASS source list was compared with the deep $(K \sim 17.5 \mathrm{mag})$, high-resolution Keck image of the inner $5^{\prime} \times 5^{\prime}$ of the ONC presented by Hillenbrand \& Carpenter (2000). While the 2MASS and Keck images were obtained $\sim 1$ year apart and stars could in principle be present in the 2MASS images but not in the Keck data as a result of variability, in practice none of the 2MASS-only sources looked to be a convincing point source from visual inspection of the images. We therefore assumed that any source present in the 2MASS data but not detected in the Keck images is likely a knot in the nebulosity and removed it from the source list.

In summary, of the 18,552 stars meeting the spatial and magnitude completeness criteria, 744 were deemed artifacts around bright stars or knots of nebulosity in the Orion Nebula and were removed from the source list. The final source list for our variability analysis contains 17,808 stars brighter than the defined completeness limits $(J=16.0$, $\left.H=15.4, K_{s}=14.8\right)$ in at least one band and within the angular area $\alpha=83.405$ to 84.250 and $\delta=-8.98$ to $-2.88(\mathrm{~J} 2000.0)$.

\subsection{Photometric Integrity}

With the point-source list established, our next step was to ensure photometric integrity by removing all photometry suspected of being unreliable so that true photometric fluctuations could be distinguished from spurious individual measurements. Unreliable photometry can result either from characteristics that prevent good photometry from ever being obtained for a particular real point source (e.g., a neighboring bright star, a stellar companion, bright nebulosity) or from conditions that may affect a single measurement (e.g., a cosmic-ray hit, meteor trail, a PSF fit that did not converge). Based on visual inspection of many sources, the following criteria were adopted to formulate a list of reliable photometric measurements: First, only photometric measurements obtained using aperture photometry in the $51 \mathrm{~ms}$ frames or PSF-fitting photometry in the $1.3 \mathrm{~s}$ images (see $\S 2.1$ ) were used. Second, $1.8 \%$ of all objects in the source list were repeatedly identified as extended at the resolution of 2MASS, as the $\chi^{2}$ from the PSF fit averaged over all apparitions of the object exceeded 1.5 in each band. As described in $\S 3.1$, these sources were not considered for inclusion in the uniformly selected list of variable stars, but a few of these extended sources were identified as variable objects using subjective criteria. Finally, to remove potentially unreliable individual photometric measurements from the time-series data for a given star, any individual photometric measurement based on PSF fitting that had a resulting $\chi^{2}>3.0$ was not used in judging the photometric variability.

We tested that the estimated photometric uncertainties (see $\S 2.1$ ) accurately reflect the real noise characteristics of the 2MASS photometry using the reduced $\chi^{2}\left(\chi_{v}^{2}\right)$, computed for each band and each star as

$$
\chi_{v}^{2}=\frac{1}{v} \sum_{i=1}^{n} \frac{\left(m_{i}-\bar{m}\right)^{2}}{\sigma_{i}^{2}},
$$

where $n$ is the number of reliable photometric measurements as defined above, $v=n-1$ is the number of degrees of freedom, and $\sigma_{i}$ is the estimated photometric uncertainty. Histograms of $\chi_{v}^{2}$ are shown in Figure 1 for the $J, H$, and $K_{s}$ photometry on the 16 nights common to the entire survey area. The solid curves show the expected $\chi_{v}^{2}$ distribution for 15 degrees of freedom normalized to the total number of stars. (In practice, $\sim 20 \%$ of the stars may have more or fewer than 16 measurements, depending on whether the star is in the overlap region of adjacent tiles or if some of the individual photometric measurements were discarded as just described.) Figure 1 shows that the observed and expected $\chi_{v}^{2}$ distributions agree rather well for the majority of the stars, demonstrating that the estimated photometric uncertainties accurately reflect the expected photometric scatter due to random noise. A number of stars have significantly larger values of $\chi_{v}^{2}$ than expected for random noise. As discussed in $\S 3.1$, the majority of these objects are true variable stars.

To estimate the signal-to-noise ratio of the photometry, Figure 2 plots the observed photometric rms $\left(\sigma_{\text {obs }}\right)$ in the time series for each star as a function of magnitude for 


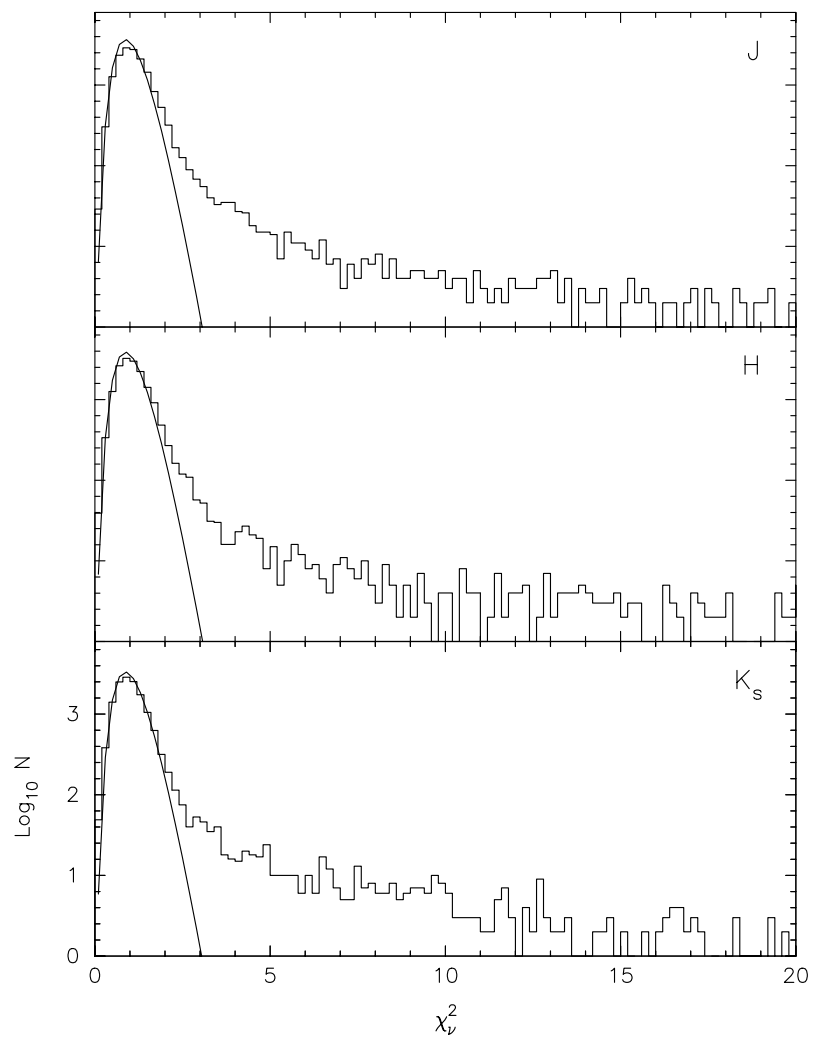

FIG. 1.- Histogram of the reduced $\chi^{2}\left(\chi_{v}^{2}\right)$ for the observed photometric deviations about the mean for the $J-, H-$, and $K_{\mathrm{s}}$-band photometry from the 16 nights in which all seven tiles were observed. The solid curve in each panel is the expected $\chi_{v}^{2}$ distribution for 15 degrees of freedom, where the curves have been normalized by the total number of stars. This figure demonstrates that the observed photometric scatter for the majority of the stars is consistent with random noise. Stars with large values of $\chi_{v}^{2}$ are candidate variable stars.

objects brighter than the completeness limits. The observed rms in the time-series photometry was computed from the individual magnitudes $\left(m_{i}\right)$ and photometric uncertainties $\left(\sigma_{i}\right)$ using

$$
\sigma_{\mathrm{obs}}^{2}=\frac{n \sum_{i=1}^{n} w_{i}\left(m_{i}-\bar{m}\right)^{2}}{(n-1) \sum_{i=1}^{n} w_{i}},
$$

where $w_{i}=1.0 / \sigma_{i}^{2}$ is the weight assigned to each observation. We also define the expected photometric rms $\left(\sigma_{\text {noise }}\right)$ in the time-series data due to random noise using

$$
\sigma_{\text {noise }}^{2}=\frac{n}{\sum_{i=1}^{n}\left(1.0 / \sigma_{i}^{2}\right)} .
$$

Figure 2 shows a correlation with magnitude, as expected if the observed rms in the time series is mostly due to photometric noise and not to intrinsic variability. The observed rms values range from a minimum of $\sim 0.015 \mathrm{mag}$ for the bright stars to $\lesssim 0.15$ mag for stars near the completeness limit. The observed rms floor of $\sim 0.015 \mathrm{mag}$ for the brighter stars is interpreted as the minimum photometric repeatability for these data, and consequently, a minimum photometric uncertainty of $0.015 \mathrm{mag}$ has been imposed on all of the photometric measurements. Based on the estimated photometric uncertainties produced by the IPAC data reduction pipeline, we find that $97 \%, 89 \%$, and $86 \%$ of the stars at $J, H$, and $K_{s}$, respectively, have a signal-to-noise

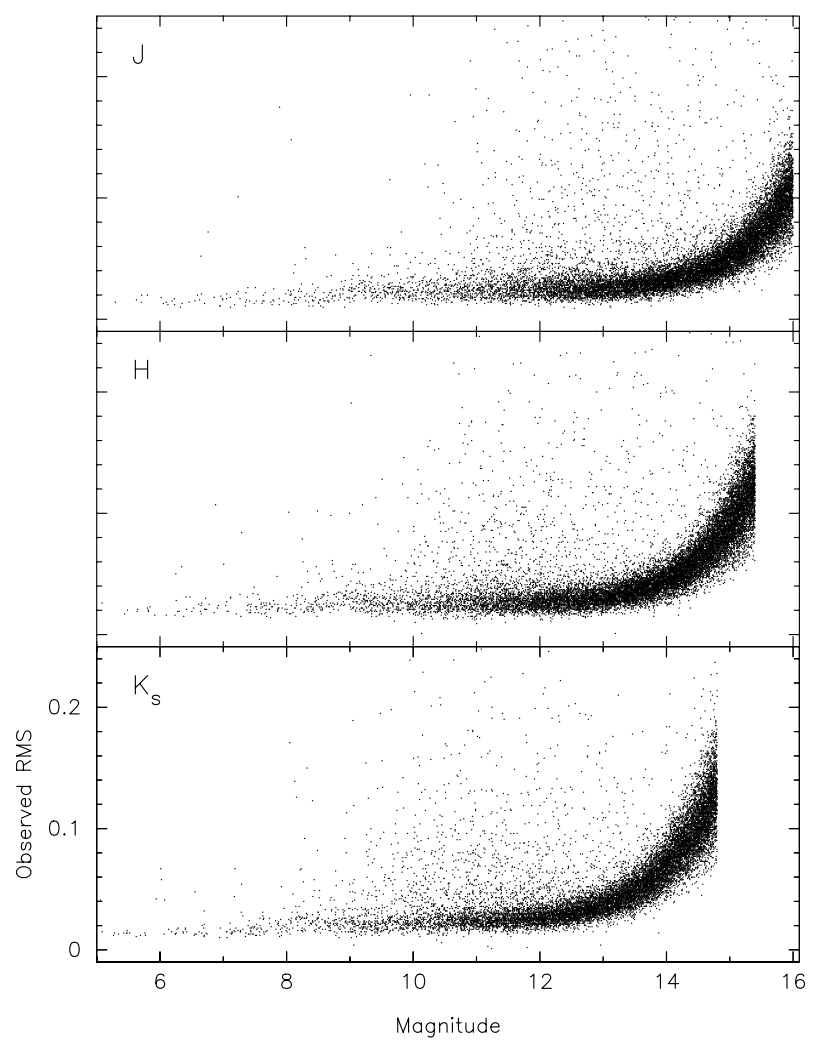

FIG. 2.-Observed photometric rms in the time-series data as a function of magnitude for stars brighter than the defined completeness limits. The observed rms ranges from $\sim 0.015 \mathrm{mag}$ for the brightest stars to $\lesssim 0.15$ mag (i.e., signal-to-noise ratio $\geq 7$ ) for stars at the completeness limit.

ratio per measurement $\geq 10$, and $99 \%$ have a signal-tonoise ratio $\geq 7$.

\section{VARIABLE STARS IN THE ORION A MOLECULAR CLOUD}

\subsection{Identification}

Operationally, a variable star is a star that exhibits larger photometric variations over the course of a time series than expected based on the photometric uncertainties. Several techniques have been utilized in the literature to identify variable stars, each with its own merits and limitations. Historically, variable stars were identified through visual inspection of image data or light curves. This approach has the advantage that different types of variability can be identified visually that may be difficult to pick out in an automated fashion, and suspicious photometric measurements can be eliminated by inspection of images. However, the subjective nature of this process and the large number of stars in our survey make this an impractical option (although we did look at several thousand light curves!), and more quantitative measures of the variability were examined.

One quantitative estimator of photometric variability is the reduced $\chi^{2}\left(\chi_{v}^{2}\right)$ of the observed magnitudes (see eq. [1]), which directly translates into a probability that the observed variations could have resulted from Gaussian noise (Bevington 1969). The $\chi_{v}^{2}$ technique is amenable to multiband photometry in that the probability that a high $\chi_{v}^{2}$ is observed in two or more bands can be readily computed. The disadvantage to this approach, though, is that noise is often non-Gaussian in confused regions, and a single "bad" 
measurement can cause a high $\chi_{v}^{2}$ despite efforts to remove such observations. Further, the $\chi_{v}^{2}$ statistic does not take advantage of correlated changes in multiband magnitudes as a function of time, which can be used to identify variables with a low amplitude. These considerations motivated Welch \& Stetson (1993; see also Stetson 1996) to propose an alternate statistic that correlates the photometric fluctuations in multiband photometry. Although the advantages are exactly the limitations of the $\chi_{v}^{2}$ technique, the main difficulties with this alternate variability index are that its mathematical properties are not as well understood as $\chi_{v}^{2}$ (except through Monte Carlo simulations) and it dilutes the signatures of variability occurring only in a single band.

After examining the results from each of the three techniques described above, it became apparent that a single criterion could not be implemented to identify all variable stars, and a combination of these methods was therefore used. First, a uniformly selected list of variable stars was created using the Stetson variability index, $J$, as defined in Stetson (1996), but renaming the index $S$ to avoid confusion with the 2MASS $J$-band magnitude. Much of the analysis of variable star properties that follows uses this uniformly selected list. The Stetson variability index was computed for each star from the observed $J, H$, and $K_{s}$ magnitudes and associated photometric uncertainties as

$$
S=\frac{\sum_{i=1}^{p} g_{i} \operatorname{sgn}\left(P_{i}\right) \sqrt{\left|P_{i}\right|}}{\sum_{i=1}^{n} g_{i}}
$$

where $p$ is the number of pairs of observations for a star taken at the same time, $P_{i}=\delta_{j(i)} \delta_{k(i)}$ is the product of the normalized residuals of two observations, and $g_{i}$ is the weight assigned to each normalized residual (see Stetson 1996). The normalized residual for a given band, $\delta_{i}$, is computed as

$$
\delta_{i}=\sqrt{\frac{n}{n-1}} \frac{m_{i}-\bar{m}}{\sigma_{i}},
$$

where $n$ is the number of measurements used to determine the mean magnitude $\bar{m}$ and $\sigma_{i}$ is the photometric uncertainty (see $\S 2.3$ ). In the case of a star with a measurement in only one time sample, the product of the normalized residuals was set to $P_{i}=\delta_{j(i)}^{2}-1$. The formula for $P_{i}$ is different for stars with only a single measurement since the expectation value for $\delta_{j(i)}^{2}$ is 1 for random, Gaussian noise. The weights, $g_{i}$, are set based on the number of detections in a given time sample. Time samples with three band detections were assigned a weight of $\frac{2}{3}$ (implying a total weight of 2.0 for the three possible pair combinations), while one- or two-band detections were assigned a weight of 1 .

Figure 3 shows the Stetson statistic as a function of the $H$-band magnitude for photometry obtained on the 16 nights common to the entire survey area. For random noise, the Stetson variability index should be scattered around zero and have higher, positive values for stars with correlated variability. For this data set, the Stetson variability index has a positive value on average for the brighter stars. The origin of this offset is unclear, but it suggests that a weak correlation exists between the $J, H$, and $K_{s}$ photometry, possibly from the fact that the three bands were observed simultaneously. Nonetheless, the Stetson variability index is skewed toward large positive values around the nominal value, indicating that a number of stars exhibit real

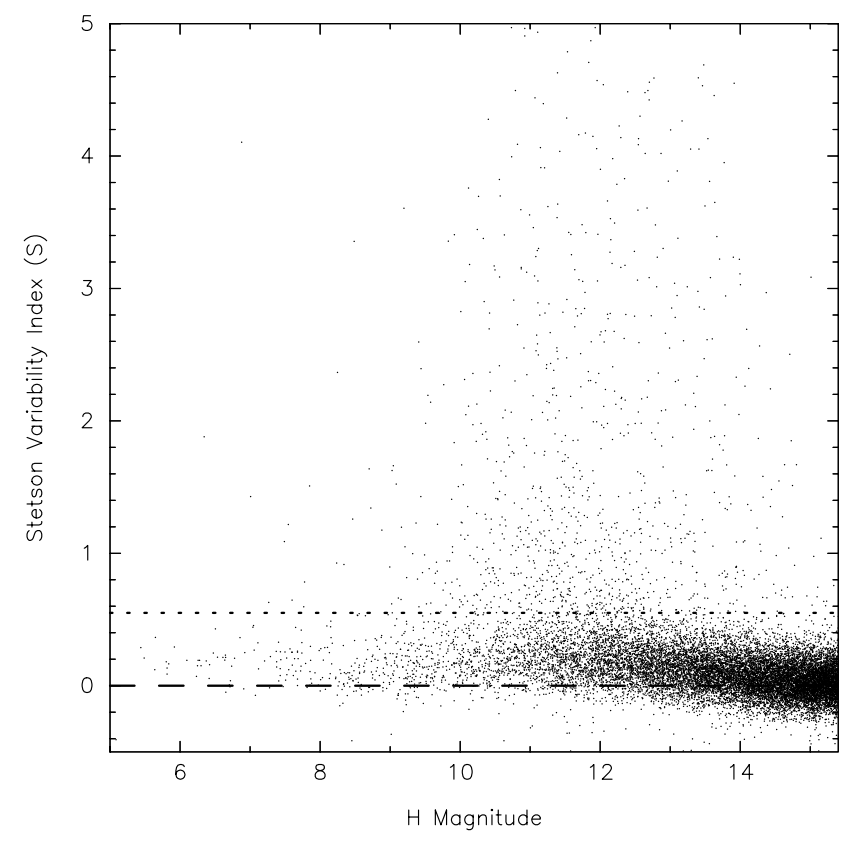

FIG. 3.-Stetson variability index $(S)$ plotted as a function of $H$ magnitude for stars brighter than $H=15.4$. Only data for stars in the 16 nights common to all seven tiles were used to compute the variability index. The dashed line at $S=0$ shows the expected value of the variability index for nonvariable stars, and the dotted line at $S=0.55$ represents the minimum adopted value used to identify variable stars in this study. The origin of the positive bias in the computed index values is unknown and suggests that a weak correlation exists between the $J, H$, and $K_{s}$ photometry, possibly from the fact that the three bands were observed at the same time. Note that 53 stars with $S>5.0$ are not shown, for clarity.

variability that is correlated between the three 2MASS bands. The minimum value of the Stetson variability index, which likely represents real variability as opposed to random noise, was estimated by visually examining the light curves as a function of the Stetson index and plotting the Stetson index versus the observed $\chi_{v}^{2}$. Based on this analysis, variable stars were defined as objects having a Stetson index $S \geq 0.55$.

The Stetson index was computed as described above for all stars in the sample for two time periods. In sample 1, the 16 nights in common to the entire survey area were used to select a spatially complete set of variable stars based on identical temporal sampling. In sample 2, all of the 2000 March-April data were used, to obtain a broader assessment of the variability characteristics on timescales up to $\sim 1$ month. (The $\sim 2$ month and 2 year variability characteristics derived from inclusion of the 2000 February and 1998 March data are considered below.) All light curves and images for each candidate variable star were visually examined, and 47 stars were removed because the photometry was deemed suspect as a result of a nearby bright star or bright nebulosity from the Orion Nebula. As summarized in Table 3, a total of 1006 variable stars were identified in sample 1, and 1054 stars in sample 2 . These two samples largely overlap, as just 72 variables are identified only in sample 1, and 120 only in sample 2 . The light curves for stars identified as variable in only one of the time samples were examined, and indeed many appear variable for only a portion of the light curve or have Stetson indexes close to the adopted threshold, but exceeding or falling short of the threshold depending on which data were included. While 
TABLE 3

STELLAR SAMPLES

\begin{tabular}{|c|c|}
\hline Sample & $N_{\text {stars }}$ \\
\hline All stars ${ }^{\mathrm{a}} .$. & 17808 \\
\hline All variables ... & 1235 \\
\hline Sample 1 variables $^{\mathrm{b}}$ & 1006 \\
\hline Sample 2 variables ${ }^{\mathrm{c}}$ & 1054 \\
\hline 2 month variables ${ }^{\mathrm{d}}$ & 14 \\
\hline 2 year variables ${ }^{\mathrm{e}}$. & 72 \\
\hline Periodic ............ & 233 \\
\hline Eclipsing candidates $\ldots \ldots \ldots \ldots \ldots \ldots \ldots \ldots$ & 22 \\
\hline Subjective additions .................... & 60 \\
\hline
\end{tabular}

${ }^{\text {a }} J \leq 16.0 \mathrm{mag}, H \leq 15.4 \mathrm{mag}$, or $K_{s} \leq 14.8 \mathrm{mag}$.

${ }^{b}$ Identified from 16 nights common to all tiles.

${ }^{c}$ Identified from 2000 March-April observations.

d Between 2000 February and 2000 March-April.

e Between 1998 March and 2000 March-April.

the observed photometric $\chi_{v}^{2}$ did not formally enter into the selection criteria, $\sim 90 \%$ of the stars in both samples have $\chi_{v}^{2}$ values indicating that there is less than a $0.1 \%$ chance that the observed fluctuations could be due to random, Gaussian noise. The observed $\chi_{v}^{2}$ distributions suggest that fewer than $1 \%$ of the identified variables are likely to be spurious as a result of random noise, although the contamination rate in practice is likely somewhat higher because of non-Gaussian noise (e.g., nebulosity, source confusion) in some regions.

To evaluate whether our selection criteria for photometric variability were too stringent or too liberal, we estimated the number of variable stars we should detect based on the statistics of the observed and expected $\chi_{v}^{2}$ distributions shown in Figure 1. Assuming for the moment that only stars with $\chi_{v}^{2} \geq 2$ are variables, and subtracting the expected $\chi_{v}^{2}$ distribution from the observed distribution, the predicted number of variable stars is $\sim 900$ at $K_{s}$ band and $\sim 1300$ at $J$ band. Compared with $\sim 1100$ variables actually identified in our analysis, we conclude that our adopted criteria with regard to the Stetson index have identified approximately the number of variables expected given the noise characteristics of the data and the observed photometric fluctuations.

In addition to the variable star sample selected strictly according to the Stetson index, we also include stars in our variable star list that have sufficiently impressive variability as judged from visual inspection of light curves but which did not meet the imposed Stetson index and PSF thresholds. Light curves were identified for visual inspection based on one or more of the following quantitative criteria: (1) large $\chi_{v}^{2}$ in one or more bands but otherwise a small Stetson index, (2) long-term photometric variations, as defined in $\S 5.3 .1$, (3) significant periods in a Lomb-Scargle analysis despite small Stetson index (see $\S$ 5.3.2), and (4) sources extended at the 2MASS resolution, but having a large Stetson index. These somewhat arbitrary additions constitute a small percentage $(\sim 10 \%)$ of the final variable star list.

Table 4 summarizes the photometric properties of the 1235 variable stars identified from our data. Included in the table are an identification number, the equatorial J2000.0 coordinates, the average $J, H$, and $K_{s}$ magnitudes, the observed photometric rms in each, the number of highquality photometric measurements used to assess the variability, the Stetson variability index, and a six-digit flag that indicates the variability exhibited by the star. The photometric information and Stetson index reported in this table were computed using all available photometry meeting the criteria in $\S 2.3$. Each digit in the variability flag represents a different variability indicator and is set to " 1 " if the star exhibits that type of variability, and set to " 0 " otherwise. In order starting from the leftmost, the digits represent (1) variability in the 16 nights common to the survey area as indicated by the Stetson index, (2) variability identified in the entire 2000 March-April time series as indicated by the Stetson index, (3) long-term variability relative to either the 1998 March or 2000 February data (see $\S 5.3 .1$ ), (4) periodicity as determined from the Lomb-Scargle periodogram analysis (see $\S 5.3 .2$ ), (5) a candidate eclipsing system (see $\S 6.4$ ), and (6) variability identified from subjective, visual inspection of the light curves, usually among sources that appear extended at the 2MASS resolution. Note that one or more of the first five digits in the variability flag may be set for any individual star.

TABLE 4

NEAR-INFRARED VARIABLE STARS

\begin{tabular}{|c|c|c|c|c|c|c|c|c|c|c|c|c|c|}
\hline \multirow[b]{2}{*}{ ID } & \multicolumn{2}{|c|}{ J2000.0 EQUATORIAL (deg) } & \multicolumn{3}{|c|}{ MAGNITUDES $^{\mathbf{a}}$} & \multicolumn{3}{|c|}{ OBSERVED RMS ${ }^{\mathrm{a}}$} & \multicolumn{3}{|c|}{$N$} & \multicolumn{2}{|c|}{ VARIABILITY } \\
\hline & $\alpha$ & $\delta$ & $J$ & $H$ & $K_{s}$ & $J$ & $H$ & $K_{s}$ & $J$ & $H$ & $K_{s}$ & Index & Flag $^{\mathrm{b}}$ \\
\hline 91. & 83.410336 & -6.000067 & 13.724 & 13.086 & 12.814 & 0.035 & 0.031 & 0.043 & 22 & 21 & 21 & 0.46 & 110000 \\
\hline $123 \ldots \ldots$ & 83.412085 & -7.502039 & 11.428 & 10.809 & 10.573 & 0.055 & 0.063 & 0.056 & 19 & 19 & 19 & 0.89 & 000001 \\
\hline 237. & 83.418483 & -3.056776 & 14.113 & 13.511 & 13.126 & 0.053 & 0.058 & 0.086 & 21 & 21 & 21 & 0.79 & 110000 \\
\hline $292 .$. & 83.421295 & -3.224478 & 14.872 & 14.484 & 14.351 & 0.113 & 0.120 & 0.146 & 20 & 20 & 20 & 1.09 & 110000 \\
\hline $307 \ldots \ldots$ & 83.421983 & -4.823802 & 12.192 & 11.525 & 11.292 & 0.035 & 0.039 & 0.037 & 20 & 20 & 20 & 0.87 & 110000 \\
\hline
\end{tabular}

NoTE.-Table 4 is presented in its entirety in the electronic edition of the Astronomical Journal. A portion is shown here for guidance regarding its form and content.

${ }^{\text {a }}$ Photometric parameters computed using all available data.

b Each digit represents a different variability characteristic; from first digit to last,

1. Variable on 16 nights common to entire area;

2. Variable in 2000 March-April data;

3. Long-term variability in 2000 February, 1998 March, or both;

4. Periodic variable;

5. Eclipsing candidate; and

6. Selected as variable from subjective inspection of light curves. 

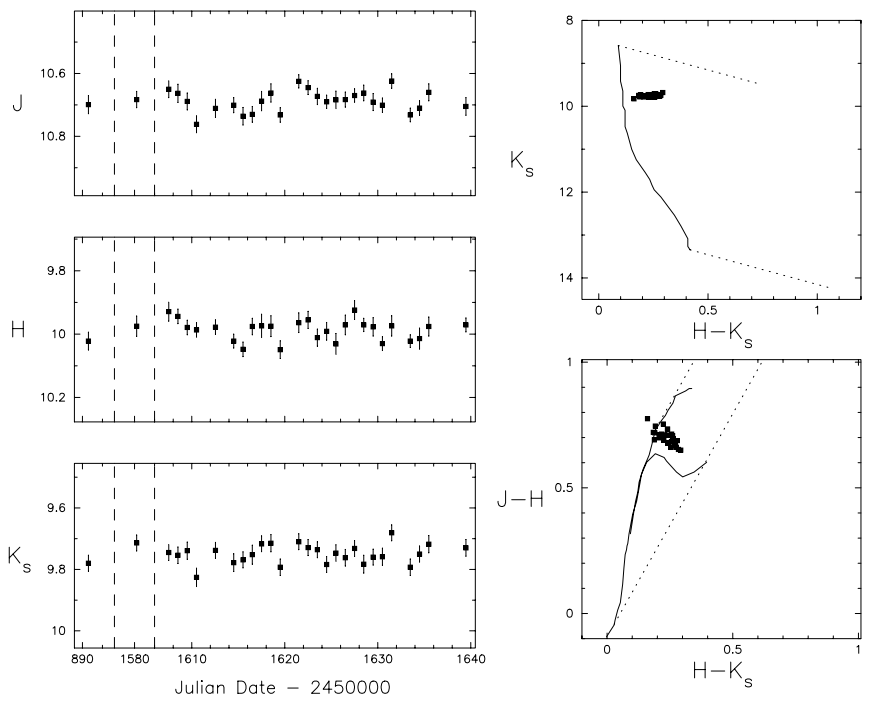

FIG. 4.-Photometric data for star 5123, which has a Stetson variability index $(S=0.58)$ that is just above the limit $(0.55)$ to be classified as a variable. The left panels show the $J, H$, and $K_{s}$ light curves. The first data point in each light curve is from 1998 March, the second data point from 2000 February, and the remaining photometry from 2000 March-April. The vertical bars through the data points represent the $\pm 1 \sigma$ photometric uncertainties. The right panels show the $K_{s}$ vs. $H-K_{s}$ color-magnitude diagram and the $J-H$ vs. $H-K_{s}$ color-color diagram for each data point in the time series, where the dotted lines represent the interstellar reddening vector from Cohen et al. (1981) transformed into the 2MASS photometric system (Carpenter 2001). The uncertainties in the photometric measurements have been omitted for clarity. The solid line in the colormagnitude diagram is the $1 \mathrm{Myr}$ pre-main-sequence isochrone from D’Antona \& Mazzitelli (1997) for stellar masses between 0.08 and $3 M_{\odot}$. The solid curves in the color-color diagram are the loci of red giant and main-sequence stars from Bessell \& Brett (1988) in the 2MASS color system.

\subsection{Light Curves}

For each variable star listed in Table 4, a figure has been generated showing the $J, H$, and $K_{s}$ light curves and the $K_{s}$ versus $H-K_{s}$ color-magnitude diagram and $J-H$ versus $H-K_{s}$ color-color diagram for the time-series data. It is not feasible to present figures for all 1235 variable stars here, but figures for all variable stars are available in the electronic version of this article. (The electronic versions of the figures also include the $J-H$ and $H-K$ light curves, the $J$ vs. $J-H$ color-magnitude diagram, postage-stamp $J, H$, and $K_{s}$ images, and a tabular summary of the photometric data.) Further, GIF images for each star, along with search capabilities by object name or coordinates, links to tabular data, and cross-references to existing optical and nearinfrared catalogs are also currently available at J. M. C.'s World Wide Web site. ${ }^{2}$ Many of the stars display unique variability characteristics that can only be appreciated from inspection of these figures.

Figures 4-13 present a sampling of the observed nearinfrared variability characteristics. Figure 4 shows a star with a Stetson index of 0.58 (just above the adopted threshold of 0.55 to identify variable stars) that exhibits correlated, low-amplitude magnitude changes in all three bands. Figure 5 illustrates a star with periodic, nearly sinusoidal, colorless

\footnotetext{
${ }^{2}$ See http://www.astro.caltech.edu/ jmc/variables/orion.
}

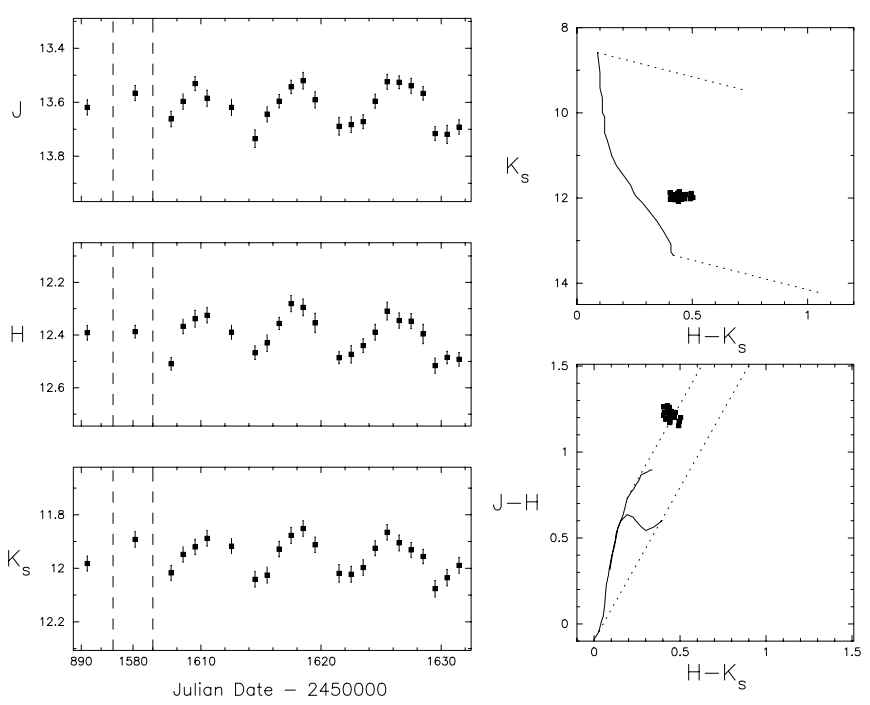

FIG. 5.-Photometric data for star 3730 , classified as a periodic star based on the Lomb-Scargle periodogram analysis (see § 5.3.2). The derived period in each band is $\sim 8$ days.

variations with a peak-to-peak amplitude of $\sim 0.25 \mathrm{mag}$ in each band. Figure 6 also shows a periodic system in which the photometric fluctuations do not occur smoothly in time, but on discrete days, as expected for an eclipsing binary system. As opposed to the relatively rapid fluctuations illustrated thus far, Figure 7 shows a star that continuously brightened over the 2000 March-April time period. The brightness of the star, however, did not change significantly between 1998 March and 2000 March, suggesting that these variations are not a long-term trend.

Some of the larger amplitude variables also display significant color variations. Figure 8 shows a star (YY Ori) in which the photometric fluctuations are largest at $K_{s}$ band

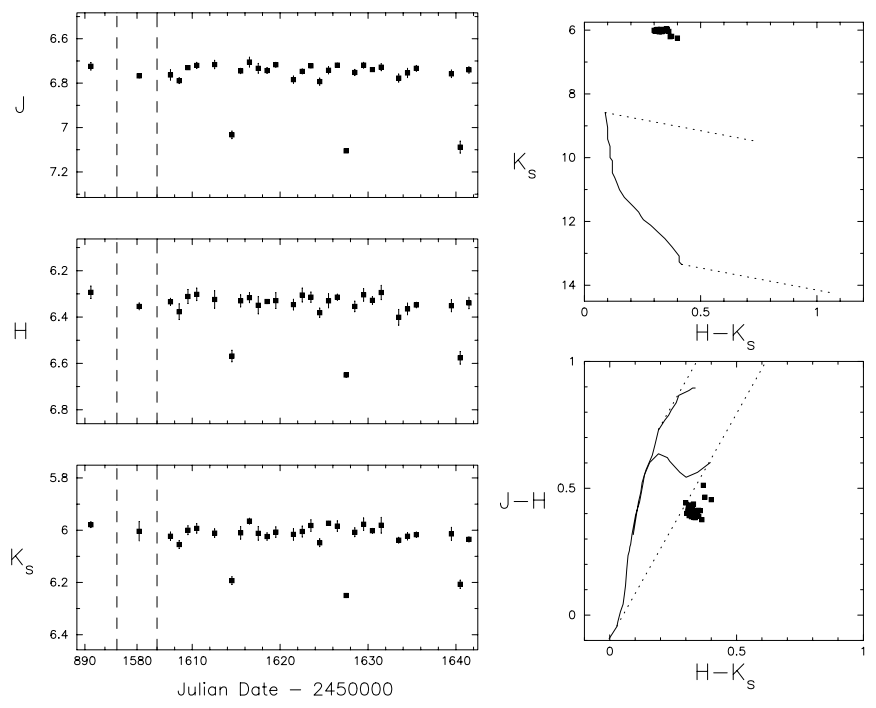

FIG. 6.-Photometric data for star 8783 (also known as BM Ori), classified as an eclipsing system based on the simultaneous drops in the $J, H$, and $K_{s}$ magnitudes on 3 discrete days. This eclipsing system was identified previously from optical observations (see, e.g., Antokhina, Ismailov, \& Cherepashchuk 1989 and references therein). 

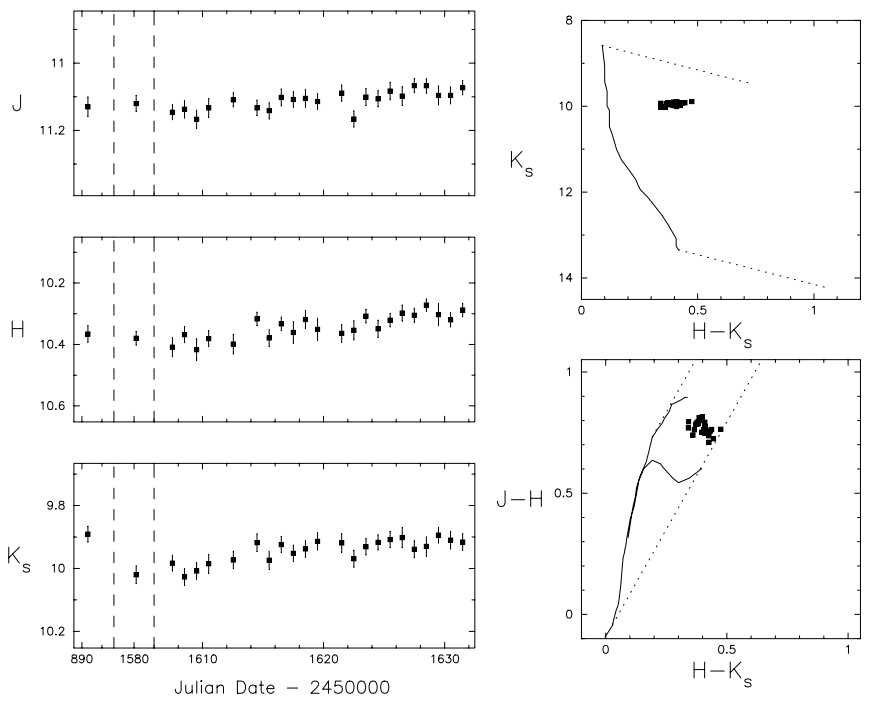

Fig. 7.- Photometric data for star 4067, an example of a star that steadily increased in brightness in the 2000 March-April time period. The 1998 March photometry, though, indicates that this has not been a longterm trend.

and the stellar colors become bluer as the star gets fainter. Figure 9, on the other hand, illustrates one of the more dramatic instances where the amplitude fluctuations are largest at $J$ band and the stellar colors become redder as the star gets fainter, with the fluctuations occurring on a day-to-day basis. Not all stars with color changes vary continuously in time, as Figure 10 presents a star in which the photometry was relatively constant for the first 2 weeks of the 2000 March-April observations prior to the star's becoming fainter by 1-2 mag in each band, with progressively redder colors. Further, as the star faded in brightness,
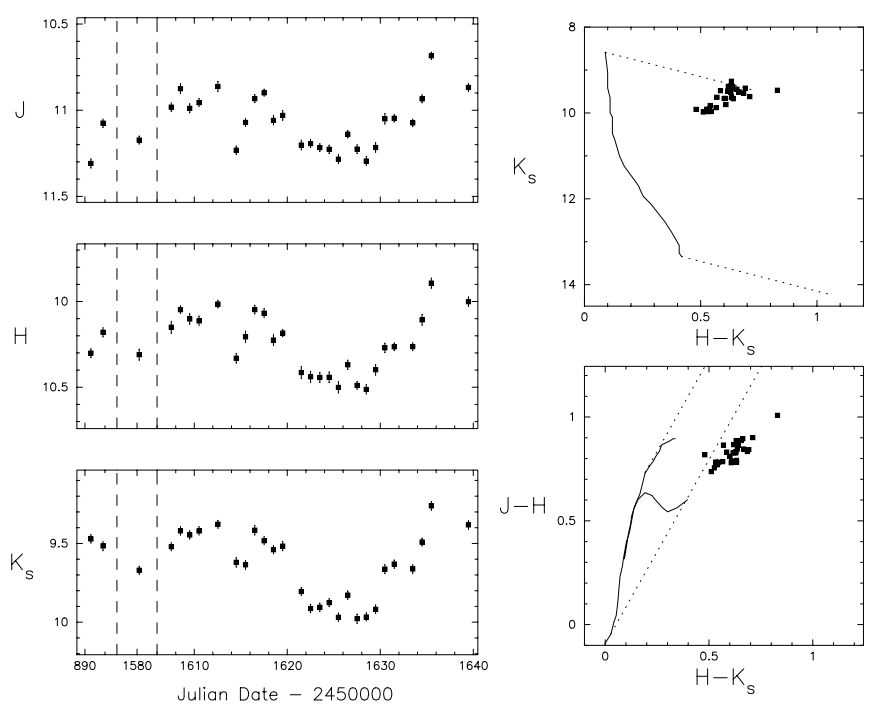

Fig. 8.-Photometric data for star 5707 (also known as YY Ori), an example of a star in which the stellar colors become bluer as the star gets fainter. The senses of the color-magnitude changes are the opposite of that expected from either rotational modulation by hot spots or extinction variations but are consistent with a model in which the geometry or mass accretion rate in a circumstellar disk changes with time.
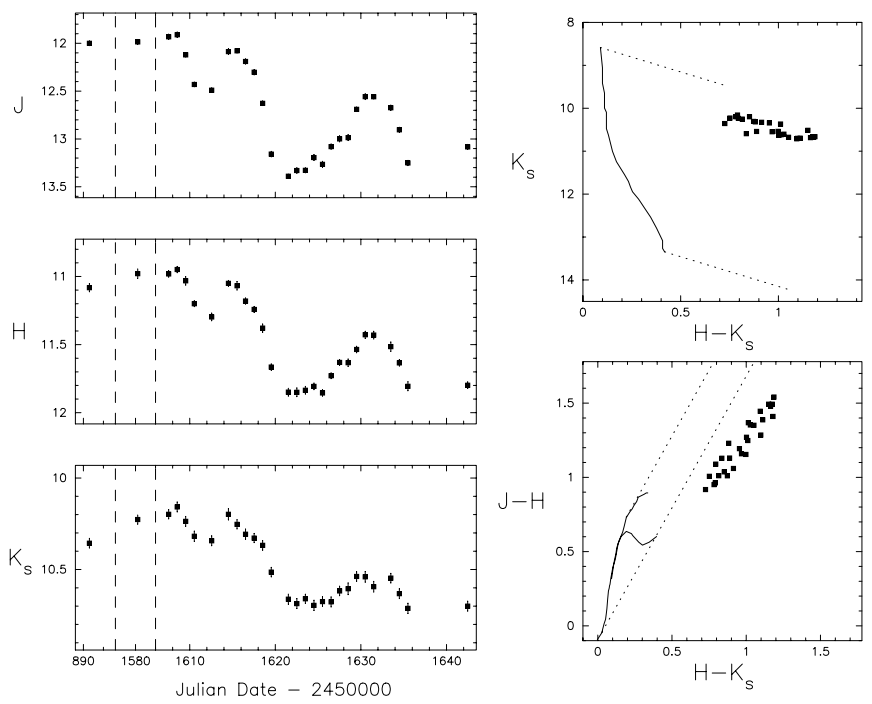

Fig. 9.-Photometric data for star 11926 (also known as AO Ori), an example of a star in which the stellar colors become redder as the star gets fainter. Qualitatively, the photometric fluctuations are consistent with either the presence of time-variable hot spots or variations in the amount of extinction.

a near-infrared excess became apparent for 2-3 days. By the end of the time-series observations, the magnitudes and colors were nearly back to the values at the start of the observations. Figure 11 shows another star with large color variations, but in which the slope of the photometric fluctuations is less steep in the color-color diagram and more steep in the color-magnitude diagram than in the prior examples. The photometric fluctuations in this star are also suggestive of quasi-periodic variations.

Finally, we illustrate two examples of stars that display long-term photometric variability. The star in Figure 12

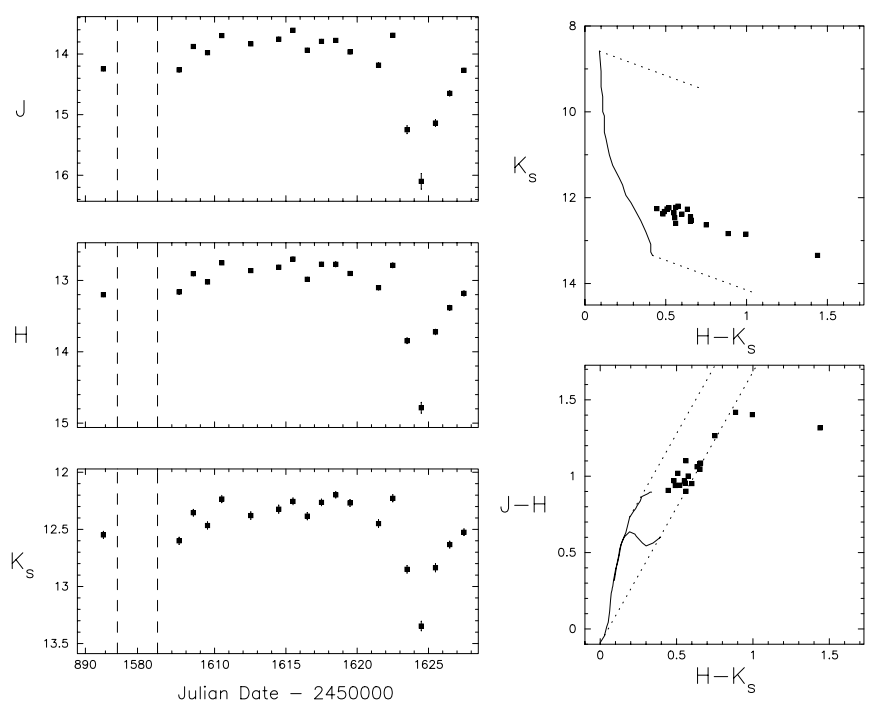

FIG. 10.- Photometric data for star 1048, another example of a star in which the stellar colors become redder as the star gets fainter (see also Fig. 9). In this instance, the stellar magnitudes are relatively constant for the first 2 weeks of the time series before the star becomes fainter by over 1 mag in each band, with progressively redder colors over a period of a couple of days. As the star fades in brightness, a near-infrared excess becomes apparent for 2-3 days. 

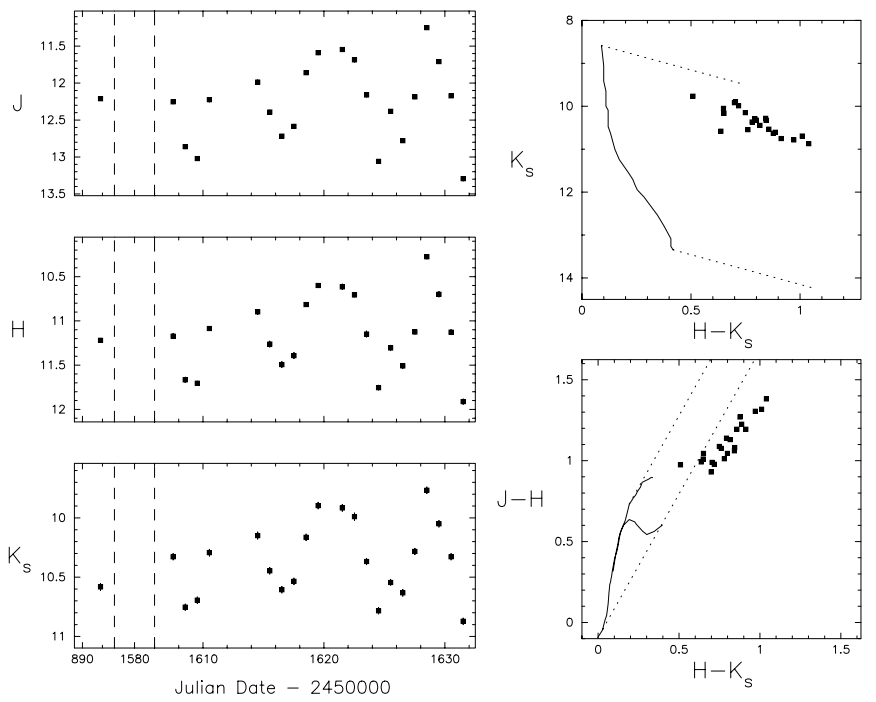

Fig. 11.-Photometric data for star 13688 (also known as AW Ori), another example of a star in which the stellar colors become redder as the star gets fainter (see also Figs. 9 and 10). In this instance, the star exhibits quasi-periodic fluctuations, and the colors and magnitudes vary along a vector that is shallower in the color-color diagram than prior examples and steeper in the color-magnitude diagrams.

was not identified as a variable in the 2000 March-April time-series data, but it decreased in brightness by $\sim 1 \mathrm{mag}$ relative to the 1998 March observations. The star in Figure 13 is variable in the 2000 March-April observations and shows even larger photometric fluctuations when the 1998 March data are included. The long-term variability characteristics are such that while the star became fainter at $J$ band over a 2 year period, it simultaneously brightened at $K_{s}$ band.

\section{PROPERTIES OF THE VARIABLE STARS}

In this section, we discuss the properties of the 1006 uniformly selected variable stars from sample 1 (see Table 3 ),
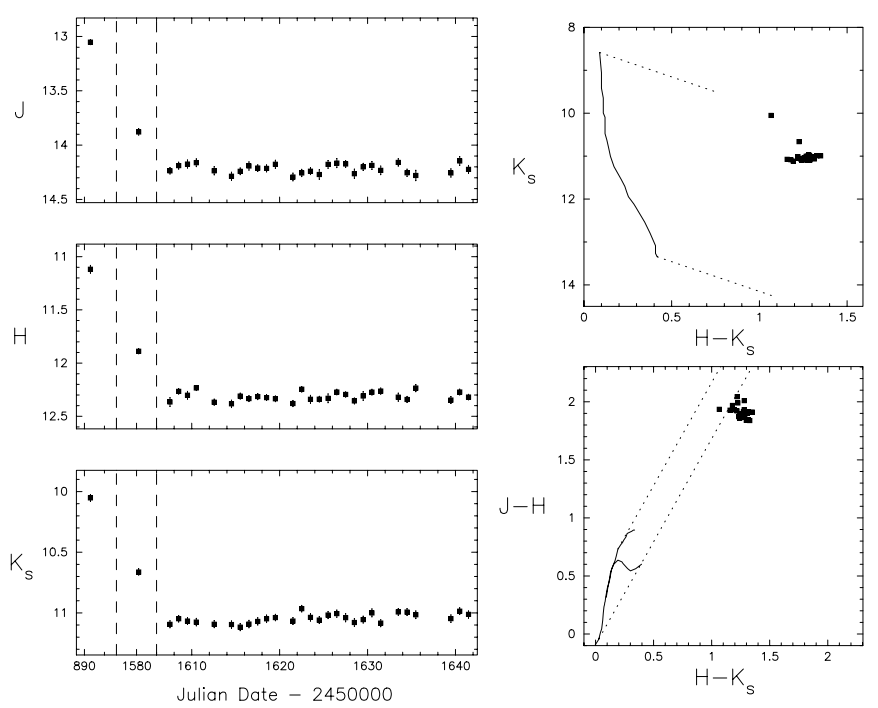

FIG. 12.-Photometric data for star 10527, an example of a star that is not variable in the 2000 March-April time frame but exhibits longer term photometric fluctuations in both the 1998 March and 2000 February observations.
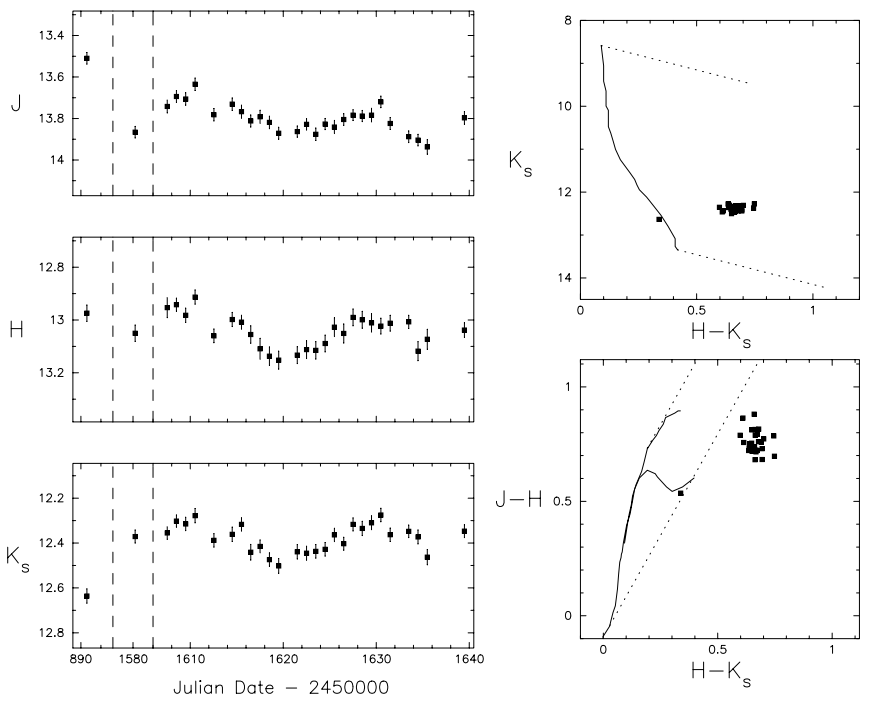

FIG. 13.-Photometric data for star 5841 (also known as JW 101 and V1314 Ori), a second example of a star that exhibits long-term photometric variability relative to the 2000 March-April time-series data (see also Fig. 12). In this case, the star is identified as a variable in the 2000 March-April data but exhibits even larger fluctuations in the 1998 March data. The long-term variability is such that the star became fainter at $J$ band while simultaneously getting brighter at $K_{s}$ band.

including the spatial distribution of near-infrared variables compared with other tracers of the Orion star-forming region, the fraction of the Orion stars exhibiting nearinfrared variability, and the distribution of the variable stars in color-magnitude and color-color diagrams. In the following section, we analyze the amplitude, color, and timescale characteristics of the variable stellar population.

\subsection{Spatial Distribution}

The spatial distribution of the variable stars from sample 1 is presented in Figure 14. Also shown for comparison are (1) the spatial distribution of all stars brighter than $K_{s}=$ 14.8 displayed both as discrete sources and as a surface density map, (2) $\mathrm{H} \alpha$-emitting stars from the Kiso surveys with a Kiso class of 3, 4, or 5 (Wiramihardja et al. 1991, 1993; see also Parsamian \& Chavira 1982), (3) the 305 variable stars from sample 1 that have a near-infrared excess in the $J-H$ versus $H-K_{s}$ color-color diagram (see $\S 4.3$ ), and (4) a map of the Orion molecular cloud as traced by ${ }^{13} \mathrm{CO}$ (1-0) emission (Bally et al. 1987).

Figure 14 shows that near-infrared variable stars are found in substantial numbers between declinations of about $-7^{\circ}$ and -4.5 , which closely reflects the distribution of enhanced $K_{s}$-band star counts. The densest concentration of variables is located toward the Trapezium region of the ONC at $\delta \sim-5.5$ with a secondary density peak near NGC 1977 at $(\alpha, \delta) \sim(83: 8,-4.8)$. Variable stars with a near-infrared excess are distributed over a more restricted declination range than the complete variable star sample and share a similar spatial distribution with the $\mathrm{H} \alpha$ stars, although the Orion Nebula prevents identification of $\mathrm{H} \alpha$ emitting stars in the Trapezium region. The OB stars, not shown in Figure 14, are a sparser population but, nonetheless, are concentrated within the same declination range as the variable and $\mathrm{H} \alpha$-emitting stars (Brown, de Geus, \& de Zeeuw 1994). Further, the spatial distribution of each of 


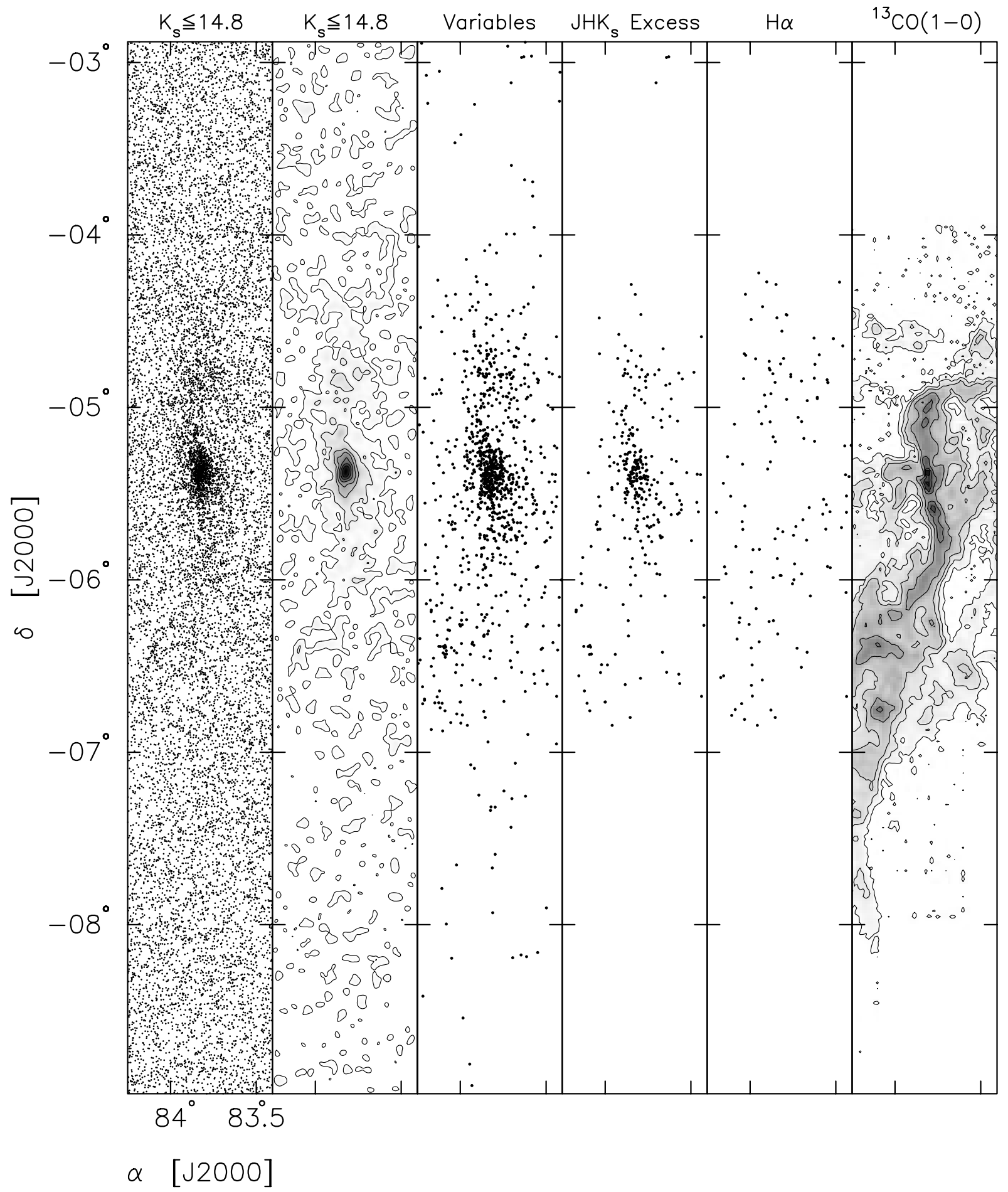

Fig. 14. - Spatial distribution of stars and molecular gas toward the Orion Nebula cluster. Starting with the leftmost panel, these figures show (1) the spatial distribution of stars with $K_{s} \leq 14.8$; (2) the surface density of stars with $K_{s} \leq 14.8$, where the surface density map was created by convolving the stellar spatial distribution shown in the first panel with a Gaussian kernel of size $\sigma=60^{\prime \prime} ;$ (3) the spatial distribution of variable stars from sample 1 (see Table 3 ); (4) the distribution of variable stars shown in the previous panel that have a near-infrared excess in the $J-H$ vs. $H-K_{s}$ color-color diagram; (5) the distribution of $\mathrm{H} \alpha$-emitting stars from the Kiso $\mathrm{H} \alpha$ survey with a Kiso class of 3, 4, or 5 (Wiramihardja et al. 1991, 1993); and (6) a contour map of the integrated ${ }^{13} \mathrm{CO}(1-0)$ emission $\left(\int T_{R} d v\right)$ from Bally et al. (1987). The contours levels are 1, 5, 10, 20, 30, 40, and $50 \mathrm{~K} \mathrm{~km} \mathrm{~s}^{-1}$. These panels indicate that the variable stellar population follows the large-scale spatial distribution of stars in the Orion A molecular cloud as traced by the total $K_{s}$-band star counts and $\mathrm{H} \alpha$-emitting objects.

these stellar populations closely resembles that of the largescale molecular cloud structure. These properties suggest that most of the near-infrared variables are associated with the Orion A molecular cloud.

\subsection{Fraction of Stars Exhibiting Near-Infrared Variability}

We have taken advantage of the large areal coverage of our observations to estimate the spatial extent and statistical membership of the stellar population associated 
TABLE 5

Stellar Population Associated with Orion A

\begin{tabular}{llccc}
\hline \hline $\begin{array}{c}\text { Surface } \\
\text { Density } \\
\left(\operatorname{arcmin}^{-2}\right)\end{array}$ & $\begin{array}{c}\text { Size } \\
(\operatorname{arcmin})\end{array}$ & $N\left(K_{s} \leq 14\right)$ & $N_{\text {var }}$ & $f_{\text {var }}$ \\
\hline $0.25 \ldots \ldots$. & $24 \times 144$ & 2704 & 786 & $0.29 \pm 0.01$ \\
$0.50 \ldots \ldots$. & $20 \times 87$ & 2148 & 627 & $0.29 \pm 0.01$ \\
$0.75 \ldots \ldots$. & $14 \times 86$ & 1881 & 554 & $0.29 \pm 0.01$ \\
$1.00 \ldots \ldots$. & $12 \times 57$ & 1488 & 445 & $0.30 \pm 0.01$ \\
$1.25 \ldots \ldots$. & $12 \times 40$ & 1262 & 386 & $0.31 \pm 0.02$ \\
$2.50 \ldots \ldots$. & $9.3 \times 20$ & 895 & 258 & $0.29 \pm 0.02$ \\
$3.75 \ldots \ldots$. & $7.9 \times 17$ & 752 & 202 & $0.27 \pm 0.02$ \\
$5.00 \ldots \ldots$. & $7.0 \times 14$ & 621 & 158 & $0.25 \pm 0.02$ \\
$7.50 \ldots \ldots$. & $4.9 \times 9.5$ & 397 & 94 & $0.24 \pm 0.03$ \\
$10.00 \ldots \ldots$. & $4.2 \times 6.0$ & 253 & 52 & $0.21 \pm 0.03$ \\
\hline
\end{tabular}

with the northern portion of the Orion A molecular cloud using the $K_{s}$-band star counts, as outlined in the Appendix. The results of this analysis are summarized in Table 5, including various surface densities at which the stellar density enhancement identified in Figure 14 can be defined, the corresponding angular extent, stellar census counts, and the number and fraction of variable stars at these surface density limits. At the lowest defined surface density level ( $\sigma \sigma$ above the mean field star surface density), the density enhancement contains $\sim 2700$ stars with $K_{s} \leq 14$ distributed over a $0.4 \times 2.4(3.4 \mathrm{pc} \times 20 \mathrm{pc})$ region. The $1 \sigma$ boundary encompasses 786 variable stars, or $78 \%$ of the total variable star population. The 220 variable stars not within this boundary tend to be located on the periphery of the density enhancement and may also be cloud members, since the $1 \sigma$ boundary is artificially limited by the field star contamination. If not true cloud members, these variables may be part of the larger scale Orion star-forming region, which extends many tens of parsecs in all directions, although some may be field stars as discussed in the Appendix.

Having quantified the total number of stars in the Orion A molecular cloud detectable to the sensitivity limits of our observations, we can now establish the fraction of the stellar population that is variable. At the lowest surface density at which we can define the density enhancement, $\sim 29 \%$ of the stellar members are variable within the photometric noise limits of the data (see Table 5). This fraction does not change appreciably with the surface density used to define the density enhancement. While there is some suggestion that the variable fraction decreases in the inner $5^{\prime} \times 5^{\prime}$ near the Trapezium, it is more difficult to identify variable stars in this region because of the bright sky background from the Orion Nebula, which increases the photometric noise.

To investigate the variable star fraction as a function of magnitude, Figure 15 shows $K_{s}$-band histograms for the cloud population and for the variable stars within the $1 \sigma$ boundary. The field stars have been subtracted from the cloud population using a procedure similar to that described in the Appendix, but applied to the differential magnitude intervals. The $K_{s}$-band histogram for the cloud population as a whole is broadly peaked at $\sim 11-13 \mathrm{mag}$ with a median value of $11.7 \mathrm{mag}$, which is significantly brighter than the completeness limit of the observations $\left(K_{s}=14.8\right)$. Similarly, the $K_{s}$-band histogram for the vari-

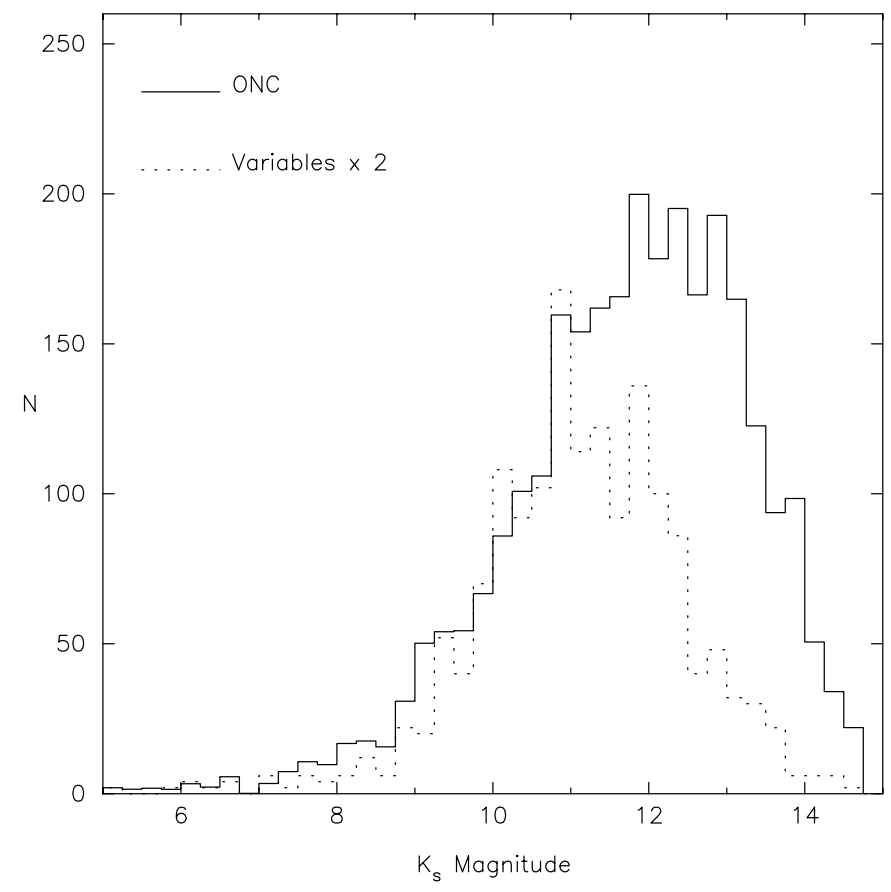

Fig. 15.-Histogram of the $K_{s}$-band magnitudes for stars in the Orion A molecular cloud and the variable star population. The field star contribution to the cloud population has been subtracted from the observed star counts using the procedure described in the Appendix applied to differential magnitude intervals. The completeness limit of the observations is $K_{s}=14.8$. This figure indicates that the variable star population identified with these observations tends to be the brighter stars in the cluster. The lack of faint variable stars is likely a result of increased photometric noise at these magnitudes that masks any low-amplitude photometric fluctuations.

able star population peaks well above the completeness limit, but at a median magnitude (11.1 mag) that is brighter than the cloud population. Further, $\sim 45 \%$ of the cloud members brighter than $K_{s}=11$ show near-infrared variability, but only $14 \%$ of stars fainter than $K_{s}=12$ are nearinfrared variable within the noise limits of the data.

To determine whether the fainter cloud members are intrinsically less variable or whether the increased photometric noise (see Fig. 2) is masking low-amplitude fluctuations, a Monte Carlo simulation was run in which simulated variability common to all three bands was added to random noise. The amplitude of the noise in each band was set based on the photometric noise in the actual data. The variability was simulated with a Gaussian random number generated with a dispersion ranging from 0.01 to $0.2 \mathrm{mag}$. Variable stars in the simulated photometry were then identified using a Stetson index threshold of 0.55 . For $K_{s}=12$, roughly one-half of the simulated stars are identified as variable when the dispersion in the photometric fluctuations is $0.03 \mathrm{mag}$. Since the median $K_{\mathrm{s}}$-band amplitude dispersion for identified variables over all magnitudes is $\sim 0.05$ (see § 5.1), these results suggest that the lack of variable stars at faint magnitudes is indeed a result of increased photometric noise.

\subsection{Mean Colors and Magnitudes}

Basic physical properties of the variable star population can be constrained by the observed stellar magnitudes and colors. As already shown in Figure 15, the histogram of the 
$K_{s}$-band magnitudes for the variable star population peaks slightly brighter than the peak for the cloud population. The overall peak has been seen in several previous studies of the Trapezium region of the ONC (Zinnecker, McCaughrean, \& Wilking 1993; McCaughrean et al. 1995; Lada, Alves, \& Lada 1996; Simon, Close, \& Beck 1999; Hillenbrand \& Carpenter 2000) and can be accounted for by a combination of pre-main-sequence evolution for a $\sim 1 \mathrm{Myr}$ cluster and a Miller-Scalo initial mass function (Zinnecker et al. 1993). Assuming this age and neglecting extinction and near-infrared excesses, the peak in the variable star $K_{s}$-band histogram corresponds to a stellar mass of $\sim 0.2-0.6 M_{\odot}$, according to the evolutionary theory of D'Antona \& Mazzitelli (1997), for a cluster distance of $480 \mathrm{pc}$ (Genzel et al. 1981). Extensive photometric and spectroscopic obser- vations have shown that the ONC stellar population near the Trapezium does in fact consist predominantly of young ( $\leqslant 1$ Myr), low-mass stars (Herbig \& Terndrup 1986; Hillenbrand 1997). Since the peak in the $K_{s}$-band histogram is a feature of the entire stellar density enhancement and not just of the area near the Trapezium, this result suggests that the majority of the cloud population, and hence the variable stars, is relatively young and of low mass.

The $K_{s}$ versus $H-K_{s}$ color-magnitude diagram shown in Figure 16 further supports the conclusion that most of the variable stars are young $(\lesssim 1 \mathrm{Myr})$, low-mass $\left(\lesssim 1 M_{\odot}\right)$, moderately reddened members of the Orion A molecular cloud. The color scale in this figure represents all detected stars, $\sim 85 \%$ of which are likely field stars (see Appendix). The nonvariable stars (predominantly field stars) are

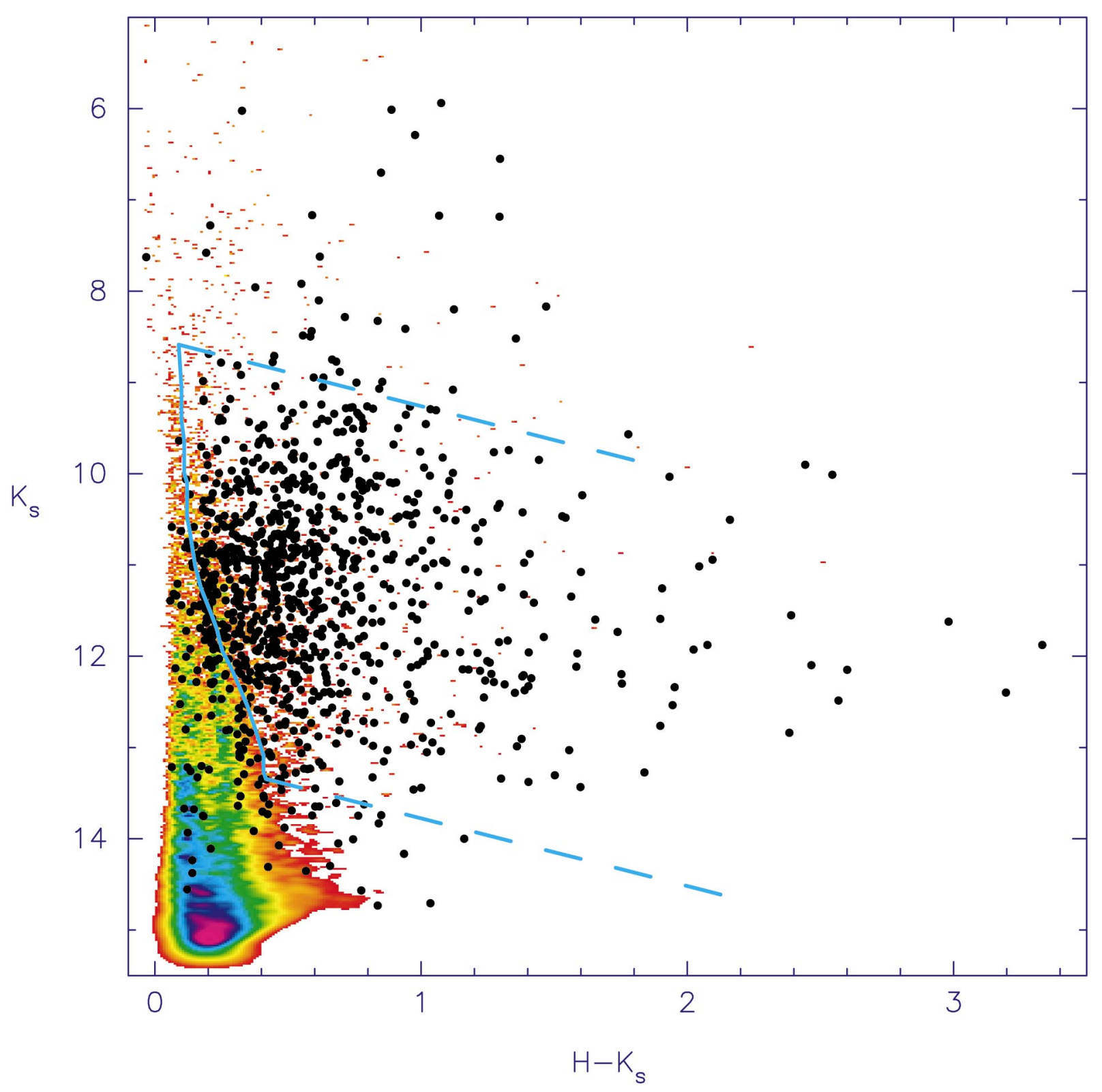

FIG. 16. $-K_{s}$ vs. $H-K_{s}$ color-magnitude diagram for all stars (color scale) and the variable stars (circles) identified from the 16 nights in which all tiles were observed. For reference, the solid blue curve shows the 1 Myr pre-main-sequence isochrone from D'Antona \& Mazzitelli (1997) for stellar masses between 0.08 and $3.0 M_{\odot}$. The dashed lines indicate the reddening vector for 10 mag of visual extinction from Cohen et al. (1981), transformed into the 2MASS photometric system (Carpenter 2001). The lowest halftone is $1 \%$ of the peak density. This figure shows that the observed magnitudes and colors for the majority of the variable population are consistent with reddened pre-main-sequence stars with masses $\lesssim 3 M_{\odot}$. 


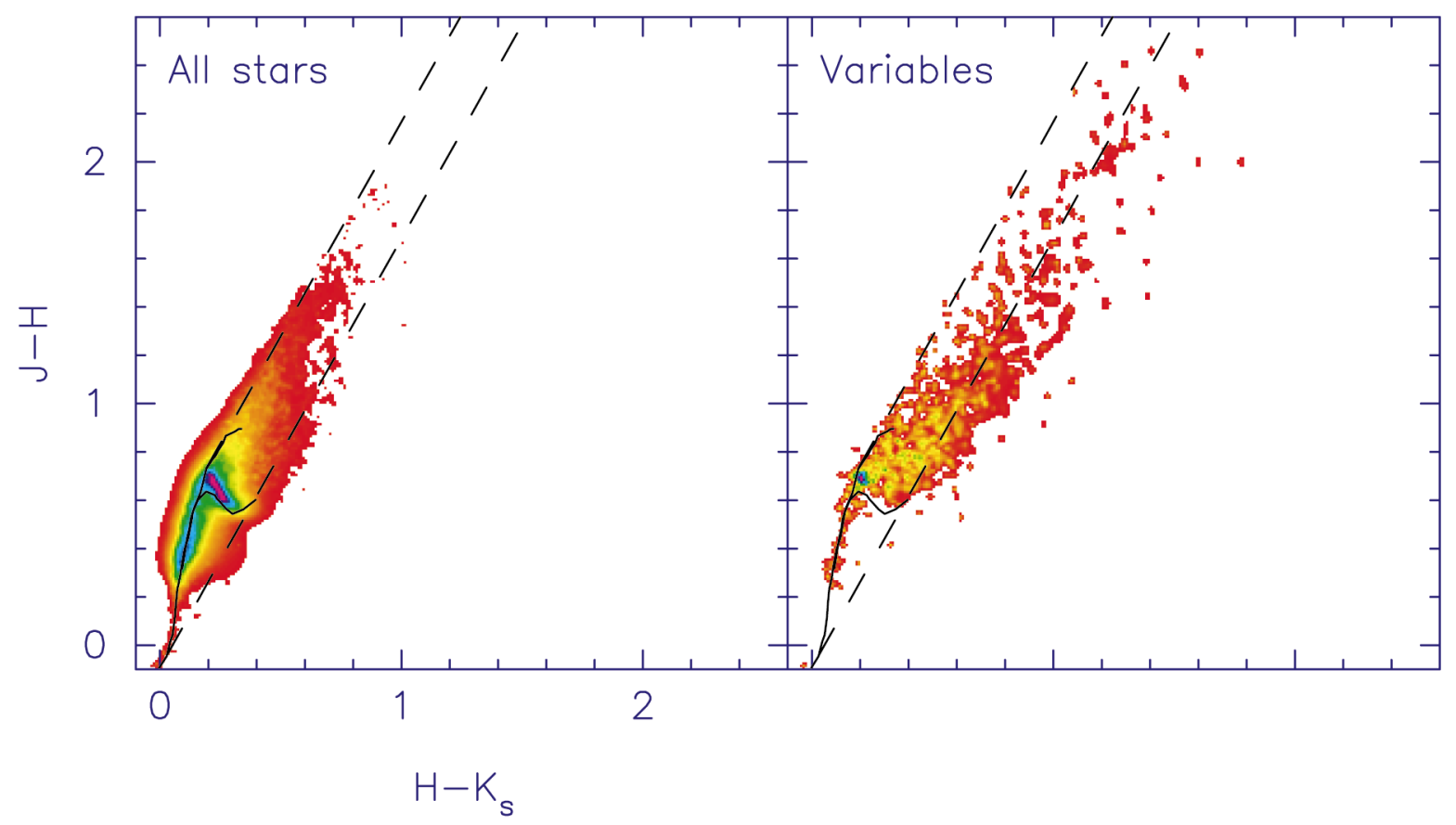

FIG. 17. - The $J-H$ vs. $H-K_{s}$ color-color diagrams for all stars (left) and the variable stars (right) identified from the 16 nights in which all tiles were observed. The lowest halftone in each panel begins at $1 \%$ of the peak density. The stars represented in the left panel are dominated by field stars unrelated to the Orion A molecular cloud. The variable stars are on average redder than the field star population, and $\sim 30 \%$ have colors indicating the presence of a near-infrared excess.

located primarily to the left of the $1 \mathrm{Myr}$ isochrone, while most of the variable stars (circles) are located to the right and are consistent with reddened pre-main-sequence objects.

To further examine the properties of the variable star population, Figure 17 shows the $J-H$ versus $H-K_{s}$ colorcolor diagram for all stars in our sample (left) compared with the variable stellar population (right). This figure shows that the majority of nonvariables have colors consistent with unreddened main-sequence stars either in front of the cloud or at a variety of distances but along lines of sight exterior to the cloud boundaries, or reddened mainsequence and giant stars seen through the cloud. The variable stars are systematically redder compared with the nonvariable stars, many with colors that place them in a region of the diagram that cannot be explained by interstellar extinction. These near-infrared colors are characteristic of young, low-mass classical T Tauri stars (CTTSs) surrounded by optically thick accretion disks (Lada \& Adams 1992; Meyer, Calvet, \& Hillenbrand 1997) and moderately reddened. Approximately $30 \%$ of the variable stars have near-infrared colors consistent with CTTSs as identified in the $J-H$ versus $H-K_{s}$ color-color diagram, suggesting that up to $50 \%$ of the variable star population may be CTTSs if the efficiency factor for this diagram, as described in the appendix of Hillenbrand et al. (1998), is adopted. Many of the variable stars, however, do not have such distinctive near-infrared colors. The variable stars without substantial near-infrared excesses are either CTTSs with small excesses or weak-lined T Tauri stars (WTTSs), which as a class do not have strong signatures of optically thick circumstellar disks. Near-infrared variability therefore appears to be a characteristic of both CTTSs and WTTSs.

\section{CHARACTERISTICS OF THE VARIABILITY}

After establishing that most of the near-infrared variable stars are young, low-mass stars associated with the Orion A molecular cloud, we now investigate in more detail the variability characteristics exhibited by these stars. Our goal is to use the statistics inherent in our large sample to categorize the predominant types of near-infrared photometric fluctuations. To include as complete a sample as possible in characterizing the photometric variations, we incorporate all of the 2000 March-April time-series data for the stars listed in Table 4 (unless otherwise stated). Strictly speaking, this results in nonuniform time coverage for stars as a function of their location in the survey region and also incorporates some stars that were included as variables based on somewhat subjective criteria. In practice, however, $\sim 77 \%$ of the variables are located in the center tiles 3-5 (see Table 2 ) and were observed on at least 25 of the possible 29 nights. Further, the number of stars included in the variable star list for reasons other than high Stetson index amount to less than $10 \%$ of the entire sample. Therefore, the following results should closely reflect those that would be obtained from a more uniformly selected sample.

\subsection{Amplitude of Magnitude and Color Changes}

The amplitude of the variability was characterized by computing the observed rms and peak-to-peak fluctuations in the magnitudes and the colors for individual stars. Since the observed photometric fluctuations reflect both actual astrophysical variability and photometric noise, a statistical correction needs to be applied to recover the intrinsic amplitude. For the rms values, the actual variability amplitude $\left(\sigma_{\mathrm{var}}\right)$ was estimated by subtracting in quadrature the 
expected rms due to photometric noise ( $\sigma_{\text {noise }} ;$ see eq. [3]) from the observed rms $\left(\sigma_{\text {obs }} ;\right.$ eq. [2]) as

$$
\sigma_{\mathrm{var}}=\sqrt{\sigma_{\mathrm{obs}}^{2}-\sigma_{\text {noise }}^{2}} \text {. }
$$

The noise correction for the peak-to-peak amplitudes is not as straightforward, since it depends on the noise in the individual observations (which varies in the time-series data) and the number of samples. A Monte Carlo simulation was run 500 times for each star to estimate the average expected peak-to-peak amplitude due to noise, given the number of measurements and the photometric uncertainties. This expected peak-to-peak amplitude was subtracted linearly from the observed value to estimate the intrinsic peak-topeak fluctuations.

Histograms of the peak-to-peak and rms amplitudes for the magnitudes and colors, after correcting the observed values for the photometric noise, are shown in Figures 18 and 19. The top panels in each figure show the histograms over the full dynamic range of the amplitudes, and the bottom panels emphasize the distribution at low ampli- tudes, where most of the variable stars in fact reside. Statistics on the maximum, mean, median, and dispersion in the amplitudes are summarized in Table 6. The peak-topeak fluctuations in the magnitudes are a couple tenths of a magnitude on average but can be as large as $2.3 \mathrm{mag}$ at $J$ band and $1.2 \mathrm{mag}$ at $K_{s}$ band. Peak-to-peak fluctuations as large as $1.7 \mathrm{mag}$ at $K_{s}$ band are observed in three stars when the 1998 March data are included. Fluctuations in the colors are less pronounced, as most of the photometric variations are essentially colorless within the photometric noise of the data. Thus, stars that exhibit large magnitude and color variations (see, e.g., Figs. 8-11) are among the more extreme cases of near-infrared variability in our sample.

\subsection{Correlation of Magnitude and Color Changes}

For stars with significant color variations, any correlation between the color-magnitude and color-color changes can serve as a clue to the origin of the variability. Cases of stars becoming bluer as they fade (e.g., Fig. 8) and
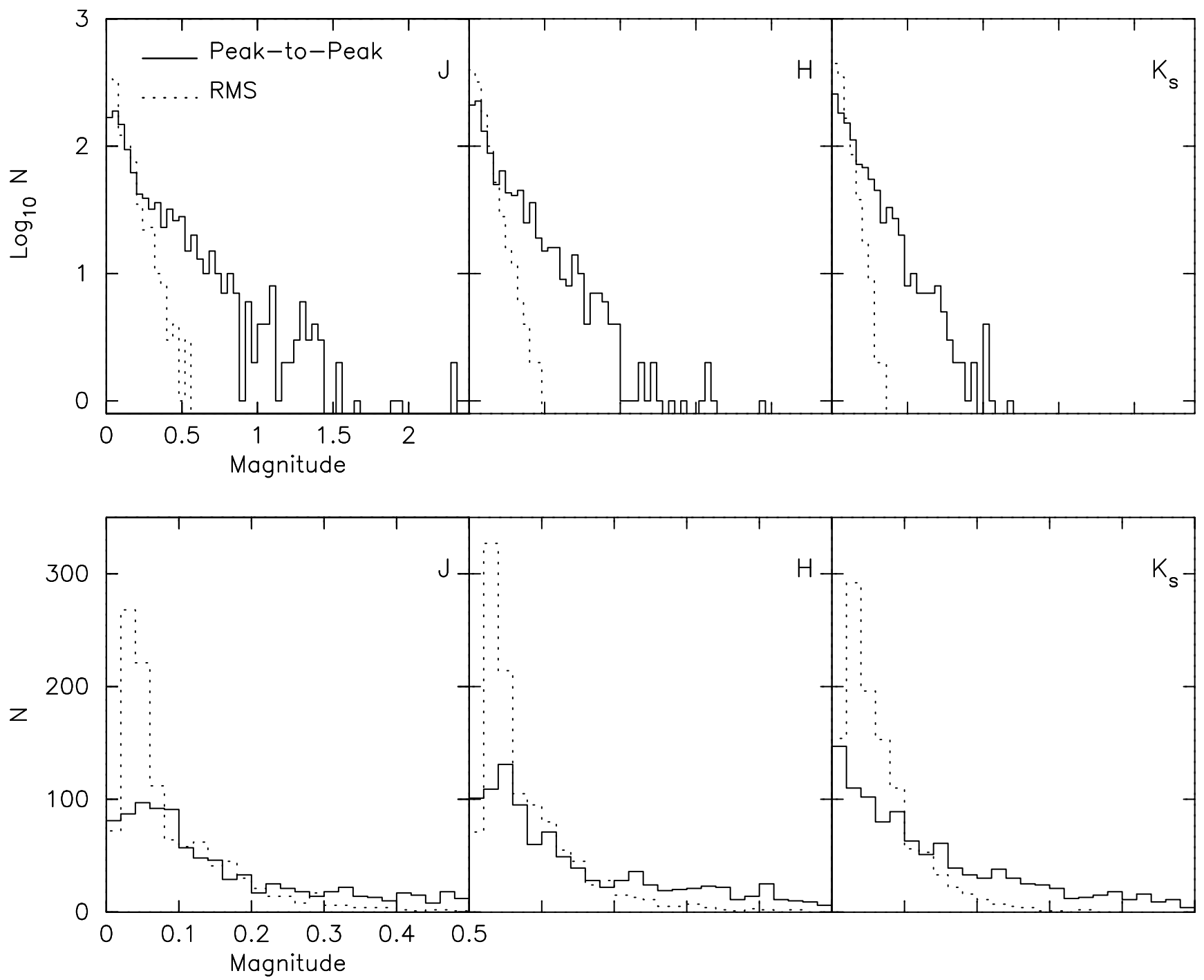

FIG. 18.- Histograms of the variable star peak-to-peak (solid histograms) and rms (dotted histograms) amplitudes in the $J, H$, and $K_{s}$ data after correcting the observed amplitudes for photometric noise (see $\S 5.1$ ). The top panels show histograms over the full dynamic range, and the bottom panels show in more detail the distributions at low amplitudes, which contain most of the variables. The amplitudes were computed using all measurements in the 2000 March-April time series. 

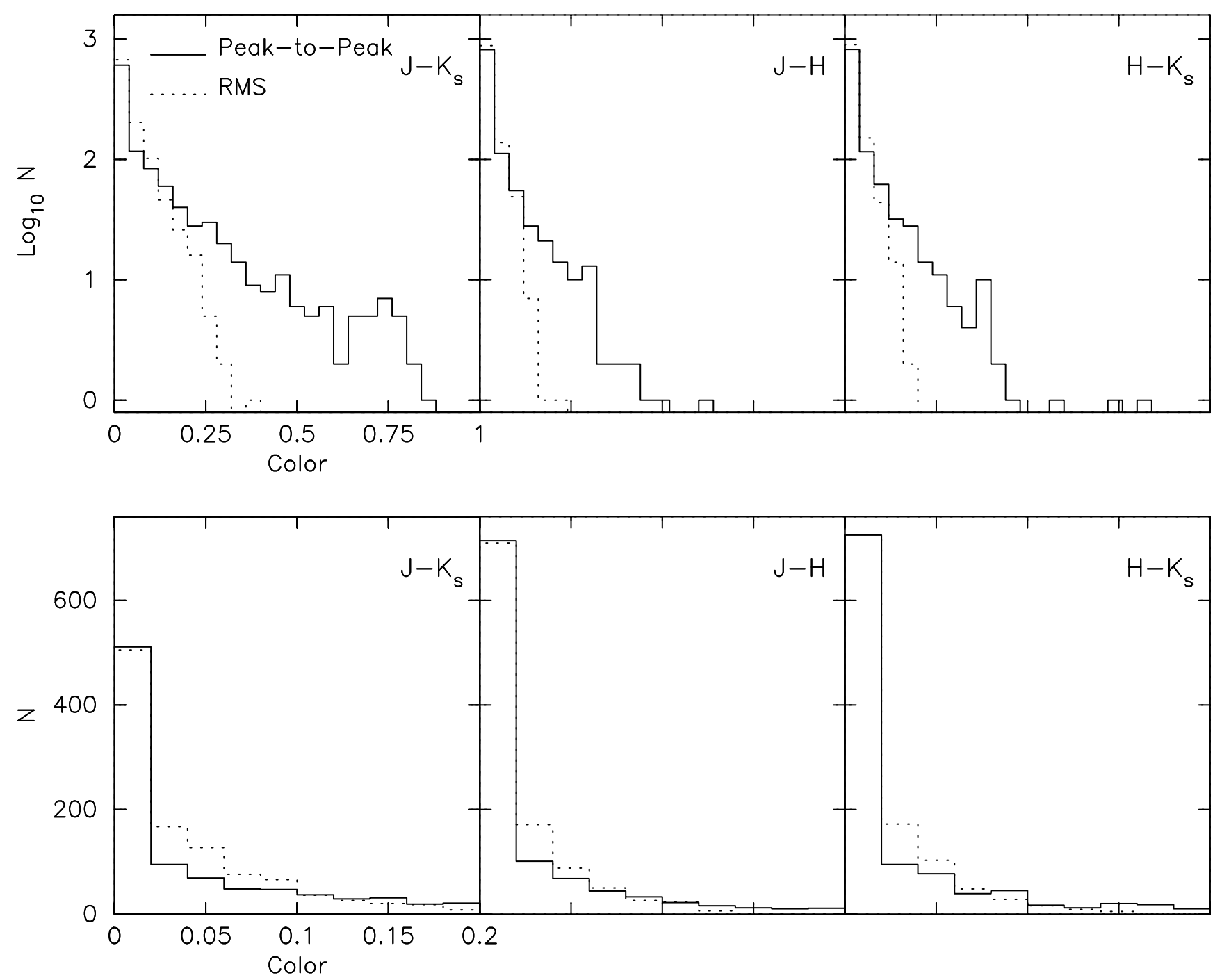

FIG. 19. - Same as Fig. 18, but for the $J-K_{s}, J-H$, and $H-K_{s}$ colors

others becoming redder as they fade (e.g., Figs. 9-11) exist in our data, with the photometric changes within a star usually well correlated along narrow vectors in the various color-color and color-magnitude diagrams. However, not all possible vector orientations are found in the time-series data. To quantify the observed correlations, the slopes of the photometric variations in the $J$ versus $J-H, K_{s}$ versus $H-K_{s}$, and $J-H$ versus $H-K_{s}$ diagrams were computed for individual stars. For each correlation, only stars in which the observed rms in the colors exceeded the expected photometric uncertainties by $50 \%$ were included, so that the derived slopes would not be dominated by noise in the data. As only $\sim 10 \%$ of the variable stars satisfied this criterion, the analysis that follows is most appropriate for those objects with relatively large-amplitude color variations and may not be applicable to those with largely colorless variability. The routine FITEXY (Press et al. 1992), which incorporates uncertainties in both axes in computing

TABLE 6

VARIAble Star Amplitudes IN 2000 March-APRIL

\begin{tabular}{|c|c|c|c|c|c|c|c|c|}
\hline \multirow[b]{2}{*}{ BAND } & \multicolumn{4}{|c|}{ PeaK-to-Peak (mag) } & \multicolumn{4}{|c|}{ RMS (mag) } \\
\hline & Maximum & Mean & Median & Dispersion & Maximum & Mean & Median & Dispersion \\
\hline ........ & 2.31 & 0.26 & 0.15 & 0.31 & 0.54 & 0.09 & 0.06 & 0.09 \\
\hline$H \ldots \ldots \ldots \ldots$ & 1.95 & 0.22 & 0.12 & 0.25 & 0.45 & 0.08 & 0.05 & 0.07 \\
\hline$K_{s} \ldots \ldots \ldots \ldots$ & 1.16 & 0.17 & 0.11 & 0.18 & 0.36 & 0.07 & 0.05 & 0.05 \\
\hline$J-K_{s} \ldots \ldots$ & 1.22 & 0.10 & 0.02 & 0.16 & 0.38 & 0.04 & 0.02 & 0.05 \\
\hline$J-H \ldots \ldots$ & 0.61 & 0.03 & 0.00 & 0.07 & 0.21 & 0.02 & 0.00 & 0.03 \\
\hline$H-K_{s} \ldots \ldots$ & 0.82 & 0.04 & 0.02 & 0.08 & 0.19 & 0.02 & 0.00 & 0.03 \\
\hline
\end{tabular}




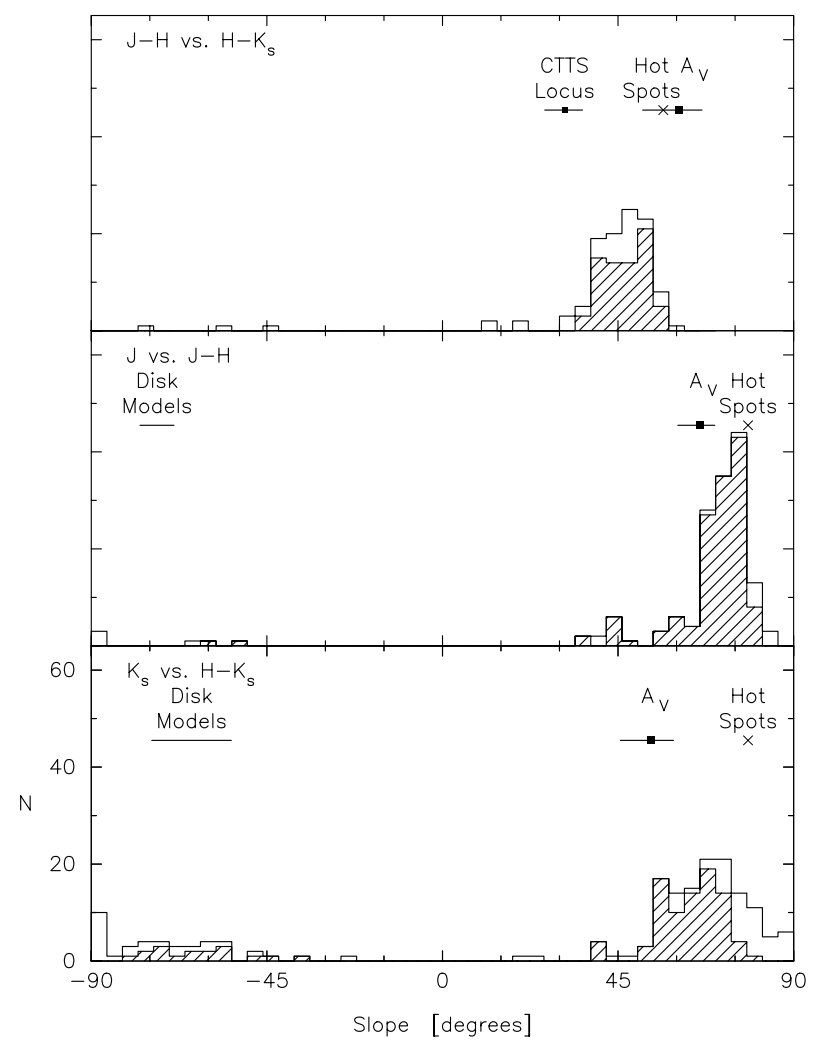

FIG. 20.- - Histograms of the derived slopes in the $J-H$ vs. $H-K_{s}, J$ vs. $J-H$, and $K_{s}$ vs. $H-K_{s}$ diagrams. Only variable stars in which the observed rms of the appropriate colors or magnitudes exceeded the expected rms by a factor of 1.5 are shown. The open histograms are for all stars that meet these criteria, and the hatched histograms are those stars in which the slope have been determined to an accuracy of better than $20 \%$. The predicted slopes based on hot-spot models, extinction variations, and circumstellar disk models (see $\S 6$ ) are indicated. The predicted slopes from cool-spot models are not shown, since they cannot account for the amplitude of the color variability observed in most of these objects. This figure shows that while each of these models can account for some aspect of the variability among these stars, none alone can account for all of the observed trends. the best-fit linear model to the data, was used to derive the slopes. A slope angle of $0^{\circ}$ is defined as positive color change along the $X$-axis with no magnitude or color change along the $Y$-axis. The slope angle increases counterclockwise in the color-color diagram and clockwise in the color-magnitude diagram (since magnitudes are plotted with decreasing values toward the top).

Histograms of the derived slopes in various colormagnitude and color-color diagrams are shown in Figure 20 . The open histograms represent all stars for which slopes were derived. The hatched histograms are stars where the slopes have been determined to better than $20 \%$ accuracy, with a typical $1 \sigma$ uncertainty of $\lesssim 5^{\circ}$. In the $J-H$ versus $H-K_{s}$ diagram, all but five of the stars have positive slopes between $\sim 30^{\circ}$ and $60^{\circ}$. The photometric correlations for the five stars with negative slopes do not vary along a welldefined vector in the color-color diagram, and the slope is not meaningful. Thus, the dominant type of photometric variability in the near-infrared color-color diagram has both colors becoming redder together. In the $J$ versus $J-H$ diagram, the predominant trend is that stars become fainter as the colors get redder, with a slope between $\sim 50^{\circ}$ and $80^{\circ}$. Two stars have negative slopes with uncertainties less than $20 \%$; both are long-term variables with large magnitude and color variations revealed by including the 1998 March data. In the $K_{s}$ versus $H-K_{s}$ diagram, the derived slopes show two distinct trends. In addition to stars with colors becoming redder as they fade (positive slopes; e.g., Figs. 9-11), a number of variables have colors that become bluer as the stars become fainter (negative slopes; e.g., Fig. 8); $\sim 1 \%$ of the total number of variables are of this kind.

Figure 21 compares $J-H$ versus $H-K_{s}$ color-color diagrams for stars with positive and negative slope in the $K_{s}$ versus $H-K_{s}$ color-magnitude diagram. (This figure also includes a panel for periodic stars, which are discussed in $\S$ 5.3.2.) The majority ( $\sim 90 \%)$ of variable stars do not have large color variations and do not appear in this figure. On average, the stars with large color variations tend to have redder near-infrared colors compared with the variable star

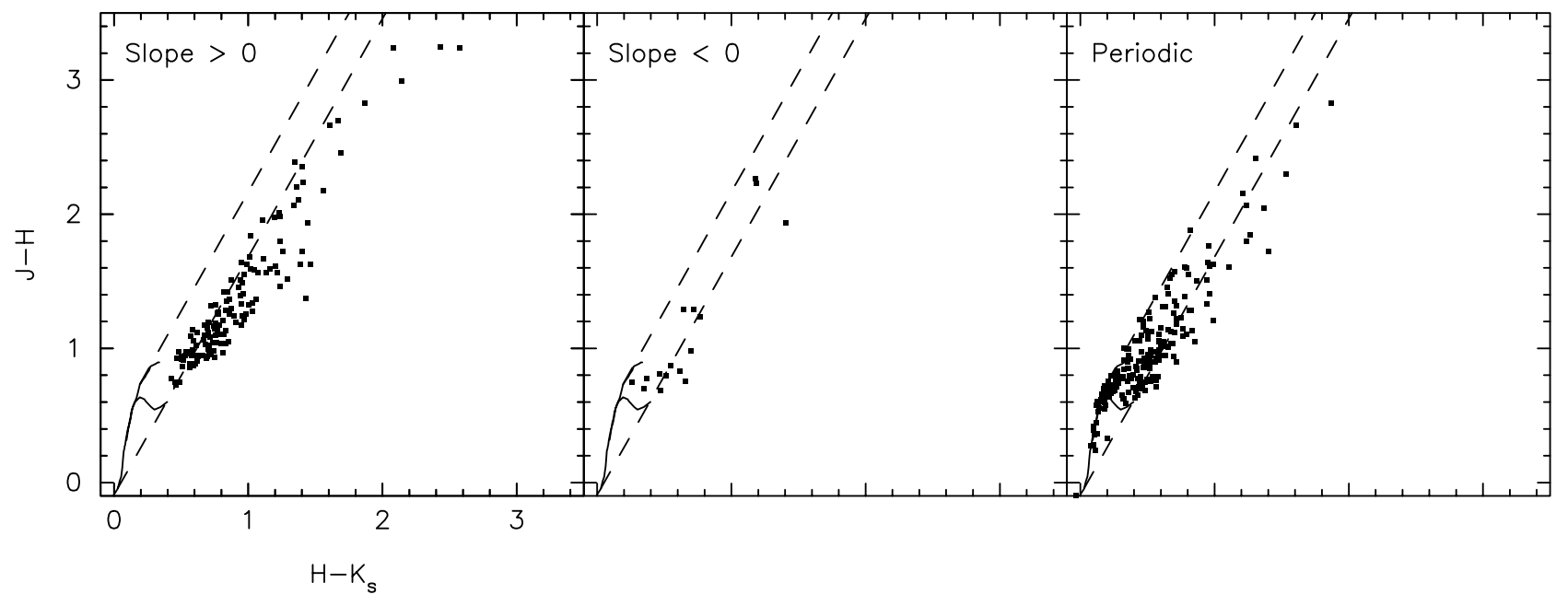

FIG. 21.-The $J-H$ vs. $H-K_{s}$ diagrams for three groups of variable stars. Left: Variable stars that have significant fluctuations in both the observed $K_{s}$ magnitudes and $H-K_{s}$ colors, and photometric fluctuations such that the colors become redder as the star gets fainter (i.e., slope in the $K_{s}$ vs. $H-K_{s}$ diagram is positive). Middle: Stars that have negative slopes such that the colors become bluer as the star gets fainter. Right: Stars that are identified as periodic variables in the near-infrared. Stars with significant color and magnitude variations are generally redder than the periodic stars, and in particular, stars with positive slope variations tend to have near-infrared excesses more so than periodic variables. 
population as a whole (see Fig. 17). Further, $\sim 76 \%$ of the stars with positive slope variations have near-infrared excesses characteristic of CTTSs, compared with $\sim 53 \%$ of the small number of stars with negative slope variations and $30 \%$ for the uniformly selected variable star population of $\sim 1000$ stars $(\S 4.3)$.

\subsection{Temporal Properties \\ 5.3.1. Timescales for Variability}

The temporal variability characteristics can be evaluated on time periods of 1 day to $\sim 1$ month using the 2000 March-April observations, on $\sim 2$ month timescales by incorporating the 2000 February data, and on $\sim 2$ year timescales with addition of the 1998 March data. The temporal properties of the 2000 March-April observations were evaluated using the autocorrelation function (ACF), which measures the similarity of photometric measurements over different time samples. While photometry is nominally available in our data set on a daily basis, the time series can contain gaps of up to 4 days. To account for this nonuniform sampling, the Fourier transform of the observed measurements was computed using the Scargle (1989) algorithm for unevenly sampled data. The power spectrum was then computed from the Fourier transform, and the inverse transform of the power spectra yielded the ACF. The resulting ACF was normalized by the ACF of the sampling function for each star (Scargle 1989) and was sampled at 1 day intervals in accordance with the nominal separation between our observations. A positive value of the ACF at a given time indicates that the photometry is correlated on that timescale, while negative values indicate the photometry is uncorrelated. The timescale of the variability can then be characterized by computing the largest time lag before the ACF first becomes negative. Since the mean value of the photometry was subtracted from the data before computing the ACF, the time lag is a crude measure of the number of consecutive days a star remains brighter or fainter than the mean magnitude over the time series. The longest variability timescale that can be estimated from the data then is approximately half the total time period of the observations. The maximum timescale will vary between 9 and 18 days depending on the spatial location of the star. For variable stars located in the center three tiles $(\sim 77 \%$ of the total number of variable stars), the maximum variability timescale that can be inferred is $\sim 14$ days.

For random noise with an infinite number of samples, the distribution of time lags is a $\delta$-function at $\Delta t=0$; a finite number of samples, though, broadens the ACF. To estimate the expected distribution of time lags for random noise, the ACF was computed for each star as described above but replacing the observed magnitudes by a random number with a Gaussian probability distribution that has a dispersion given by the photometric uncertainties. The time sampling in these simulated data is identical to that in the real observations.

Figure 22 shows the inferred time lags in the three bands for all identified variables (solid lines) and the simulated $J$-band data (dotted line). The $H$ - and $K_{s}$-band simulated data are similar to the $J$-band simulation and for clarity are not shown. This figure demonstrates that the random-noise data have a peak near zero time lag, as expected since each data point is independent. By contrast, the distribution of time lags for the variable stars peaks at 1 day and has a

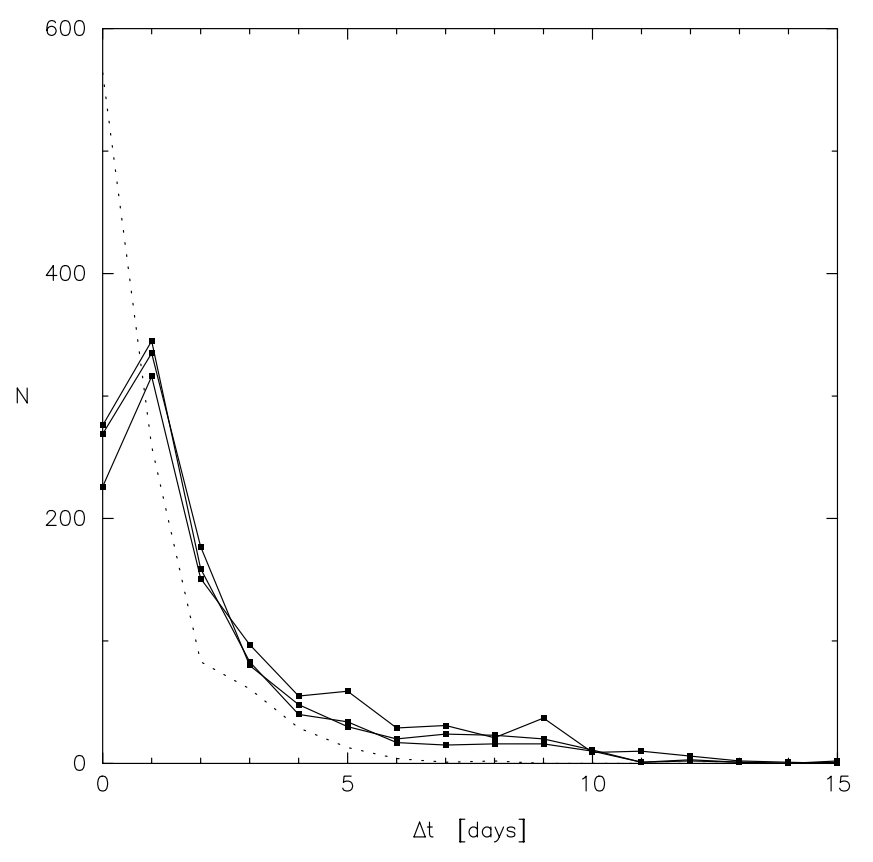

FIG. 22.-Distribution of time lags inferred from the autocorrelation function for the variable stars in the 2000 March-April time-series data. The three solid lines represent the time lags for the $J, H$, and $K_{s}$ data. The dotted line shows the time lags for a simulated data set with the same random noise characteristics and time sampling as the observations. The maximum time lag possible in this ACF analysis is approximately half the time period of the observations. For $77 \%$ of the variable stars, the maximum possible time lag is $\sim 14$ days. This figure shows that in the $\sim 1$ month time-series observations, most of the variability occurs on timescales of a few days, although it can be as long as $10-15$ days in the more extreme cases.

more extended tail toward larger time lags than the simulated observations. The shape of these distributions suggests then that most of the variability occurs on timescales of less than a few days.

The near-infrared variability characteristics on $\sim 2$ month and 2 year timescales were assessed using the 2000 February and 1998 March data (note that the 2000 February data are available only for sources north of $\delta=-6^{\circ}$ ). A long-term variable was identified as a star in which the $J$, $H$, or $K_{s}$ magnitudes from these earlier measurements differed from the average 2000 March-April data by more than 4 times the observed rms scatter in that time period. A $4 \sigma$ deviation was chosen since only about one star in the entire source list should exhibit a fluctuation that large due to random noise. Given typical photometric uncertainties for the variable stars of $0.02-0.03 \mathrm{mag}$, the $4 \sigma$ criterion imposes a minimum amplitude change of $\sim 0.1 \mathrm{mag}$. By this definition, a total of 14 stars exhibited variability in the 2000 February data, but only five of these were variables not previously identified based on the Stetson index in sample 1 or sample 2 (see Table 3). A total of 72 stars exhibited significant photometric fluctuations on $\sim 2$ year timescales, with 26 of these new variables not previously identified from the 2000 March-April data based on the Stetson statistic. The fact that few new long-term variables were identified indicates that most of the short-term variations are not part of larger amplitude variations occurring over long time periods. 


\subsubsection{Periodic Behavior}

The properties of variable stars can be further characterized by determining whether the photometric variations are typically aperiodic or periodic. The Lomb periodogram algorithm in Press et al. (1992) was used to compute the power spectrum of all 17,808 stars in our sample and to determine the "false-alarm probability" (FAP) that the highest peak in the power spectrum could result by chance. The highest frequency searched was $0.5 \mathrm{day}^{-1}$, and the shortest independent frequency was the reciprocal of the time period of the $2000 \mathrm{March}-$ April data sequence for that star. So that at least two full cycles are present in the timeseries data, only periods less than half the total time span of the observations were considered reliable. Stars in the overlap regions of adjacent tiles have more than one photometric measurement recorded per night. Such clumping in the time sampling can invalidate the FAPs, and therefore only one of the overlap measurements was considered. The power spectrum and FAP were computed in this manner for all stars and in each band that contained eight or more reliable photometric measurements.

Because of the multiband nature of our time-series data, the periodicity derived for a star can have high significance under several different scenarios. In generating a list of periodic stars, we nominally required that the false-alarm probability be $\leq 10^{-4}$ in any one of the following combinations: (1) a single band, (2) the product of any two bands, or (3) the product in all three bands. In practice, only one star had an FAP less than $10^{-4}$ in a single band. The effective single-band FAP through this procedure is $\left(10^{-4}\right)^{1 / 2} \approx 0.01$ for two bands and $\left(10^{-4}\right)^{1 / 3} \approx 0.05$ for three bands. When the product of the FAP in two or three bands was used, it was further required that the periods agree to within $20 \%$.

Table 7 summarizes the periods and false-alarm probabilities for the 233 stars identified as periodic variables in this study. Only eight $(3.4 \%)$ of these periodic sources were not identified as variable stars from the Stetson statistic. Conversely, the fraction of the near-infrared variable star population that is periodic is $\sim 18 \%$; however, this is a lower limit to the actual percentage given the conservative FAP limits used to establish periodicity. ${ }^{3}$ If an FAP of $10^{-3}$ is used, an additional $\sim 100$ stars are identified with consistent periods in all three bands. Furthermore, optical moni-

${ }^{3}$ For example, the BN object discussed by Hillenbrand, Carpenter, \& Skrutskie (2001) is not identified as periodic using the adopted FAP criterion.

TABLE 7

Periodic Stars

\begin{tabular}{cccccccc}
\hline \hline & \multicolumn{3}{c}{ PERIOD (days) } & & \multicolumn{3}{c}{ FAP } \\
\cline { 2 - 3 } \cline { 6 - 8 } ID & \multicolumn{1}{c}{$J$} & $H$ & $K_{s}$ & & \multicolumn{1}{c}{$J$} & \multicolumn{1}{c}{$H$} & $K_{s}$ \\
\hline $517 \ldots \ldots$ & 6.52 & 6.16 & 6.25 & & 0.03 & 0.03 & 0.04 \\
$621 \ldots \ldots$. & 2.66 & 2.60 & 2.65 & & 0.07 & 0.05 & 0.03 \\
$1721 \ldots \ldots$ & 5.06 & 4.95 & 5.06 & & $<0.01$ & 0.02 & 0.01 \\
$1936 \ldots \ldots$ & 4.21 & 4.17 & 4.21 & & 0.02 & 0.01 & 0.02 \\
$1994 \ldots \ldots$ & 2.65 & 2.68 & 2.66 & & 0.02 & 0.06 & 0.05 \\
\hline
\end{tabular}

NotE.-Table 7 is presented in its entirety in the electronic edition of the Astronomical Journal. A portion is shown here for guidance regarding its form and content. toring studies have identified $\sim 760$ periodic stars within a $\sim 60^{\prime}$ radius of the Trapezium region of the ONC (Stassun et al. 1999; Herbst et al. 2000b; Rebull 2001), only $\sim 100$ of which are in common with our periodic stars. The $J-H$ versus $H-K_{s}$ color-color diagram for the periodic stars identified in our sample is shown in Figure 21, where it can be seen that most of the periodic stars have colors consistent with WTTSs.

To verify that our criteria produced a reliable sample of periodic stars, robust estimation with replacement (Press et al. 1992) was used to find the number of false periods one would expect to detect among the 17,808 stars in our sample. For each time sample, the observed magnitude was replaced by a magnitude chosen randomly from the timeseries photometry for that star. The $J, H$, and $K_{s}$ photometry were redistributed in parallel, since the coincidence of periodic behavior in these bands was used to select periodic stars from the real data. The power spectra of the redistributed data were computed and the number of significant periodic stars assessed using the same criteria described above. Of the 17,808 stars in the Monte Carlo simulation, only two were identified as periodic.

Finally, we examined the accuracy of our derived periods by comparing them with those found from optical monitoring observations (Stassun et al. 1999; Herbst et al. 2000b; W. Herbst 2000, private communication; Rebull 2001 and references therein). Figure 23 compares the periods for 109 stars identified as periodic at both optical $\left(I_{\mathrm{C}}\right)$ and nearinfrared $\left(J, H\right.$, and/or $\left.K_{s}\right)$ wavelengths. The periods agree to within $10 \%$ for $\sim 80 \%$ of the stars, suggesting that for these stars the origin of the periodicity is the same at optical and near-infrared wavelengths. The biggest discrepancy in the derived periods occurs when our reported period is actually an alias of a more significant period at less than 2 days found from optical monitoring at higher time resolution than our time series. Figure 23 suggests that this occurs for $\sim 10 \%$ of the stars in our periodic sample. Three of the stars with optical periods greater than 10 days have near-infrared periods that differ by a factor of 2 or more. In a few cases, the near-infrared period is approximately half that of the optical period. These may be examples of "period doubling," in which the presence of two starspots on opposite sides of the star causes the periodogram analysis to derive a period half that of the actual value (Herbst et al. 2000b). The other discrepant source (with an optical period of $\sim 60$ days and a near-infrared period of $\sim 14$ days) has been noted to have an uncertain optical period (Rebull 2001).

A histogram of our derived periods is shown in Figure 24. The hatched region indicates periods that are suspected aliases of sub-2 day periods based on comparison with optical monitoring data; only about half of our periodic sample has the information necessary to make this comparison, largely because of our larger spatial coverage compared with optical period searches. The frequency distribution is characterized by a peak at 2-3 days and a slow decline toward longer periods. The range of amplitudes and periods to which our data are sensitive was established by replacing the time-series data for each variable star with a sinusoidal signal plus Gaussian noise that has a dispersion equal to the photometric noise of the actual data. Periodic stars in these simulated data were then identified as described above using a maximum effective FAP of $10^{-4}$. The results indicate that our data are roughly uniformly sensitive to periods between 2 and 10 days, although with 


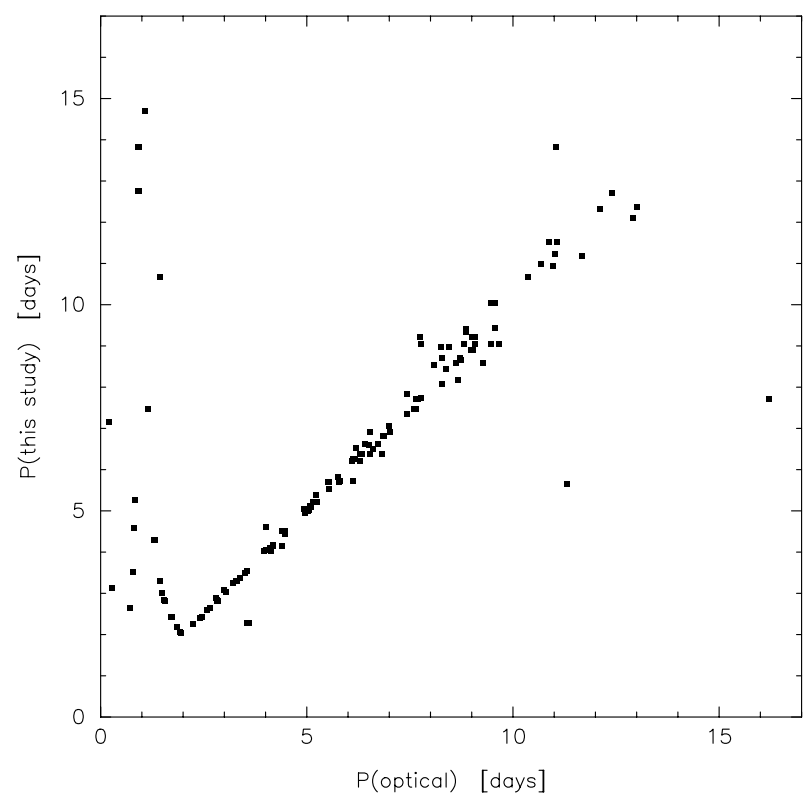

Fig. 23.-Comparison of the optical, I-band periods (Stassun et al. 1999; Herbst et al. 2000b; W. Herbst 2000, private communication; Rebull 2001) with the $H$-band periods derived in this study. For $\sim 80 \%$ of the stars, the optical and near-infrared periods agree to better than $10 \%$. Stars with periods less than $\sim 2$ days as indicated by the optical data are aliased to longer periods in this study because of the 1 day time sampling of the near-infrared observations. Stars that have optical periods roughly twice that of the near-infrared period may be examples of "period doubling," which results when multiple starspots are present (Herbst et al. 2000b). One star with an optical period of $\sim 60$ days and a near-infrared period of $\sim 14$ days is not shown in this figure, since the optical period is uncertain (Rebull 2001).

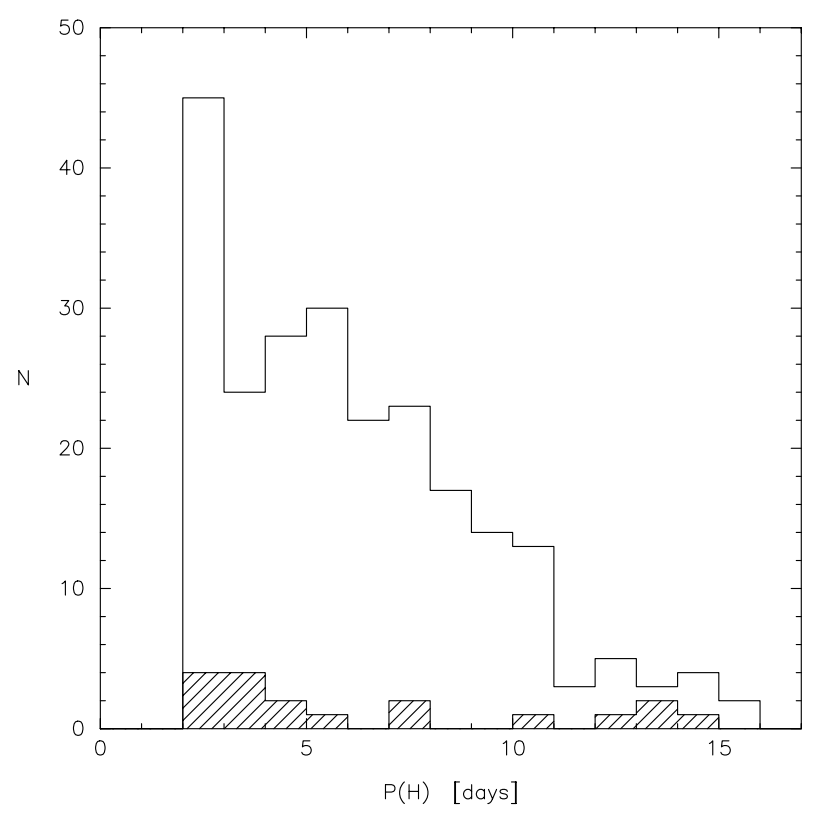

FIG. 24.-Frequency distribution of periods for stars in this study that have false-alarm probabilities less than $10^{-4}$. The open histogram is for all of the stars, and the hatched histogram is stars that are suspected to be aliased with a sub-2 day period based on comparison with the optically derived periods (see Fig. 23). Only about half the stars represented by the open histogram have the necessary information to establish whether the inferred period is aliased in this manner. reduced sensitivity at 2 and 3 days because of the 1 day time sampling of the observations. Approximately half the simulated stars with peak-to-peak amplitudes of $\sim 0.08 \mathrm{mag}$ and $\sim 90 \%$ with peak-to-peak amplitudes of $0.16 \mathrm{mag}$ were identified as periodic in the simulations. By comparison, $55 \%$ of the total variable star population and $60 \%$ of the identified periodic stars have $H$-band peak-to-peak amplitudes $\geq 0.08$ mag.

\section{ORIGINS OF NEAR-INFRARED VARIABILITY}

We turn now from observational characterization of the near-infrared photometric variations to examination of possible physical origins of the variability. The primary observational constraints as established in $\S 5$ are that (1) nearly all of the identified variables are young, low-mass stars associated with the Orion A molecular cloud; (2) the typical timescale for the variability is a few days or less; (3) the light curves exhibit a variety of features, ranging from periodic behavior to discrete variability episodes superposed on otherwise steady light curves, to smooth photometric variations over several days, to month-long (or longer) rises and fades; (4) the amplitudes of the fluctuations are $\sim 0.2 \mathrm{mag}$ on average but can reach $\sim 2 \mathrm{mag}$ in the extreme; and (5) the photometric fluctuations are nearly colorless in most cases, with $\sim 77 \%$ of the variable stars having color variations of less than $0.05 \mathrm{mag}$.

Both the spatial distribution and the observed colors and magnitudes of the variable stars are consistent with what is expected for the Orion pre-main-sequence population, suggesting that much of the observed near-infrared variability is intimately related to the properties of young stellar objects. Further, the short timescale for the photometric fluctuations suggests that the variability originates either within the stellar photosphere or close to it, in the inner circumstellar environment. As established by previous studies (Rydgren \& Vrba 1983; Liseau et al. 1992; Kenyon et al. 1994; Skrutskie et al. 1996; Hodapp 1999), there are several short-term phenomena related to young stellar objects that may contribute to near-infrared variability, including rotational modulation by cool and hot spots on the stellar surface, changes in the line-of-sight obscuration due to circumstellar dust, variations in accretion geometry or mass transfer rates from circumstellar disks, and gradual declines in brightness from EX Lupi or FU Orionis type bursts. Mechanisms not necessarily unique to young stellar objects may also be operative, such as eclipses due to binary companions. Many of these mechanisms were first suspected from optical monitoring observations of young stellar objects (e.g., Herbst et al. 1994; Bouvier et al. 1999). Infrared observations, however, uniquely probe variability related to circumstellar material that radiates at temperatures too cold $(\$ 2000 \mathrm{~K})$ to contribute substantially to optical emission.

In the following sections, we investigate the amplitudes and the timescales expected for photometric fluctuations due to starspots, extinction, and accretion disk phenomena. We begin by analyzing the contributions from cool and hot spots, since these are known to exist on young stars from optical studies and must contribute to near-infrared variability as well. However, as will be shown, starspots cannot explain all of the observed near-infrared variability characteristics, and other mechanisms must be present, with extinction and accretion-related phenomenon as strong candidates. While throughout this discussion we consider 
the effects of these mechanisms separately, in reality a number of mechanisms may be operating simultaneously for any individual star.

\subsection{Starspots}

6.1.1. Models

Starspots, which can be either cooler or hotter than the photospheric temperature, modulate the brightness of a star as stellar rotation alters the fractional spot coverage visible to the observer. Cool spots are thought to arise from magnetic activity on the stellar surface, whereas hot spots are interpreted as regions where material accreting along magnetic field lines (see, e.g., Hartmann, Hewett, \& Calvet 1994) impacts the star. Instead of discrete hot spots, a more realistic model may be one in which the accretion is confined to a high-latitude ring on the stellar surface, where the inclination of the ring with respect to the stellar rotation axis depends on the orientation of the dipole magnetic field (see, e.g., Mahdavi \& Kenyon 1998). In this scenario, variability may result either from simple rotation of the inclined accretion ring around the star or from nonsteady accretion.

The photometric amplitudes expected from both cool and hot starspots were calculated assuming that spots can be characterized by a single-temperature blackbody, $T_{\text {spot }}$, that covers a fraction $f$ of the stellar photosphere with an effective temperature $T_{*}$. The amplitude of the photometric variations, relative to pure photospheric emission, can then be expressed as

$$
\Delta m(\lambda)=-2.5 \log \left\{1-f\left[1.0-B_{\lambda}\left(T_{\text {spot }}\right) / B_{\lambda}\left(T_{*}\right)\right]\right\}
$$

(see, e.g., Vrba et al. 1986), where $B_{\lambda}(T)$ is the Planck function. This starspot model ignores limb darkening, inclination effects, and opacity differences as a function of wavelength between the spot and the stellar photosphere. The models shown in Figure 25 assume a stellar effective temperature of $4000 \mathrm{~K}$, corresponding to a $0.5 M_{\odot}$ star at age 1 Myr (D'Antona \& Mazzitelli 1997), and cool- and hot-spot temperatures of 2000 and $8000 \mathrm{~K}$, respectively. Results are presented for spots that cover $1 \%, 2 \%, 5 \%$, $10 \%, 20 \%$, and $30 \%$ of the stellar surface. These models encompass the more extreme spot parameters inferred from optically selected samples of low-mass pre-main-sequence stars (Bouvier \& Bertout 1989; Bouvier et al. 1993; Fernández \& Eiroa 1996).

Figure 25 shows that cool and hot spots can be distinguished observationally in the near-infrared based on the amplitude of the photometric fluctuations. While both hot and cool spots with small fractional coverage produce lowamplitude, nearly colorless fluctuations, the maximum amplitude from cool spots is $\sim 0.4 \mathrm{mag}$ at $J$ band, while hot spots can produce photometric fluctuations as large as 1 mag for sufficiently hot spot temperatures, large fractional coverages, or both. Further, the maximum change in the $J-H$ and $H-K_{s}$ colors that can be produced with the spot parameters considered here is $\sim 0.03$ mag for cool spots but 0.1-0.2 mag for hot spots.

The timescale for variability caused by cool or hot starspot modulation is governed by the stellar rotation period. ${ }^{4}$

\footnotetext{
${ }^{4}$ Smith, Bonnell, \& Lewis (1995) suggested, though, that the period measured from hot spots may represent the beat frequency between the stellar rotation frequency and the orbital frequency at the magnetospheric boundary.
}

The frequency distribution of periods in $\mathrm{T}$ Tauri stars derived from optical monitoring observations implies rotation periods of $\lesssim 10$ days for $\sim 90 \%$ of the known periodic stars in Orion (Stassun et al. 1999; Herbst et al. 2000b; W. Herbst 2000, private communication; Rebull 2001), assuming that the observed periodicity is a result of rotational modulation by starspots. Similar rotation periods have been derived from Doppler imaging of $\mathrm{T}$ Tauri stars (Joncour, Bertout, \& Bouvier 1994; Rice \& Strassmeier 1996; Johns-Krull \& Hatzes 1997; Neuhäuser et al. 1998). Rotational velocities derived from high-resolution spectroscopy $(v \sin i)$ also imply that young stellar objects have rotational periods on the order of a few days (Bouvier et al. 1986; Hartmann et al. 1986). Timescales of a few days are consistent with the results of our ACF analysis (\$ 5.3.1).

Cool and hot spots are perhaps distinguishable based not on the timescale of the photometric variability, but on the timescale over which it persists. Cool spots are thought to be relatively stable features that can last several years or more. Hence, observed periodicity is often a repeatable result, especially in WTTSs, which do not have additional variability components related to accretion phenomena. Hot spots, however, generally last only a few days or weeks, as evidenced by period changes and even disappearance and reappearance in a few cases where hot-spot periods have been detected (Vrba et al. 1989, 1993). Thus, hot spots tend to produce irregular variability, especially if the accretion of material onto the star is unsteady, as has been inferred in some stars (Gullbring et al. 1996; Basri, JohnsKrull, \& Mathieu 1997; Smith et al. 1999), or if the geometry is complicated by misaligned rotation and magnetic dipole axes (see, e.g., Mahdavi \& Kenyon 1998; Bouvier et al. 1999). Based on these tendencies, Herbst et al. (1994) introduced a classification scheme in which periodic fluctuations from cool spots (mainly in WTTSs, but in some CTTSs as well) are type I variables, irregular fluctuations in CTTSs from hot spots are type II variables, and periodic fluctuations in CTTSs from hot spots are type IIp variables (type III variability in this classification scheme is discussed in $\S 6.3$ ).

\subsubsection{Comparison with Observations: Cool Spots}

For cool-spot parameters typically inferred from optical observations $\left(T_{*}-T_{\text {spot }} \lesssim 2000 \mathrm{~K}\right.$ and $f \lesssim 30 \%$; see Bouvier \& Bertout 1989; Bouvier et al. 1993; Fernández \& Eiroa 1996), the expected peak-to-peak amplitudes should be $\lesssim 0.4 \mathrm{mag}$ in the $J, H$, and $K_{s}$ bands and $\lesssim 0.03 \mathrm{mag}$ in the $J-H$ and $H-K_{s}$ colors (Fig. 25). These amplitudes are only approximate, given the simplicity of the model, with the essential predictions that the color variations from cool spots should be relatively low and appear periodic (more so in WTTSs than CTTSs) by analogy with type I optical variability (Herbst et al. 1994; see also Herbst, Maley, \& Williams 2000a). In our data, $65 \%$ of the periodic variable stars have amplitudes $\leq 0.4 \mathrm{mag}$ at $J$ band and $\leq 0.03 \mathrm{mag}$ in the $J-H$ color, $77 \%$ have color amplitudes $\leq 0.05 \mathrm{mag}$, and $80 \%$ have near-infrared colors consistent with WTTSs. Thus, rotational modulation by cool spots can account for the variability characteristics in the majority of the periodic stars.

Periodic stars, however, account for only $\sim 18 \%$ of the total variable population, and an additional $\sim 600$ stars ( $\sim 50 \%$ of the identified variables) also have low-amplitude, nearly colorless photometric fluctuations but have not been 

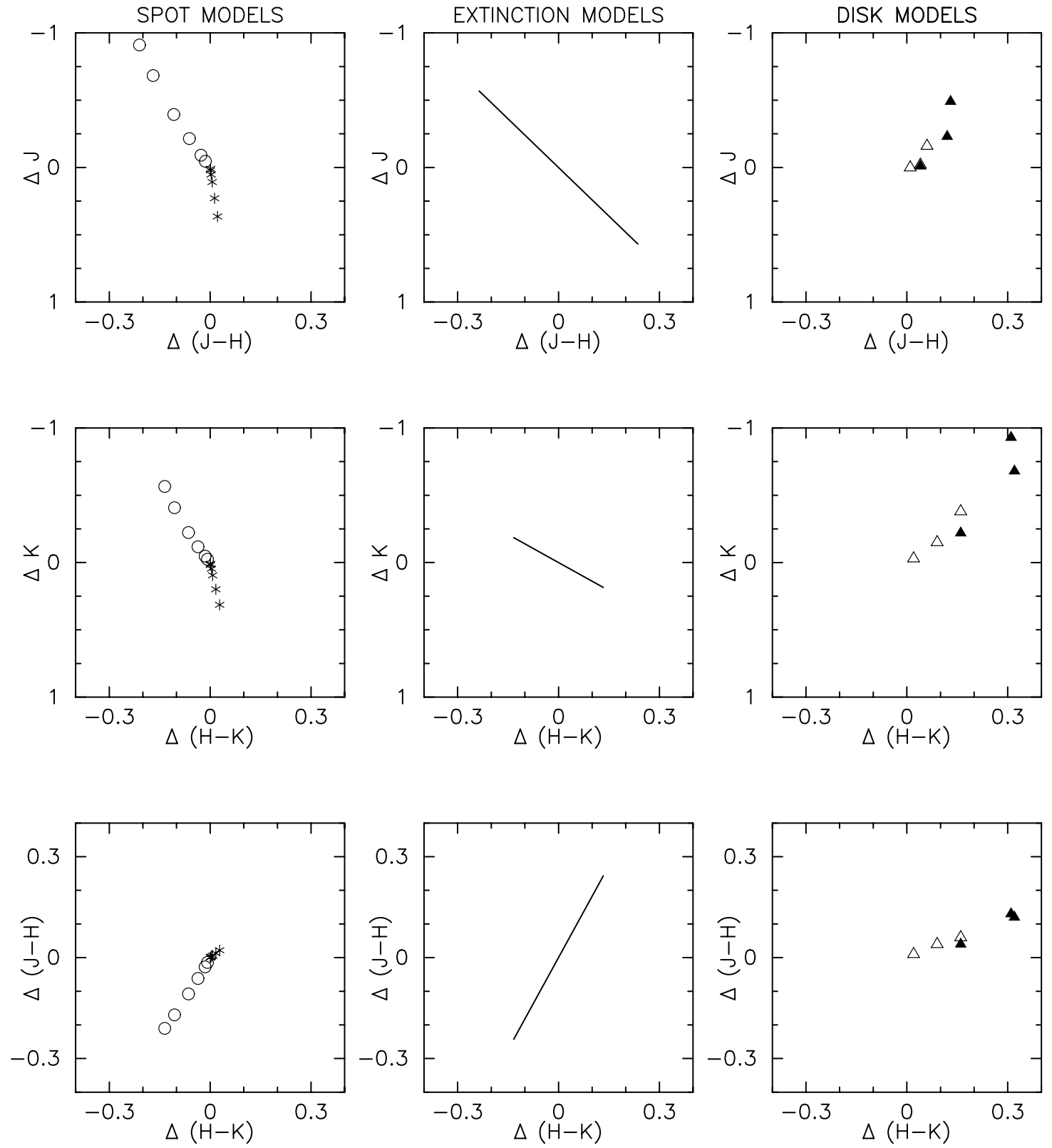

Fig. 25.-Model $J$ vs. $J-H, K_{s}$ vs. $H-K_{s}$, and $J-H$ vs. $H-K_{s}$ diagrams for cool and hot spots, extinction, and accretion disk variations. These models can be compared with the observed diagrams in Figs. 4-13 to investigate the origin of the near-infrared variability. The models assume a photospheric temperature of $4000 \mathrm{~K}$, appropriate for a $1 \mathrm{Myr}, \sim 0.5 M_{\odot}$ star (D’Antona \& Mazzitelli 1997), spot temperatures of $2000 \mathrm{~K}$ for cool spots (asterisks) and 8000 $\mathrm{K}$ for hot spots (circles), and spot coverages of $1 \%, 2 \%, 5 \%, 10 \%, 20 \%$, and $30 \%$ (see eq. [7]). The extinction vectors were calculated using the interstellar reddening law from Cohen et al. (1981), transformed into the 2MASS color system (Carpenter 2001). The lengths of the vectors correspond to $\Delta A_{V}= \pm 2$ mag. The disk models have been provided courtesy of $\mathrm{N}$. Calvet and represent the effects of varying the mass accretion rate and inner hole size of the accretion disks. Results are presented for mass accretion rates of $10^{-8.5} M_{\odot} \mathrm{yr}^{-1}$ (open triangles) and $10^{-7.0} M_{\odot} \mathrm{yr}^{-1}$ (filled triangles), and for each accretion rate, inner hole sizes of 1,2 , and $4 R_{\odot}$. The larger hole sizes correspond to smaller infrared excesses.

identified as periodic. While arbitrarily low photometric fluctuations can be produced by many of the physical mechanisms discussed in this section, Figure 26 suggests that rotational modulation by cool spots may cause much of the low-amplitude variability, independent of whether a period is actually detected. This figure shows the $J-H$ versus $H-K_{s}$ color-color diagram as a function of the peak-to-peak $J$-band amplitude. Most stars with low amplitudes $(\leq 0.2 \mathrm{mag}$ ) have near-infrared colors consistent with WTTSs or stars with small infrared excesses. As the $J$-band amplitude increases, the near-infrared colors tend to become redder and an increasing fraction contain a nearinfrared excess characteristic of the optically thick circumstellar disks of CTTSs. This trend suggests that different mechanisms related to the absence or presence of an accretion disk may be producing the low- and high-amplitude variability. Of the variability mechanisms examined here, only cool spots and eclipsing binaries do not require the presence of a circumstellar disk. Since eclipsing systems cannot account for the large number of stars with lowamplitude variability, cool spots appear to be a more likely explanation. 


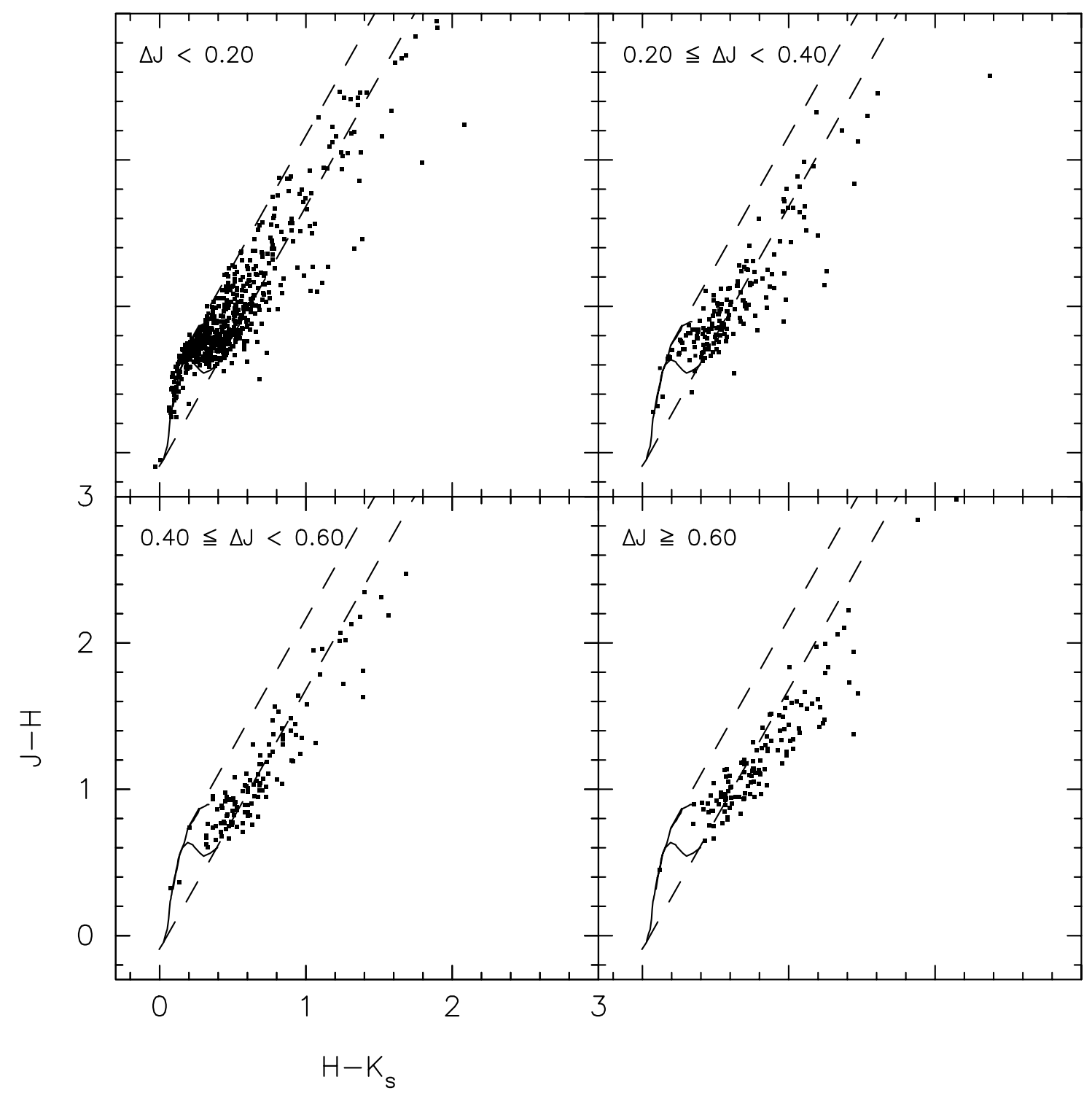

FIG. 26.-The $J-H$ vs. $H-K_{s}$ diagrams for variable stars as a function of the $J$-band peak-to-peak amplitude. This figure shows that stars with larger $J$-band amplitudes tend to have redder colors and larger near-infrared excesses than stars with smaller fluctuations.

Cool-spot modulation produces larger amplitude fluctuations at optical wavelengths, and so optical surveys should provide a more complete census of the periodic variables. As mentioned in $\S 5.3 .2$, optical studies have found $\sim 760$ periodic stars in the Orion Nebula region (Stassun et al. 1999; Herbst et al. 2000b; W. Herbst 2000, private communication; Rebull 2001), of which $\sim 330$ are identified as near-infrared variables and 109 as periodic in this study. Of those optical periodic stars also identified as near-infrared variables (but not necessarily periodic near-infrared variables), $\sim 70 \%$ have low peak-to-peak amplitudes in the $J$-band magnitudes $(\leq 0.4 \mathrm{mag})$ and $J-H$ colors $(\leq 0.03$ mag). Further, $\sim 88 \%$ of the $\sim 650$ optical periodic variables not identified as periodic in the near-infrared have near-infrared colors consistent with reddened WTTSs.

Based on the observational evidence just described, we speculate that the photometric fluctuations observed in many of the low-amplitude variable stars are due to coolspot modulation. As a lower limit to the stars that may have variability due to cool-spot modulation, we find that $57 \%$ of the variables have $J$-band amplitudes $\leq 0.4 \mathrm{mag}, J-H$ amplitudes $\leq 0.03 \mathrm{mag}$, and no near-infrared excess, as expected for cool-spot parameters inferred from optical observations. Given the simplicity of the starspot model, if we crudely assume that the maximum $J-H$ color change that can be produced by cool spots is $0.05 \mathrm{mag}$, we derive an upper limit of $77 \%$ as the percentage of the variability that may be attributed to cool spots.

\subsubsection{Comparison with Observations: Hot Spots}

While cool spots can plausibly account for the lowamplitude variables with small color variations, they cannot explain the $23 \%$ of the variable stars that have color variation exceeding $\sim 0.05 \mathrm{mag}$ (see Fig. 25 ). Stars with significant color variations were analyzed in $\S 5.2$, where it was shown that these objects tend to have colors consistent with CTTSs (see Fig. 21). Therefore, again by analogy with optical variability characteristics, we investigate whether hot spots, which cause type II optical variability in CTTSs in the Herbst et al. (1994) classification scheme, can account 
for the color amplitudes observed in some of the nearinfrared variable stars.

For hot-spot parameters typically inferred from optical observations $\left(T_{\text {spot }} \lesssim 8000 \mathrm{~K}, f \lesssim 10 \%\right)$, hot spots can cause peak-to-peak amplitudes at near-infrared wavelengths as large as $\sim 0.2-0.4 \mathrm{mag}$ in the magnitudes and $\lesssim 0.06-0.12$ mag in the colors (see Fig. 25). About $10 \%$ of the nearinfrared variable stars have magnitude and color amplitudes larger than these hot-spot predictions (see $\S 5.2$ ). A combination of hotter spot temperatures $(\gtrsim 10,000 \mathrm{~K})$ and higher coverages $(\gtrsim 20 \%)$ is needed to explain these stars with hot spots alone. These spot parameters are evidently rare, but they may be possible and simply not have been identified previously because of small number statistics that are overcome by the extensive near-infrared variable sample obtained here.

Because of the substantial color variability expected from hot spots, a more quantitative comparison between the observations and the hot-spot model can be made by assessing correlated color and magnitude changes in individual stars. Figure 20 showed the derived slopes in various color-color and color-magnitude diagrams, as discussed in $\S 5.2$. The predicted slope from the hot-spot model is also indicated in this figure, which varies by only a few kelvins for stellar effective temperatures between 3000 and $6000 \mathrm{~K}$ and spot temperatures up to $40,000 \mathrm{~K}$. Figure 20 shows that many of the observed slopes in the $J$ versus $J-H$ diagram can be accounted for quantitatively by hot spots (and extinction variations, as discussed in the following section). However, in the $J-H$ versus $H-K_{s}$ and $K_{s}$ versus $H-K_{s}$ diagrams, while there is an approximate correspondence between the observed and predicted slopes, the observed slopes are systematically shallower than would be expected if the variability were due solely to hot spots. These differences may not be significant, though, given the simplicity of the hot-spot model. In addition, the $K_{s}$ versus $H-K_{s}$ diagram contains a number of stars with negative slopes, in that the colors become bluer as the star gets fainter. Such variations are completely inconsistent with the hot-spot model and suggest that an additional mechanism or mechanisms are present that contribute to the nearinfrared variability, especially at $K_{s}$ band.

\subsection{Extinction}

\subsubsection{Models}

Variability due to extinction can result from inhomogeneities in either the inner circumstellar environment or the ambient molecular cloud that move across the line of sight. Photometric changes induced by visual extinction variations of $\Delta A_{V}= \pm 2 \mathrm{mag}$ are illustrated in the middle column in Figure 25, although the extinction changes in practice can be of arbitrary value. The extinction vectors were calculated from the interstellar reddening law measured by Cohen et al. (1981) and transformed into the 2MASS photometric system (Carpenter 2001). If the extinction variations originate from a circumstellar disk and the dust grains have conglomerated into larger particles to produce a more gray extinction law, the amplitude of the color variations will be less than indicated. For the interstellar reddening law, Figure 25 shows that extinction variations produce photometric slopes at near-infrared wavelengths similar to those expected from hot spots, except in the $K_{s}$ versus $H-K_{s}$ diagram, where the extinction slope is shallower by $\sim 25^{\circ}$.
The timescale for extinction variations caused by the ambient molecular cloud is set by the size scale of the structural inhomogeneities and the velocity field of the molecular gas. The typical line-of-sight full width at maximum line width observed in the Orion molecular cloud from singledish ${ }^{13} \mathrm{CO}(1-0)$ observations is $\sim 2 \mathrm{~km} \mathrm{~s}^{-1}$ (Bally et al. 1987). Thus, the timescale for one point in the cloud to transit a stellar diameter of $3 R_{\odot}$ (representative of a $\sim 0.5$ $M_{\odot}$ star at an age of $1 \mathrm{Myr}$ ) is $\sim 12$ days. The line wings in molecular line profiles can extend to several kilometers per second, which may shorten the appropriate timescales to a few days.

If structural inhomogeneities in a circumstellar disk occult the star to cause extinction variations, a variety of timescales are possible. Possible inhomogeneities include azimuthal asymmetries in the plane of the disk, a warped or flared outer disk (Bertout 2000), and a warped or precessing inner disk (Lai 1999; Bouvier et al. 1999; Terquem \& Papaloizou 2000). Azimuthal asymmetries or warped features will produce variability on timescales dictated by the rotational velocity of the disk and the size scales of the inhomogeneous features. The time for one position in the disk to transit the star ranges from hours within a few solar radii to a week or more for disk radii $\gtrsim 30$ AU. (If a warp extends for a significant azimuthal distance around the star, however, the relevant timescale will be given by the rotation period at that radius.) The fraction of the stars that show extinction variability will depend on the probability that these inhomogeneous features transit the star. These predictions are heavily model dependent, but Bertout (2000) suggests that as many as $20 \%$ of the stars with circumstellar disks may be occulted by the flared outer disk. Azimuthal asymmetries require viewing the disk nearly edge-on and are not likely to contribute substantially to the total number of variables. The magnetospheric accretion columns and the inner disk wind are likely optically thin and would not contribute to extinction variations (Kenyon, Yi, \& Hartmann 1996 and references therein).

\subsubsection{Comparison with Observations}

If the ambient molecular cloud produces extinction variations, then the variable star population exhibiting significant color variations should contain a mixture of all (nonforeground) populations to which our survey is sensitive. This would include background field stars and Orion population stars with and without near-infrared excesses (i.e., CTTSs and WTTSs). Since field stars and WTTSs outnumber CTTSs, CTTSs should be the least represented group in the variable star population. However, as shown in the left panel of Figure 21, stars with large color variations preferentially have near-infrared excesses characteristic of circumstellar disks and CTTSs. This, combined with the longer timescale ( $\sim 12$ days) expected for cloud transit events compared with the typical observed variability timescale ( $\lesssim 3$ days; see Fig. 22) suggests that if extinction changes do contribute to near-infrared variability, the source of the extinction is in the inner circumstellar environment and not the ambient cloud.

Visual extinction variations of $\sim 10 \mathrm{mag}$ are needed to account for the extreme magnitude and color variations indicated in Table 6 . The typical observed variability timescale of $\lesssim 3$ days indicates that any inhomogeneities in the disk structure must transit the star within a similar interval. For a stellar radius of $1.5 R_{\odot}$, the implied velocity of the 
inhomogeneous region is $\gtrsim 10 \mathrm{~km} \mathrm{~s}^{-1}$. Assuming Keplerian rotation, the maximum radial distance from a $0.5 M_{\odot}$ star that produces such velocities is $\sim 7 \mathrm{AU}$. If the size of the warp is significantly larger than the stellar diameter, the warp would need to be located at smaller radial distances to be consistent with the observed variability size scale. This suggests that the outer disk flares described by Bertout (2000) are not likely responsible for the short-term photometric variability observed here, but they may still contribute to the longer term fluctuations.

The slope of the photometric correlations shown in Figure 20 can also be used to test whether the observed variability may be due to extinction variations. The expected slope of the interstellar extinction law is indicated by the filled squares in Figure 20. This figure shows that extinction variations (along with hot spots) can quantitatively explain most of the observed slopes in the $J$ versus $J-H$ diagram. However, as with the hot-spot model, extinction cannot in detail account for the observed slopes in the $J-H$ versus $H-K_{s}$ and $K_{s}$ versus $H-K_{s}$ diagrams. In the color-color diagram, the observed slopes are not as steep as expected from extinction. In the $K_{s}$ versus $H-K_{s}$ diagram, while extinction can account for many of the observed slopes, the average observed slope is steeper than expected for interstellar extinction and shallower than predicted by hot spots. Invoking a more gray extinction (although not completely gray, as color variability is observed) would provide better agreement with the observed slopes in the $J-H$ versus $H-K_{s}$ and $K_{s}$ versus $H-K_{s}$ diagrams, but then we could not simultaneously account for the already good agreement with the $J$ versus $J-H$ slopes. Therefore, extinction cannot simply account for the observed variability characteristics. Further, similar to hot spots, extinction cannot explain any of the stars that have negative slopes in the $K_{s}$ versus $H-K_{s}$ diagram.

\subsection{Accretion Disks}

The above discussion indicates that the simple starspot and extinction models cannot explain all of the variability characteristics of stars that exhibit color variations. The two main discrepancies are the slopes of the positive photometric correlations in the $J-H$ versus $H-K_{s}$ and $K_{s}$ versus $H-K_{s}$ diagrams (but not the $J$ vs. $J-H$ diagram), and any of the stars with negative slopes in the $K_{s}$ versus $H-K_{s}$ diagram. This result suggests that an additional mechanism may be present that affects the emission at longer wavelengths more so than at shorter wavelengths. Since the stars that cannot be explained by the starspot and extinction models tend to have near-infrared excesses, we consider emission from a circumstellar disk as a source of nearinfrared variability.

\subsubsection{Models}

Disks contribute to the near-infrared emission through two means. First, they absorb radiation from the star and reradiate it at longer wavelengths, producing the infrared excess that is commonly observed in young stellar objects. The shortest wavelength at which a near-infrared excess is produced depends on how close the disk extends to the stellar surface and how hot the inner disk material becomes (Meyer et al. 1997). A second source of near-infrared emission from circumstellar disks occurs when energy is released as material is radially transported through a viscous, optically thick accretion disk (Lynden-Bell \& Pringle 1974).
Photometric variability due to a circumstellar disk can then result from changes in the mass accretion rate and, consequently, the accretion luminosity ${ }^{5}$ or changes in the inner disk structure that alter the amount of absorbed and reprocessed stellar radiation. Changes in the reprocessed radiation may result, for example, from physical variations in the disk inner hole radius, or variations in the thickness of the inner edge of a warped disk. Such complicated inner disk geometries are predicted to occur when the dipole magnetic field is misaligned with the stellar rotation axis (Mahdavi \& Kenyon 1998; Lai 1999; Terquem \& Papaloizou 2000).

Figure 25 shows the expected photometric variations for changes in the mass accretion rate and inner hole radius of a circumstellar disk around a star with an effective temperature of $4000 \mathrm{~K}$. These model results are from the same models used by Meyer et al. (1997) and were kindly provided by N. Calvet. The open and filled triangles in Figure 25 represent mass accretion rates of $\dot{M}=10^{-8.5}$ and $10^{-7.0}$ $M_{\odot} \mathrm{yr}^{-1}$, respectively, and for each accretion rate, the triangles represent the photometric emission for an inner hole radius of 1,2 , and $4 R_{\odot}$, all for a disk inclination angle of $45^{\circ}$. As the inner hole radius increases, the star-disk system becomes fainter in the near-infrared, with less emission absorbed and reprocessed by the circumstellar disk. However, unlike the hot-spot and extinction models, the system colors become bluer (approaching the stellar color) as it becomes fainter, since the disk radiates more strongly at $K_{s}$ band than at $J$ or $H$. For hole sizes $\gtrsim 4 R_{\odot}$ and a mass accretion rate of $\sim 10^{-8.5} M_{\odot} \mathrm{yr}^{-1}$, no near-infrared excess emission is produced. The photometric variations expected from these disk models can be as large as 1 mag at $K$ band for the hole sizes and accretion rates illustrated in Figure 25. Photometric variations of several magnitudes can be achieved if the accretion rates increase to $\dot{M} \sim 10^{-5}$ to $10^{-4} M_{\odot} \mathrm{yr}^{-1}$. These accretion disk models also predict a shallower slope in the $J-H$ versus $H-K_{s}$ diagram than expected from extinction or hot-spot variability, which is consistent with the observed locus of CTTSs in this colorcolor diagram (Meyer et al. 1997).

To examine the timescales associated with viscous accretion disks, we considered an accretion disk around a 0.5 $M_{\odot}$ star using the $\alpha$ viscosity parameterization (Shakura \& Sunyaev 1973) and assuming $\alpha=0.01$ (Hartmann et al. 1998). The accretion rate can change globally within the disk or in a localized region. The timescale to transport material from the outer to the inner disk is $\gtrsim 1000 \mathrm{yr}$ for $\dot{M} \sim 10^{-8} M_{\odot} \mathrm{yr}^{-1}$ and radii greater than $1 \mathrm{AU}$ (Frank, King, \& Raine 1985). Accretion rates may also vary if the disk equilibrium is perturbed. Thermal instabilities in the disk can be on the order of $\sim 1$ day in the inner disk region given the uncertainty in $\alpha$ (Frank et al. 1985). Another possible mechanism that may lead to variable mass accretion rates in the inner disk is a time-variable magnetic field. In the current paradigm of magnetospheric accretion, the size of the inner hole of a circumstellar disk in steady state is a function of the mass accretion rate and the stellar magnetic

\footnotetext{
${ }^{5}$ Herbst \& Shevchenko (1999) suggested that changes in the accretion luminosity produce the irregular optical photometric variability sometimes observed in stars more massive than typical CTTSs, or type III variability in the Herbst et al. (1994) classification scheme. Grinin, Rostopchina, \& Shakhovskoi (1998), however, attribute type III variability to variable circumstellar extinction.
} 
field strength (Königl 1991). If the magnetic field strength varies in time, the magnetosphere will intersect the disk at a radius other than the corotation radius, which will modulate the accretion flow in the inner disk. Armitage (1995) and Clarke et al. (1995; see also Kenyon et al. 1996) invoked this model to explain the variability characteristics in $\mathrm{T}$ Tauri stars over many years, but since the viscous timescale in the inner disk approaches $\sim 10$ days for large values of $\alpha$, this may contribute to shorter term variability as well. Finally, the growth time of a warping instability in the inner disk, which may lead to variations in the amount of reprocessed emission, is $\sim 6$ days (Lai 1999).

\subsubsection{Comparison with Observations}

The observed timescales of the near-infrared fluctuations rule out any global changes in the disk mass accretion rate (expected on timescales $\gtrsim 10^{3} \mathrm{yr}$, if indeed occurring) as the origin of the near-infrared variability observed here. Shortterm variations in the mass accretion rate or geometry in the inner disk are somewhat speculative, and it is not clear that they can in fact produce variability on timescales of days. Nonetheless, the following discussion considers shortterm variations in the disk properties as possible origins of the observed near-infrared photometric fluctuations.

The accretion disk models plotted in Figure 25 reproduce the observed locus of CTTSs in the $J-H$ versus $H-K_{s}$ color-color diagram (Meyer et al. 1997), and this observed CTTS locus slope is indicated in Figure 20. In the $J-H$ versus $H-K_{s}$ diagram, the variable stars follow an observed slope that is steeper on average than expected from a time-variable disk (although extinction and hot spots are no more successful in uniquely explaining the observed slopes). In the color-magnitude diagrams, the observed slopes are positive, while the predicted slopes from the disk models are distinctly negative. Thus, the majority of the stars exhibiting color variability cannot have their variability explained solely by these disk models. However, a small number of stars $(\sim 17)$ do have negative observed slopes in the $K_{s}$ versus $H-K_{s}$ diagram (see, e.g., Fig. 8) that agree quantitatively with expectations from the disk models. In fact, seven of the 17 stars with significant negative slopes may have a near-infrared excess indicative of a circumstellar disk. About half of the stars with negative slopes do not show a near-infrared excess, although it should be noted that not all stars with $K_{s}$ excesses will be identified in the $J-H$ versus $H-K_{s}$ diagram.

Finally, we briefly discuss the limits set by our data on the frequency of FU Orionis type bursts, in which a star brightens by several magnitudes in $\lesssim 1$ year, presumably as a result of a dramatic increase in accretion driven by largescale disk instabilities (Hartmann, Kenyon, \& Hartigan 1993). All stars brighter than $K_{s}=14$ in the 2000 MarchApril data are apparent in the 1998 March images, and the largest observed change in the $K_{s}$-band magnitudes is 1.7 mag, with an approximately equal number of stars decreasing in brightness by over 1 mag as increasing over this $\sim 2$ year period. Thus, no convincing evidence exists for an FU Orionis type burst in these monitoring observations. A lower limit on the timescale for such bursts is given then by the number of stars in the Orion A molecular cloud ( 2700; see Appendix) and the time spanned by the observations ( $\sim 2$ yr), or $\gtrsim 5400$ years. This is consistent with the timescale between FU Orionis bursts commonly assumed ( $\sim 10^{4}$ yr; Herbig 1977; Hartmann et al. 1993).

\subsection{Eclipsing Systems}

Although the above mechanisms are the leading candidates to account for most of the observed near-infrared variability, a small number of systems have variability properties consistent with eclipsing binary systems. This type of variability is distinguished by simultaneous drops in the $J, H$, and $K_{s}$ amplitudes on a single observation for the 1 day time interval of our observations (e.g., BM Ori in Fig. 6). As an initial screening for potential eclipsing systems, the 2000 March-April data were searched for stars in which at least two of the three bands decreased in brightness by more than $5 \sigma$ from the mean magnitude, where $\sigma$ is the observed photometric rms after excluding the faintest $20 \%$ of the measurements (so as to exclude the actual eclipses from the rms measurements). These criteria yielded 73 stars, all of which were previously identified as variable based on the Stetson index or other criteria. The light curves for these stars were then visually inspected to identify possible eclipsing systems. The 22 candidate eclipsing systems in our sample are listed in Table 4. All but two of these stars are located within the spatial boundaries of the stellar density enhancement identified here (see Appendix) and are likely pre-main-sequence stars. If these stars can be verified as eclipsing systems and followed up spectroscopically to determine the orbital elements, they can provide a valuable means to measure the stellar masses and test pre-mainsequence evolutionary tracks. Only one candidate in our list, BM Ori, is known to be an eclipsing system from previous studies (e.g., Antokhina, Ismailov, \& Cherepashchuk 1989). For the remaining sources, either the drop in the $J$, $H$, and $K_{s}$ amplitudes does not repeat within the time range covered by these observations, or the star exhibits additional variability characteristics such that the amplitude drops may be attributed to other phenomena. The eclipsing nature of these systems thus remains to be confirmed.

\section{DISCUSSION}

Although the current data are not sufficient to uniquely identify the predominant variability mechanisms on a starby-star basis, we can make a few general statements about the origins of the observed near-infrared variability. Rotational modulation by cool spots is the leading candidate for the low-amplitude variables, since many of these stars have photometric characteristics analogous to type I optical variability (Herbst et al. 1994). Approximately $56 \%-77 \%$ of the variable stars may have variability attributed to coolspot modulation. The origin of the variability in stars that exhibit color changes is not clear. Hot spots, extinction, and accretion disk phenomenon can explain the coarse properties of these stars, but the detailed correlation between the colors and magnitudes cannot be accounted for by any single mechanism. The largest discrepancy is among photometric correlations involving the $K_{s}$-band photometry $\left(J-H\right.$ vs. $H-K_{s}$ and $K_{s}$ vs. $\left.H-K_{s}\right)$, indicating that relatively cool variability phenomena are present in many of the stars. This suggests that either (1) the hot-spot and extinction models used here are too simplistic, (2) a process not identified in this study is contributing to the variability, or (3) several of these mechanisms are operative in any individual star. It would not be surprising, and could possibly even be expected, that more than one variability mechanism is present, since several of the identified phenomena (hot spots, extinction, and accretion variability) are all 
related to the presence of a circumstellar disk. Because extinction tends to make stars fainter, while hot spots and accretion tend to make stars brighter, the net result upon the photometric properties of any individual star is not easy to predict.

Evidence for multiple variability mechanisms within a single star is in fact available for a few objects. YY Ori, shown in Figure 8, is the namesake for a class of objects displaying broad, inverse P Cygni absorption lines that indicate matter infalling onto the star at nearly free-fall velocities (Walker 1972). YY Orionis stars generally have ultraviolet excesses (Walker 1983), presumably a result of the accretion shock that develops when infalling material impacts the star. YY Ori is suspected to contain a hot spot on the basis of optical observations (Bertout et al. 1996), which will also contribute to near-infrared variability. However, the near-infrared observations are best explained by variations in the accretion disk properties (see Fig. 8). ${ }^{6}$ Other stars in our survey show evidence for multiple variability mechanisms within the actual near-infrared data. The star shown in Figure 10, for example, initially shows photometric fluctuations most consistent with extinction but then shows a clear near-infrared excess that subsequently disappears. As another example, the long-term variable shown in Figure 13 faded at $J$ band over a 2 year time period while simultaneously brightening at $K_{s}$ band. This behavior cannot be produced by any single variability mechanism considered here.

Finally, we briefly discuss the implications that nearinfrared variability has for determining the stellar mass and age distributions in young star-forming regions. These distributions are frequently established either by modeling the observed colors and magnitudes of the young stars (e.g., Comerón, Rieke, \& Neuhäuser 1999; Muench, Lada, \& Lada 2000; Hillenbrand \& Carpenter 2000) or by combining photometric and spectroscopic observations (e.g., Greene \& Meyer 1995; Meyer 1996; Luhman et al. 1998). Variability can influence the results, since it sets a limit on how accurately the luminosity of the star can be measured. Assuming that the near-infrared variability characteristics established here are typical of most star-forming regions, our results suggest that variability will have a relatively minor effect in analyzing the mass and age distributions for the majority of the stellar population. For a $1 \mathrm{Myr}, 0.5 M_{\odot}$ star, the average $J$-band dispersion of $0.09 \mathrm{mag}$ translates into a mass and an age uncertainty of $\sim 10 \%$ (D'Antona \& Mazzitelli 1997), which are minor relative to current uncertainties in pre-main-sequence evolutionary tracks. Given that most stars in the Orion A molecular cloud are not detected as variable in this study, the mass and age uncertainties for the typical star will be even less. Even for stars that exhibit large amplitude variability, the color variations roughly follow the interstellar reddening law, suggesting that simultaneous observations can reasonably estimate the extinction from the observed colors and spectral type (assuming the spectral variability is negligible). However, if the broadband photometric observations are taken on

\footnotetext{
${ }^{6}$ The light curves of other YY Orionis stars within our survey region (Walker 1972; Harder, Bertout, \& Mennessier 1998) were examined to see if they also showed negative slopes in the $K_{\mathrm{s}}$ vs. $H-K_{s}$ diagram. One (NS Ori) shows photometric trends similar to, but more complex than, those of YY Ori, and three others (CE Ori, SU Ori, and XX Ori), while detected as variable stars in our data, do not have negative slopes.
}

separate days, or if the stars exhibit spectral as well as photometric variability, photometric errors of up to $\sim 2$ mag may be introduced in estimating the stellar luminosities, which can lead to mass errors of a factor of 2-3 and age errors of up to an order of magnitude for a $1 \mathrm{Myr}, 0.5$ $M_{\odot}$ star. Only a small percentage of the stars should be affected in this manner, indicating that one must consider variability in interpreting the tails of mass and age distributions (see also Hartmann 2001).

\section{SUMMARY}

We have investigated the $J, H$, and $K_{s}$ near-infrared variability characteristics of stars in a $\sim 0.84 \times 6^{\circ}$ region centered on the Trapezium region of the Orion Nebula cluster using the southern 2MASS telescope. The primary set of observations analyzed here were conducted on nearly a nightly basis for a 1 month time period in 2000 MarchApril, but our observations also include data from 1998 March and 2000 February. These data are used to establish the near-infrared variability characteristics of young stars on timescales of $\sim 1-36$ days, $\sim 2$ months, and $\sim 2$ years, and to investigate possible mechanisms that may cause near-infrared variability in pre-main-sequence objects.

Variable stars were identified primarily by quantifying the correlated photometric fluctuations in the $J, H$, and $K_{s}$ bands using the Stetson (1996) variability index. The light curves of the identified stars exhibit a diversity of features including periodic fluctuations, continuous aperiodic variations over day-long timescales, long-term variability over $\sim 2$ years, steady rises or fades, photometric fluctuations on discrete days imposed on otherwise constant brightness (including eclipses), colorless variability, stars that become redder as they fade, and stars that become bluer as they fade. Examples of many of these phenomenon can be found in Figures 4-13, and light curves for all identified variable stars can be found in the electronic version of this article.

In total, 1235 variable stars were identified, compared with the estimated $\sim 2700$ stars with magnitudes $K_{s} \leq 14$ that are associated with the Orion A molecular cloud. The observed spatial distribution, magnitude, and colors suggest that $\sim 93 \%$ of the variables are pre-main-sequence stars associated with Orion A. This sample of variable stars was analyzed as an ensemble to establish the characteristics of the amplitude, timescales, and any correlated magnitudecolor fluctuations for pre-main-sequence stars. The mean peak-to-peak photometric fluctuation is $\sim 0.2 \mathrm{mag}$ in each band, although the more extreme variable stars have amplitudes as large as $\sim 2 \mathrm{mag}$. Most of the variability is essentially colorless within the photometric noise, with $77 \%$ of the variable stars having peak-to-peak $J-H$ color variations less than $0.05 \mathrm{mag}$. The timescales of the photometric fluctuations as established from the autocorrelation function are typically less than a few days. Only $\sim 2 \%$ of the variable stars were identified based solely on their longterm variability with amplitude fluctuations $\gtrsim 0.1 \mathrm{mag}$, suggesting that most near-infrared variability is a result of short-term processes.

The variability characteristics established from these observations constrain the mechanisms that lead to nearinfrared photometric variability in individual sources. While any variety of mechanism can produce the observed low-amplitude fluctuations, stars with low amplitudes typically show colorless photometric variations and nearinfrared colors consistent with reddened photospheres (i.e., 
lack near-infrared excesses), and often they exhibit periodic photometric fluctuations. These characteristics are analogous to those observed in type I optical variability in the Herbst et al. (1994) classification scheme, and we suggest that the variability in $56 \%-77 \%$ of the stars is a result of rotational modulation by cool spots. Cool spots cannot explain the $23 \%$ of the stars that have color variations $\gtrsim 0.05 \mathrm{mag}$, however, and alternate mechanisms were investigated to explain these variability characteristics, including hot spots, extinction, and accretion disks. Rotational modulation by hot spots and extinction variations can account for the amplitude of the photometric variations and the observed correlation between the $J$ and $J-H$ variations. However, the more extreme variable stars would require spot temperatures and spot coverages larger than inferred from optical observations. In addition, neither hot spots nor extinction can quantitatively explain the correlations between the magnitude and color variations in the $J-H$ versus $H-K_{s}$ and $K_{s}$ versus $H-K_{s}$ diagrams or account for stars that become bluer as they fade. Accretion disk models were investigated as another source of nearinfrared variability. By invoking changes in the mass accretion rate or changes in the inner hole radius of the circumstellar disk (whether by dynamical, opacity, or temperature variations), accretion disk models can account for the $\sim 17$ stars that become bluer as they decrease in brightness. Like hot spots and extinction, however, accretion disk models cannot explain the detailed distribution of stars in the color-color diagram. This suggests either that another variability mechanism not considered here may be oper- ative or that the observed variability represents the net results of several of these phenomena. In particular, hot spots, extinction variations, and accretion variations all relate to the properties of the circumstellar disk, and it is plausible, if not likely, that more than one of these mechanisms is simultaneously contributing to the near-infrared variability in many of these stars.

We thank Nuria Calvet for allowing us to use the accretion disk model results shown in Figure 25, Bill Herbst for discussions about photometric periodicity in young stellar objects, and both Bill Herbst and Luisa Rebull for sharing optical periodicity results with us prior to publication. We would also like to thank the 2MASS observatory staff and data management team for acquiring and pipelineprocessing the special survey observations used in this investigation. This publication makes use of data products from the Two Micron All Sky Survey, which is a joint project of the University of Massachusetts and the Infrared Processing and Analysis Center, funded by the National Aeronautics and Space Administration and the National Science Foundation. The 2MASS science data and information services were provided by the Infrared Science Archive (IRSA) at IPAC. This research has made use of the SIMBAD database, operated at CDS, Strasbourg, France. J. M. C. acknowledges support from Long-Term Space Astrophysics grant NAG 5-8217 and the Owens Valley Radio Observatory, which is supported by the National Science Foundation through grant AST 99-81546.

\section{APPENDIX}

\section{THE STELLAR POPULATION IN THE ORION A MOLECULAR CLOUD}

Hillenbrand \& Hartmann (1998) demonstrated that the Trapezium cluster, a $\sim 3^{\prime} \times 5^{\prime}$ region centered near $\theta^{1}$ Ori $\mathrm{C}$ (Herbig \& Terndrup 1986), constitutes the core of the Orion Nebula cluster. They further showed that the Orion Nebula cluster contains upward of a few thousand stars extended over at least an $18^{\prime}(2.5 \mathrm{pc})$ region and is elongated along the direction of the Orion A molecular cloud with an aspect ratio of $\sim 2: 1$. Ali \& DePoy (1995) indicated, though, that the observed $K$-band stellar surface density is higher than the expected field star density over much of the $39^{\prime} \times 39^{\prime}$ region they surveyed. Carpenter (2000) further found from analysis of the 2MASS Second Incremental Release data that the enhanced $K_{s}$-band star counts extend for $\sim 2^{\circ}$ in declination. The observations obtained here are spatially more complete than the data analyzed in that study and allow for a more thorough investigation of the spatial extent and membership of the northern portion of the Orion A molecular cloud, as now described.

In general, it is not possible to identify the individual stars associated with the Orion A molecular cloud from $J$, $H$, and $K_{s}$ photometry alone unless a star has a nearinfrared excess. Since not all stars have such distinctive colors, we identified the spatial distribution of stars statistically by subtracting the expected field star population from the observed star counts using the semiempirical procedure described by Carpenter (2000). Briefly, the field star contamination in the absence of extinction from the Orion A molecular cloud itself was assessed using the regions $\left(\alpha<84^{\circ}, \delta<-7^{\circ}\right)$ and $\delta>-4^{\circ}$, which are off of the molec- ular cloud and away from the stellar density peaks (see Fig. 14). The mean $K_{s}$-band surface density in these regions is $0.66 \mathrm{arcmin}^{-2}$ for stars brighter than $K_{s}=14.8 \mathrm{mag}$ and $0.006 \mathrm{arcmin}^{-2}$ for the variable stars. These results indicate that the field star contamination of the variable population is only $\sim 7 \%$, and it is therefore neglected throughout this paper. The field star surface density for the $K_{s} \leq 14.8$ star counts decreases by $\sim 10 \%$ from $\delta>-4.2$ to $\delta<-7^{\circ}$, which is consistent with the expected variation predicted by Galactic star-count models due to variations in the Galactic latitude across the region surveyed (Wainscoat et al. 1992). The variation with Galactic longitude is predicted to be $\sim 1 \%$. The expected surface density of unreddened field stars was estimated by making a linear fit to the stellar surface density map in the off-cloud regions as a function of Galactic latitude. Extinction from the Orion A molecular cloud, however, will decrease the number of background field stars predicted by this linear fit. The reddened field star density as a function of position in the cloud was estimated using the ${ }^{13} \mathrm{CO}(1-0)$ map from Bally et al. (1987) as a tracer of the cloud extinction and the Wainscoat et al. (1992) starcount model to estimate the fraction of field stars that are background to the molecular cloud (see Carpenter 2000 for complete details). This reddened field star model was subtracted from the observed surface density map to yield the stellar population associated with the Orion A molecular cloud.

Generally, the star-count analysis should be performed over all $K_{s}$-band magnitudes to obtain the most complete 


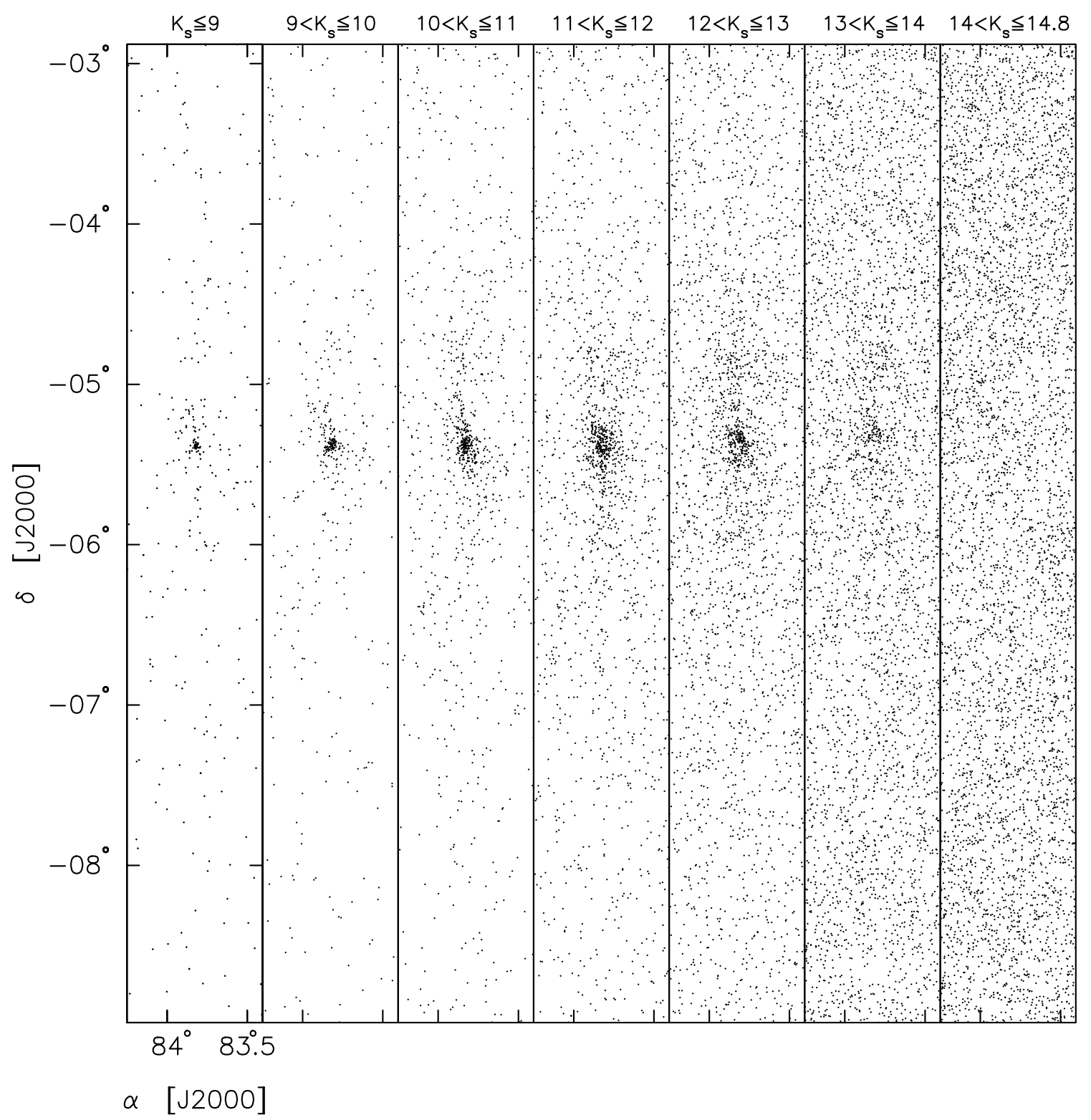

FIG. 27.- Spatial distribution of stars in differential $K_{s}$ magnitude intervals. This figure demonstrates that the stellar population associated with the Orion A molecular cloud is prominent only for $K_{s} \lesssim 14$. At fainter magnitudes, the star counts are dominated by the field star population.

estimate of the stellar population. In practice, a spatial enhancement in the stellar surface density is only prominent at brighter magnitudes, as can be seen in Figure 27, which shows the spatial distribution of stars in differential magnitude intervals. An excess of stars is discerned against the field star population for magnitudes $K_{s} \lesssim 13$, but it is no longer prominent at $K_{s} \gtrsim 14$ (see also Fig. 15). Including the star counts fainter than $K_{s}=14$ will add noise to the statistical analysis, and therefore only stars with magnitudes $K_{s} \leq 14$ were used in this analysis.

The extent and number of stars in the Orion A molecular cloud were estimated by forming closed contours in the field star-subtracted surface density map at various density levels. For each density, we computed the number of observed stars in excess of the field star model, the number of variable stars, and the spatial extent, assuming a distance of $480 \mathrm{pc}$ (Genzel et al. 1981). The spatial extent is characterized by a major and a minor axis, defined as the largest angular extent in declination at the defined surface density level and the perpendicular angular dimension needed in order to reproduce the total contour area, respectively. The lowest surface density considered begins at $\sim 1 \sigma(0.25$ $\operatorname{arcmin}^{-2}$ ) above the mean field star density, where $\sigma$ is the Poisson noise in the field star contamination in the absence of extinction from the molecular cloud. Table 5 summarizes the angular extent and number of stars as a function of the surface density. At the lowest surface density level identified here, the closed contour extends over a $0.4 \times 2.4$ region $(3.4 \mathrm{pc} \times 20 \mathrm{pc})$ and contains $\sim 2700$ stars brighter than $K_{s}=14$. These source counts represent a lower limit to the total stellar population in the northern portion of the Orion A molecular cloud due to unresolved binaries and the difficulty in identifying point sources in the Trapezium region, with its bright nebular background and high overall source density. A more global star-count analysis indicates that these observations cover the full area of the stellar density enhancement even though the Orion A molecular cloud extends further to the southeast (Carpenter 2000). The 
angular extent of the density enhancement is similar to that seen in OB stars and $\mathrm{H} \alpha$-emitting objects (Gomez \& Lada 1998).

The lowest surface density contour encompasses several regions that have been previously labeled as individual clusters, including the ONC, the Trapezium (which is now considered part of the ONC; Hillenbrand \& Hartmann 1998), L1641 N (Strom, Strom, \& Merrill 1993; Hodapp \& Deane 1993), OMC-2 (Jones et al. 1994), smaller groups of stars associated with low-luminosity IRAS sources (Chen \& Tokunaga 1994), and a more dispersed grouping of stars near NGC 1977. While each of these regions represents a local stellar density peak, the results obtained here suggest that they are all connected as part of an extended ridge of stars that have formed within a localized region of the Orion A molecular cloud. This assertion is supported by the spatial continuity of the enhanced star counts, their close correspondence to the shape of Orion A molecular cloud (see Fig. 14), and observational signatures of young stellar ages $(\sim 1 \mathrm{Myr})$ over the entire region, either from direct spectroscopic evidence (Hodapp \& Deane 1993; Hillenbrand 1997) or from the presence of outflows and nearinfrared excesses common in pre-main-sequence stars
(Strom et al. 1993; Chen \& Tokunaga 1994; Jones et al. 1994).

Whether the enhanced ridge of star counts should be identified as a single object or several clusters is somewhat ambiguous. Historically, clusters have been defined as gravitationally bound groups of stars. The elongated shape of the stellar density enhancement observed in Orion A suggests that it is unlikely it will persist as a bound group once the molecular gas is dispersed. Localized regions within the stellar density enhancement may remain gravitationally bound, though, and specifically, the ONC as defined by Hillenbrand \& Hartmann (1998) may emerge as a cluster if a factor of $\sim 2$ more stars are formed in the $2.5 \mathrm{pc}$ region centered on the Trapezium. Near-infrared star-count analyses in the past decade, though, have loosely identified clusters as groups of stars forming within a localized region of a molecular cloud that may not necessarily emerge as gravitationally bound entities. By this definition, the boundaries of the previously identified ONC, L1641 N, and OMC-2 clusters are not easily distinguished, and it is not clear how, or if, the stellar density enhancement should be divided into separate clusters.

\section{REFERENCES}

Ali, B., \& DePoy, D. L. 1995, AJ, 109, 709

Antokhina, É. A., Ismailov, N. Z., \& Cherepashchuk, A. M. 1989, AZh

Pisma, 15, 837 (English transl. Soviet Astron. Lett., 15, 362)

Armitage, P. J. 1995, MNRAS, 274, 1242

Aspin, C., \& Sandell, G. 1994, A\&A, 288, 803

Bally, J., Stark, A. A., Wilson, R. W., \& Langer, W. D. 1987, ApJ, 312, L45

Basri, G., Johns-Krull, C. M., \& Mathieu, R. D. 1997, AJ, 114, 781

Bertout, C. 2000, A\&A, 363, 984

Bertout, C., Harder, S., Malbet, F., Mennessier, C., \& Regev, O. 1996, AJ, 112,2159

Bessell, M. S., \& Brett, J. M. 1988, PASP, 100, 1134

Bevington, P. R. 1969, Data Reduction and Error Analysis for the Physical Sciences (New York: McGraw-Hill)

Bouvier, J., \& Bertout, C. 1989, A\&A, 211, 99

Bouvier, J., Bertout, C., Benz, W., \& Mayor, M. 1986, A\&A, 165, 110

Bouvier, J., Cabrit, S., Fernández, M., Martín, E. L., \& Matthews, J. M. 1993, A\&A, 272, 176

Bouvier, J., et al. 1999, A\&A, 349, 619

Bouvier, J., Covino, E., Kovo, O., Martín, E. L., Matthews, J. M. Terranegra, L., \& Beck, S. C. 1995, A\&A, 299, 89

Brown, A. G. A., de Geus, E. J., \& de Zeeuw, P. T. 1994, A\&A, 289, 101

Carpenter, J. M. 2000, AJ, 120, 3139

2001, AJ, 121, 2851

Chen, H., \& Tokunaga, A. T. 1994, ApJS, 90, 149

Clarke, C. J., Armitage, P. J., Smith, K. W., \& Pringle, J. E. 1995, MNRAS, 273,639

Cohen, J. G., Frogel, J. A., Persson, S. E., \& Elias, J. H. 1981, ApJ, 249, 481

Cohen, M., \& Schwartz, R. D. 1976, MNRAS, 174, 137

Comerón, F., Rieke, G. H., \& Neuhäuser, R. 1999, A\&A, 343, 477

Cutri, R. M. et al. 2000, Explanatory Supplement to the 2MASS Second Incremental Data Release (Pasadena: Infrared Processing Anal. Cent.)

D'Antona, F., \& Mazzitelli, I. 1997, Mem. Soc. Astron. Italiana, 68, 807

Fernández, M., \& Eiroa, C. 1996, A\&A, 310, 143

Frank, J., King, A., \& Raine, D. 1985, Accretion Power in Astrophysics (2d ed.; Cambridge: Cambridge Univ. Press)

Genzel, R., Reid, M. J., Moran, J. M., \& Downes, D. 1981, ApJ, 244, 884

Gomez, M., \& Lada, C. J. 1998, AJ, 115, 1524 (erratum 116, 1508)

Greene, T. P., \& Meyer, M. R. 1995, ApJ, 450, 233

Grinin, V. P., Rostopchina, A. N., \& Shakhovskoi, D. N. 1998, AZh Pisma, 24, 925 (English transl. Astron. Lett., 24, 802)

Guenther, E. W., et al. 2000, A\&A, 357, 206

Gullbring, E., Petrov, P. P., Ilyin, I., Tuominen, I., Gahm, G. F., \& Lodén, K. 1996, A\&A, 314, 835

Harder, S., Bertout, C., \& Mennessier, C. 1998, A\&AS, 129, 337

Hartmann, L. 2001, AJ, 121, 1030

Hartmann, L., Calvet, N., Gullbring, E., \& D'Alessio, P. 1998, ApJ, 495, 385

Hartmann, L., Hewett, R., \& Calvet, N. 1994, ApJ, 426, 669

Hartmann, L., Hewett, R., Stahler, S., \& Mathieu, R. D. 1986, ApJ, 309, 275

Hartmann, L., Kenyon, S., \& Hartigan, P. 1993, in Protostars and Planets

III, ed. E. H. Levy \& J. I. Lunine (Tucson: Univ. Arizona Press), 497

Herbig, G. H. 1962, Adv. Astron. Astrophys., 1, 47

-. 1977, ApJ, 217, 693
Herbig, G. H., \& Terndrup, D. M. 1986, ApJ, 307, 609

Herbst, W., Herbst, D. K., Grossman, E. J., \& Weinstein, D. 1994, AJ, 108, 1906

Herbst, W., Maley, J. A., \& Williams, E. C. 2000a, AJ, 120, 349

Herbst, W., Rhode, K. L., Hillenbrand, L. A., \& Curran, G. 2000b, AJ, 119, 261

Herbst, W., \& Shevchenko, V. S. 1999, AJ, 118, 1043

Hillenbrand, L. A. 1997, AJ, 113, 1733

Hillenbrand, L. A., \& Carpenter, J. M. 2000, ApJ, 540, 236

Hillenbrand, L. A., Carpenter, J. M., \& Skrutskie, M. F. 2001, ApJ, 547, L53

Hillenbrand, L. A., \& Hartmann, L. W. 1998, ApJ, 492, 540

Hillenbrand, L. A., Strom, S. E., Calvet, N., Merrill, K., M., Gatley, I., Makidon, R. B., Meyer, M. R., \& Skrutskie, M. F. 1998, AJ, 116, 1816

Hodapp, K.-W. 1999, AJ, 118, 1338

Hodapp, K.-W., \& Deane, J. 1993, ApJS, 88, 119

Horrobin, M. J., Casali, M. M., \& Eiroa, C. 1997, A\&A, 320, L41

Hutchinson, M. G., Evans, A., Davies, J. K., \& Bode, M. F. 1989, MNRAS, 237,683

Johns-Krull, C. M., \& Hatzes, A. P. 1997, ApJ, 487, 896

Joncour, I., Bertout, C., \& Bouvier, J. 1994, A\&A, 291, L19

Jones, T. J., Mergen, J., Odewahn, S., Gehrz, R. D., Gatley, I., Merrill, K. M., Probst, R., \& Woodward, C. E. 1994, AJ, 107, 2120

Joy, A. H. 1945, ApJ, 102, 168

Kaas, A. A. 1999, AJ, 118, 558

Kenyon, S. J., et al. 1994, AJ, 107, 2153

Kenyon, S. J., Yi, I., \& Hartmann, L. 1996, ApJ, 462, 439

Königl, A. 1991, ApJ, 370, L39

Lada, C. J., \& Adams, F. C. 1992, ApJ, 393, 278

Lada, C. J., Alves, J., \& Lada, E. A. 1996, AJ, 111, 1964

Lai, D. 1999, ApJ, 524, 1030

Liseau, R., Lorenzetti, D., \& Molinari, S. 1992, A\&A, 253, 119

Luhman, K. L., Rieke, G. H., Lada, C. J., \& Lada, E. A. 1998, ApJ, 508, 347

Lynden-Bell, D., \& Pringle, J. E. 1974, MNRAS, 168, 603

Mahdavi, A., \& Kenyon, S. J. 1998, ApJ, 497, 342

McCaughrean, M. J., Zinnecker, H., Rayner, J. T., \& Stauffer, J. R. 1995, in The Bottom of the Main Sequence-And Beyond, ed. C. G. Tinney (Berlin: Springer), 209

Ménard, F., \& Bertout, C. 1999, in The Origin of Stars and Planetary Systems, ed. C. J. Lada \& N. D. Kylafis (Dordrecht: Kluwer), 341

Meyer, M. R. 1996, Ph.D. thesis, Univ. Massachusetts

Meyer, M. R., Calvet, N., \& Hillenbrand, L. A. 1997, AJ, 114, 288

Muench, A. A., Lada, E. A., \& Lada, C. J. 2000, ApJ, 533, 358

Neuhäuser, R., et al. 1998, A\&A, 334, 873

Parsamian, E. S., \& Chavira, E. 1982, Bol. Inst. Tonantzintla, 3, 69

Press, W. H., Teukolsky, S. A., Vetterling, W. T., \& Flannery, B. P. 1992,

Numerical Recipes in C (2d ed.; Cambridge: Cambridge Univ. Press) Rebull, L. M. 2001, AJ, 121, 1676

Rice, J. B., \& Strassmeier, K. G. 1996, A\&A, 316, 164

Rydgren, A. E., \& Vrba, F. J. 1983, AJ, 88, 1017

Scargle, J. D. 1989, ApJ, 343, 874

Shakura, N. I., \& Sunyaev, R. A. 1973, A\&A, 24, 337

Simon, M., Close, L. M., \& Beck, T. L. 1999, AJ, 117, 1375 
Skrutskie, M. F., Meyer, M. R., Whalen, D., \& Hamilton, C. 1996, AJ, 112, 2168

Smith, K. W., Bonnell, I. A., \& Lewis, G. F. 1995, MNRAS, 276, 263

Smith, K. W., Lewis, G. F., Bonnell, I. A., Bunclark, P. S., \& Emerson, J. P. 1999, MNRAS, 304, 367

Stassun, K. G., Mathieu, R. D., Mazeh, T., \& Vrba, F. 1999, AJ, 117, 2941

Stetson, P. B. 1987, PASP, 99, 191

1996, PASP, 108, 851

Strom, K. M., Strom, S. E., \& Merrill, K. M. 1993, ApJ, 412, 233

Terquem, C., \& Papaloizou, J. C. B. 2000, A\&A, 360, 1031

Vrba, F. J., Chugainov, P. F., Weaver, W. B., \& Stauffer, J. S. 1993, AJ, 106, 1608

Vrba, F. J., Rydgren, A. E., Chugainov, P. F., Shakovskaya, N. I., \& Weaver, W. B. 1989, AJ, 97, 483

Vrba, F. J., Rydgren, A. E., Chugainov, P. F., Shakovskaya, N. I., \& Zak, D. S. 1986, ApJ, 306, 199
Wainscoat, R. J., Cohen, M., Volk, K., Walker, H. J., \& Schwartz, D. E. 1992, ApJS, 83, 111

Walker, M. F. 1972, ApJ, 175, 89

. 1983, ApJ, 271, 642

Welch, D. L. \& Stetson, P. B. 1993, AJ, 105, 1813

Wiramihardja, S. D., Kogure, T., Yoshida, S., Nakano, M., Ogura, K., \& Iwata, T. 1991, PASJ, 43, 27

Wiramihardja, S. D., Kogure, T., Yoshida, S., Ogura, K., \& Nakano, M. 1993, PASJ, 45, 643

Yudin, R. V. 2000, A\&AS, 144, 285

Zinnecker, H., McCaughrean, M. J., \& Wilking, B. A. 1993, in Protostars and Planets III, ed. E. H. Levy \& J. I. Lunine (Tucson: Univ. Arizona Press), 429 